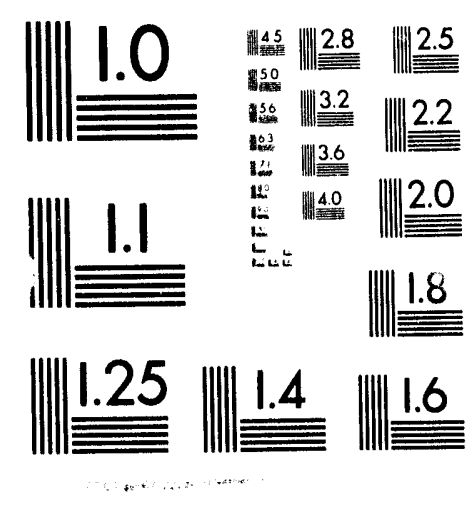



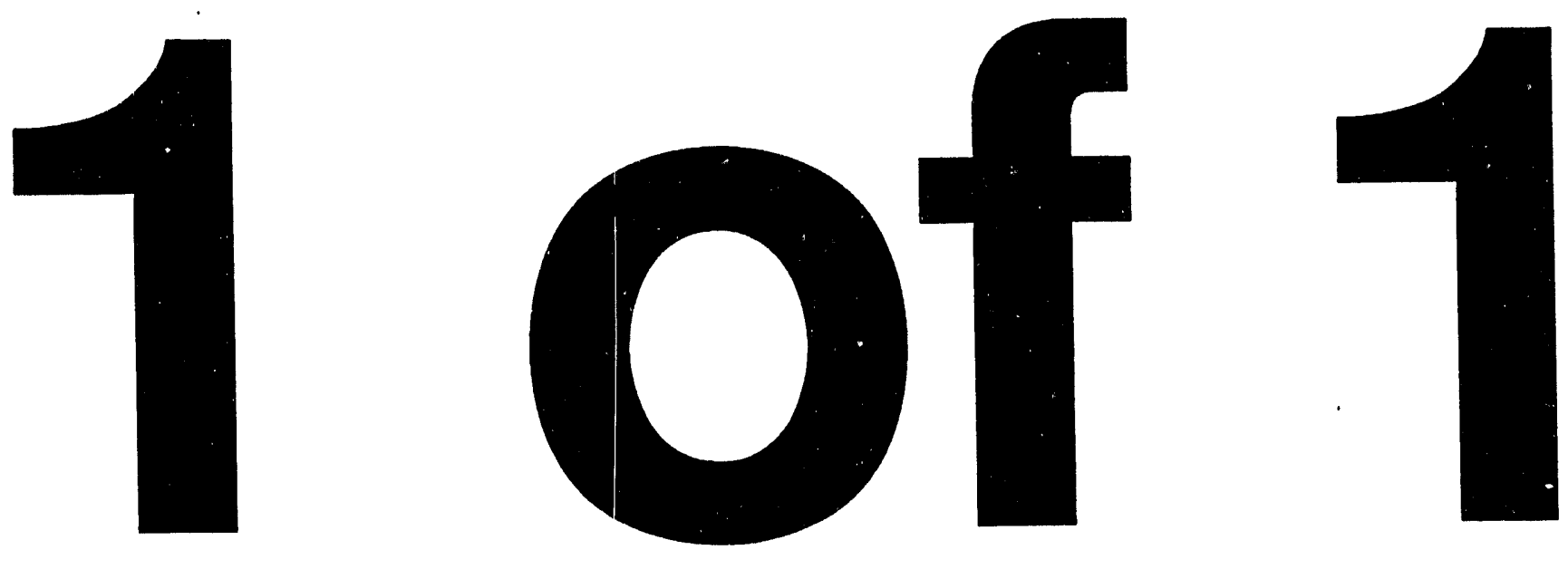
LBL-34784

UC-414

\title{
Measurement of Tau Lepton Branching Fractions
}

\author{
Neil Allen Nicol \\ Ph.D. Thesis \\ Department of Physics \\ University of California, Berkeley, CA 94720 \\ and \\ Lawrence Berkeley Laboratory \\ University of California, Berkeley, CA 94720
}

September 30, 1993

\begin{abstract}
We present $\tau^{-}$lepton branching fraction measurements based on data from the TPC/Two-Gamma detector at PEP. Using a sample of $\tau^{-} \rightarrow \nu_{\tau} K^{-} \pi^{+} \pi^{-}$events, we examine the resonance structure of the $K^{-} \pi^{+} \pi^{-}$system and obtain the first measurements of branching fractions for $\tau^{-} \rightarrow \nu_{\tau} K_{1}^{-}(1270)$ and $\tau^{-} \rightarrow \nu_{\tau} K_{1}^{-}(1400)$. We also describe a complete set of branching fraction measurements in which all the decays of the $\tau^{-}$lepton are separated into classes defined by the identities of the charged particles and an estimate of the number of neutrals. This is the first such global measurement with decay classes defined by the four possible charged particle species, $e, \mu, \pi$, and $K$.
\end{abstract}

This work was supported by the Director, Office of Energy Research, Office of High Energy and Nuclear Physics, Division of High Energy Physics, of the U.S.

Department of Energy under contract DE-AC03-76SF00098. 
FOR Lynda, Kip, Scot, Tad 


\section{Acknowledgements}

At the time that I became a member of the TPC/Two-Gamma collaboration it was already a mature experiment with a considerable history and folklore. I have been fortunate to work with this community of physicists, engineers, and technicians, and to learn from their experience. I am grateful to each of them for their efforts in building, operating, and understanding the TPC/Two-Gamma experiment.

My advisor, Mike Ronan, has provided steady guidance in all aspects of the experiment throughout my tenure as a graduate student researcher, and I thank him for his encouragement and support. I am also indebted to Gerry Lynch, Ron Ross, Philippe Eberhard, Jeremy Lys, Al Clark, Orin Dahl, Al Eisner, Lynn Stevenson, David Lambert and Mahiko Suzuki for their willingness to answer all my questions. Their doors were always open and their suggestions sound.

Finally, my thanks go out to the members of my dissertation committee, Mike Ronan, Ron Ross, Harry Bingham, and Selig Kaplan, for their thorough reading and useful comments. 


\section{Contents}

$\begin{array}{ll}\text { Dedication } & \text { ii }\end{array}$

Acknowledgements $\quad$ iii

1 Overview 1

2 Physics of Tau Lepton Decays 3

2.1 Particles and Interactions ................. 3

2.2 The Tau Lepton . . . . . . . . . . . . . . . . . . 4 4

2.3 Decays of the Tau Lepton . . . . . . . . . . . . . . . 4

2.4 Leptonic Decays . . . . . . . . . . . . . . . . . . . . 6

2.5 Hadronic Decays . . . . . . . . . . . . . . . . . 8

2.5.1 Resonances ................. 8

2.5 .2 Final States . . . . . . . . . . . . . . 10

2.5.3 Quark Model of Mesons ... . . . . . . . . . 12

2.5.4 Photons in Tau Decays . . . . . . . . . . . . . 14

2.6 Discussion ......................... 15

3 The TPC/Two-Gamma Experiment 16

3.1 SLAC and the PEP Collider . . . . . . . . . . . . . . . 16

3.2 Physics at PEP . . . . . . . . . . . . . . 17

3.3 The TPC/Two-Gamma Detector . . . . . . . . . . . . . . 19

3.3.1 Time Projection Chamber . . . . . . . . . . . 19

3.3.2 Electromagnetic Calorimeters . . . . . . . . . . . 23

3.3.3 Muon Chambers .................. 25

3.4 Data Acquisition and Analysis . . . . . . . . . . . . 26

3.4 .1 Trigger ................... 27 
3.4.2 Event Reconstruction . . . . . . . . . . . . . . 27

3.4 .3 Integrated Luminosity . . . . . . . . . . . . . . . 28

4 Measurement of $\tau^{-} \rightarrow \nu_{\tau} K_{1}^{-}$Branching Fractions 29

4.1 The Method . . . . . . . . . . . . . . . . . . . 29

4.2 Event Selection . . . . . . . . . . . . . . . 32

4.3 Charged Particle Identification . . . . . . . . . . . . . . 33

4.4 Neutral Particle Identification . . . . . . . . . . . . . . 37

4.5 Decay Models . . . . . . . . . . . . . . . . . 38

4.6 Branching Fractions . . . . . . . . . . . . . . . . . 42

4.7 Discussion . . . . . . . . . . . . . . . . . 45

5 Global Measurement of $\tau^{-}$Branching Fractions 46

5.1 The Method . . . . . . . . . . . . . . . . . 47

5.2 Event Selection . . . . . . . . . . . . . . . . 52

5.2 .1 Selection Criteria . . . . . . . . . . . . . 52

5.2 .2 Kinematic Selection . . . . . . . . . . . . . 53

5.2 .3 Particle Identification Selection . . . . . . . . . . 56

5.2 .4 Selection Efficiencies ............... 60

5.3 Event Classification . . . . . . . . . . . . . . . . 61

5.3.1 Charged Tracks ... . . . . . . . . . . . 62

5.3 .2 Neutrals . . . . . . . . . . . . . . . . . . 62

5.3.3 Event Class Populations . . . . . . . . . . . . . . 64

5.3.4 Classification Efficiencies ............. 66

5.4 Branching Fractions . . . . . . . . . . . . . . . 68

5.4.1 Estimation of Systematic Errors . . . . . . . . . . . 69

5.4 .2 Decay Classes . . . . . . . . . . . . . . 71

5.4 .3 Resonance Classes ................ . 73

5.5 Discussion .................... . . 75

6 Conclusions $\quad 76$

$\begin{array}{ll}\text { Bibliography } & \mathbf{7 8}\end{array}$ 


\section{List of Tables}

$2.1 \tau^{-}$decays and branching fractions $\ldots \ldots \ldots \ldots \ldots \ldots \ldots$

$2.2 q \bar{q}$ flavor and $J^{P C}$ states $\ldots \ldots \ldots \ldots \ldots \ldots \ldots$

3.1 Total event estimates for the TPC/Two-Gamma experiment $\ldots 28$

4.1 Properties of the $K_{1}^{-}$resonances $\ldots \ldots \ldots \ldots \ldots$

4.2 Decay modes of the $K_{1}^{-}$resonances $\ldots \ldots \ldots \ldots \ldots$

4.3 Selection efficiencies for $\tau_{1+3}$ events $\ldots \ldots \ldots \ldots \ldots \ldots$

4.4 Three-prong decay class populations $\ldots \ldots \ldots \ldots \ldots \ldots$

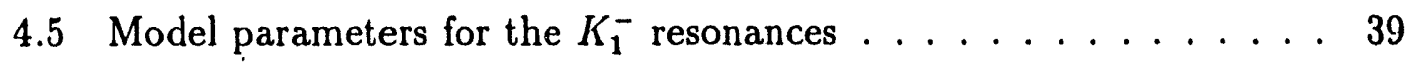

4.6 Normalized decay fractions for $K_{1}^{-} \rightarrow K^{-} \pi^{+} \pi^{-} \ldots \ldots \ldots \ldots$. . . 41

4.7 Branching fractions for $\tau^{-} \rightarrow \nu_{\tau} K_{1}^{-}(1270)$ and $\tau^{-} \rightarrow \nu_{\tau} K_{1}^{-}(1400) \ldots 41$

$4.8 \tau^{-}$branching fractions for $\bar{u} d$ and $\bar{u} s$ resonant states $\ldots \ldots \ldots 45$

$5.1 \tau^{-}$decay classes $\ldots \ldots \ldots \ldots \ldots \ldots \ldots \ldots \ldots$

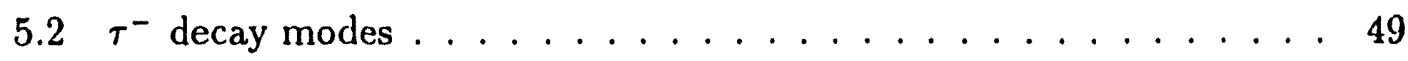

5.3 Charged track identification for selection . . . . . . . . . 58

5.4 Event selection efficiencies for dominant channels . . . . . . . . 60

5.5 Event selection efficiencies for additional $\bar{u} s$ channels . . . . . . 61

5.6 Charged track identification for classification . . . . . . . . 63

5.7 Distributions of reconstructed $\pi^{0}$ and additional photons . . . . 66

5.8 Decay class populations . . . . . . . . . . . . . 67

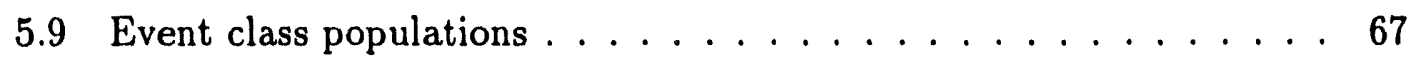

5.10 Classification efficiencies of $\tau^{+} \rightarrow \bar{\nu}_{\tau} \pi^{+} \pi^{0} / \tau^{-} \rightarrow \nu_{\tau} \bar{\nu}_{e} e^{-}$decays $\ldots 68$

5.11 Sytematic errors for decay class branching fractions . . . . . 70

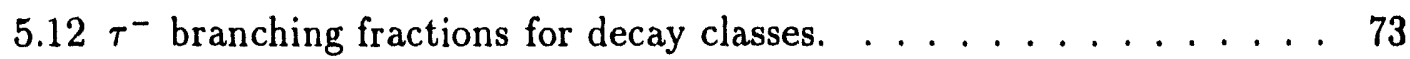

$5.13 \tau^{-}$branching fractions for resonant states $\ldots \ldots \ldots \ldots 74$ 


\section{List of Figures}

2.1 Generations of leptons and quarks . . . . . . . . . . . . 3

2.2 Particle doublets at the $W^{-}$vertex in $\tau^{-}$decays . . . . . . . . 5

2.3 Spectroscopy of $\bar{u} d$ quark states . . . . . . . . . . . . . . . 9

2.4 Spectroscopy of $\bar{u} s$ quark states . . . . . . . . . . . . . . 10

3.1 SLAC linear accelerator and PEP collider . . . . . . . . . . . . 17

$3.2 \mathrm{TPC} /$ Two-Gamma detector cross-sectional side view . . . . . . . . 20

3.3 TPC/Two-Gamma detector cross-sectional end view. . . . . . . . 21

3.4 Time projection chamber sector . . . . . . . . . . . . 22

$3.5 \mathrm{~d} E / \mathrm{d} x$ vs momentum for the charged tracks of a $\tau^{+} \tau^{-}$event sample 23

3.6 Calorimeter laminate and wire mesh . . . . . . . . . . . . . 24

3.7 Calorimeter energy vs momentum for a sample of $\tau^{+} \tau^{-}$events . . . 25

3.8 Muon chamber array . . . . . . . . . . . . . . . . 26

$4.1 \mathrm{~d} E / \mathrm{d} x$ vs momentum for three-prong tracks .......... 34

$4.2 \chi_{K}$ vs $\chi_{\pi}$ for three-prong tracks ................ 35

4.3 Mass distributions of the $\mathrm{K}^{-} \pi^{+} \pi^{-}$candidate events . . . . . . . . 37

4.4 Mass distributions of the $K_{1}^{-}(1270)$ and $K_{1}^{-}(1400)$ models . . . . . 42

4.5 Mass distributions of best fit $K_{1}^{-}(1270)$ and $K_{1}^{-}(1400) \quad \ldots . \ldots 43$

4.6 Statistical error contours for the $\tau^{-} \rightarrow \nu_{\tau} K_{1}^{-}$branching fractions . . 44

5.1 Overview of analysis .................... 51

5.2 Energy/momentum function . . . . . . . . . . . . . . 57

$5.3 \mathrm{~d} E / \mathrm{d} x$ distributions for event selection $\ldots \ldots \ldots \ldots$

5.4 Angular distribution of photons .............. . . 64

5.5 Invariant mass of photon pairs . . . . . . . . . . . 65

5.6 Mass distributions for $\pi^{-} \pi^{0}, K^{-} \pi^{0}$, and $\pi^{-} \pi^{+} \pi^{-}$events . . . . . 72 


\section{Chapter 1}

\section{Overview}

Measurements of the branching fractions of $\tau^{-}$lepton decays provide a number of important checks of the standard model of particle physics. Through the interaction of the $W^{-}$, the $\tau^{-}$couples to $\nu_{\tau}$ and a pair of leptons or quarks. The rates for the leptonic decays, $\tau^{-} \rightarrow \nu_{\tau} \ddot{\nu}_{e} e^{-}$and $\tau^{-} \rightarrow \nu_{\tau} \bar{\nu}_{\mu} \mu^{-}$, are predicted very precisely from electroweak theory. Measurements of the $\tau^{-}$lifetime and leptonic branching fractions provide an important check of these predicted rates. Measurements of the hadronic decays give information concerning the coupling of the weak hadronic currents. Since the $\nu_{\tau}$ interacts only weakly, the hadronic decays of the $\tau^{-}$produce an isolated quark system, either $\bar{u} d$ or $\bar{u} s$, which is ideal for studies of the strong interaction below the $\tau^{-}$mass.

The analysis described in this thesis is based on data from the TPC/TwoGamma detector, which has excellent overall charged particle identification and is nearly unique in its ability to separate charged pions, kaons, and protons. This feature allows us to examine the $\tau^{-}$decay modes which contain $K^{ \pm}$. Although these modes have very small branching fractions, the physical insight they provide is comparable to that of modes without $K^{ \pm}$, since the ability to detect $K^{ \pm}$gives a signature for the reconstruction of the $\bar{u} s$ decays of the $\tau^{-}$. This thesis includes the first measurement of the branching fractions for the decays $\tau^{-} \rightarrow \nu_{\tau} K_{1}^{-}(1270)$ and $\tau^{-} \rightarrow \nu_{\tau} K_{1}^{-}(1400)$. Using the TPC detector, we are able to select a pure sample of $\tau^{-} \rightarrow \nu_{\tau} K^{-} \pi^{+} \pi^{-}$events which we use for this resonance analysis.

For many years there have been questions concerning the inability of experiments to arrive at a consistent set of branching fractions for the decays of the $\tau^{-}$ lepton. The sum of branching fraction measurements for exclusive decay modes falls significantly short of unity $[1,2]$. The outstanding question is whether there are unobserved decay modes that have not been included in the existing measurements or if the current measurements are systematically low. One approach to resolve this dilemma is to devise a set of selection criteria that would include all of the possible decay modes of $\tau^{+} \tau^{-}$events. The decays in the sample are then classified into a broad set of categories to allow the simultaneous measurement of 
all modes. This method serves as a check that low values for certain decay modes are not accompanied by high measurements somewhere else.

The final analysis described in this thesis is a global measurement in which all of the decays of the $\tau^{-}$are separated into a number of decay classes. Although this approach was used originally by CELLO [3], and more recently by ALEPH [4], the TPC/Two-Gamma analysis described here is the first which distinguishes charged pions and kaons. This particle identification capability allows us to use a much finer definition of decay classes which separates the hadronic decays into those originating from $\bar{u} d$ and $\bar{u} s$ quark systems.

We begin the bulk of the thesis with a chapter on the theoretical context for our measurements of $\tau^{-}$decays. We review the current status of theoretical and experimental values for the $\tau^{-}$branching fractions and discuss the contributions of this analysis, with emphasis on the observation and reconstruction of decays with $K^{ \pm}$. The next chapter provides an experimental context, essentially a brief description of the TPC/Two-Gamma detector used to observe and record the data upon which the analysis is based. With the theoretical and experimental foundation in place, we proceed with two chapters that detail the data analysis leading to our branching fraction measurements for $\tau^{-} \rightarrow \nu_{\tau} K_{1}^{-}$and then for a comprehensive set of $\tau^{-}$decay classes. We conclude with a summary of our results and a discussion of their significance.

Throughout this thesis we use the notation of $\tau^{-}$decays to implicitly include both $\tau^{-}$and charge conjugate $\tau^{+}$processes. 


\section{Chapter 2}

\section{Physics of Tau Lepton Decays}

The decays of $\tau^{+} \tau^{-}$lepton pairs produced at $e^{+} e^{-}$colliders provide an exceptionally clean environment for studies of weak charged coupling and strong interactions below the $\tau^{-}$mass. The leptonic decays of the $\tau^{-}$involve no strong interactions at lowest order in charged weak coupling, and their properties are predicted very precisely by standard electroweak theory, allowing for precise checks of the standard model. Since the $\tau^{-}$lepton is massive enough to decay into hadrons, it also serves as an ideal environment for examination of the strong interaction.

This chapter provides an overview of the $\tau^{-}$lepton and its place within the standard model of particle physics. We review the existing measurements of $\tau^{-}$ branching fractions, and compare these experimental results with theoretical predictions.

\subsection{Particles and Interactions}

Elementary particle physics research is an effort to understand the fundamental nature of matter. Although matter, the stuff of physical phenomena, exhibits a diverse range of characteristics, particle physics research endeavors to describe all observed phenomena in terms of a few particles and their interactions.

Leptons

$$
\left(\begin{array}{c}
v_{e} \\
e
\end{array}\right)\left(\begin{array}{c}
v_{\mu} \\
\mu
\end{array}\right)\left(\begin{array}{l}
v_{\tau} \\
\tau
\end{array}\right)
$$

\section{Quarks}

$$
\left(\begin{array}{l}
u \\
d
\end{array}\right)\left(\begin{array}{l}
c \\
s
\end{array}\right)\left(\begin{array}{l}
t \\
b
\end{array}\right)
$$

Figure 2.1: Generations of leptons and quarks. 
The current standard model of particle physics enjoys remarkable success in its ability to describe physical interactions. Within this theory there are electroweak interactions $[5,6,7]$ mediated by the massless photon and the massive $W^{+}, W^{-}$, and $Z^{0}$ bosons, and strong interactions mediated by eight types of gluons. The matter particles are six flavors each of leptons and quarks, divided into three generations of weak isospin pairs, as shown in Figure 2.1. The leptons interact only through the electroweak force, while the quarks interact through both the electroweak and strong forces.

Each generation of leptons consists of one charged and one neutral particle. The lightest and only stable charged lepton is the electron. The quarks interact strongly and are not ooservable as free particles. They occur as bound states of $q \bar{q}$, known as mesuns, and $q q q$, known as karyons. For example, the proton is a bound state of uud while the neutron is a bound state of udd quarks.

\subsection{The Tau Lepton}

The $\tau^{-}$is the third generation charged lepton. It has a much shorter lifetime [8] than the lighter $\mu^{-}$lepton, and is observed indirectly through its decay products. The existence of the $\tau^{-}$was first established [9] through the process $e^{+} e^{-} \rightarrow \tau^{+} \tau^{-}$, where one of the $\tau$ decays to $e$ and neutrinos while the other $\tau$ decays to $\mu$ and neutrinos. The energy distributions of the observed $e$ and $\mu$ particles favored the hypothesis of a third generation lepton as the source for these decays.

Inherent in the standard model is the concept of lepton universality, which predicts that the three generations of charged leptons $\left(e^{-}, \mu^{-}, \tau^{-}\right)$have identical couplings to the intermediate bosons of the electroweak interaction $\left(\gamma, Z^{0}, W^{+}\right.$, $W^{-}$). The form of the electroweak lepton current is given exactly, and allows specific predictions of not only decay rates, but also the distribution of decay particles in phase space for the leptonic decays of the $\tau^{-}$. For hadronic decays, although the lepton half of the decay is predicted, we must use additional arguments to parameterize the weak hadronic current for any given set of final state particles.

\subsection{Decays of the Tau Lepton}

Within the standard model, the only flavor-changing interaction is that of the charged weak $W^{-}$intermediate boson. Interactions through the $\gamma$, the $Z^{0}$, or the gluons of the strong interaction do not alter the flavor of the lepton or quark current. Therefore, although bound states of quarks (mesons and baryons) may decay through the rearragement of quarks or the creation of new quark pairs, fundamental particles (quarks and leptons) decay through the $W^{-}$boson. 


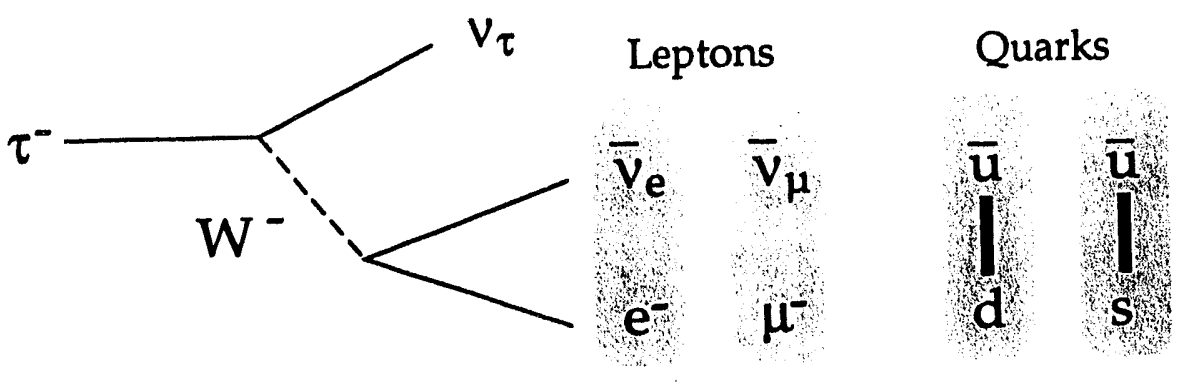

Figure 2.2: Particle doublets at the $W^{-}$vertex in $\tau^{-}$decays.

The coupling of the $\tau^{-}$current to the $W^{-}$boson of the weak interaction, shown in Figure 2.2, produces a weakly interacting tau neutrino, which is not directly observed but is inferred from missing energy in the $\tau^{-}$decay products. The virtual $W^{-}$created in this reaction then couples to an additional pair of leptons, $\bar{\nu}_{e} e^{-}$or $\bar{\nu}_{\mu} \mu^{-}$, or quarks, $\bar{u} d$ or $\bar{u} s$. All other quark pairings, such as $\bar{c} d$ or $\bar{c} s$, are too massive to be produced from $\tau^{-}$lepton decays. Therefore, to lowest order all decays of the $\tau^{-}$are included in these four processes,

$$
\begin{aligned}
& \tau^{-} \rightarrow \nu_{\tau} \bar{\nu}_{e} e^{-} \\
& \tau^{-} \rightarrow \nu_{\tau} \bar{\nu}_{\mu} \mu^{-} \\
& \tau^{-} \rightarrow \nu_{\tau} \bar{u} d / \bar{u} d \rightarrow \text { hadrons } \\
& \tau^{-} \rightarrow \nu_{\tau} \bar{u} s / \bar{u} s \rightarrow \text { hadrons }
\end{aligned}
$$

Note that the quarks which couple to the $W^{-}$may be from the same generation, as in the $u d$ case, or from different generations, as in the $\bar{u} s$ case. The relative strengths of these couplings are given by the elements of the Cabibbo-KobayashiMaskawa (CKM) matrix [8], $\left|V_{u d}\right|$ and $\left|V_{u s}\right|$. Couplings to quarks within generations are highly favored over couplings across generations. Consequently, the $\bar{u} s$ decays of the $\tau^{-}$have much smaller branching fractions than the analogous $\bar{u} d$ decays. This mixing between generations has not been observed for the lepton sector.

A list of decays of the $\tau^{-}$lepton is given in Table 2.1, along with experimental $[10,8]$ and theoretical values for their branching fractions. These decays are grouped according to the four possible pairs of particles produced at the $W^{-}$vertex. For the leptonic decays, there is only one decay mode for each of the possible lepton pairs. For the hadronic decays, the $\bar{u} d$ or $\bar{u} s$ quarks undergo a process of strong interactions in which additional $\bar{u} u, \bar{d} d$, or $\bar{s} s$ quark pairs may be created. The net result of this process is the production of some number of mesons, predominantly $\pi$ and $K$, both charged and neutral. Table 2.1 separates the $\bar{u} d$ and $\bar{u} s$ decays of the $\tau^{-}$into decay classes based on the numbers of $\pi$ and $K$ in the final state. 


\begin{tabular}{|c|c|c|c|c|c|}
\hline \multicolumn{4}{|c|}{ Tau Decay } & \multicolumn{2}{|c|}{ Branching Fraction(\%) } \\
\hline Vertex & $\pi / K$ Class & Channel & Final State & Experiment & Theory \\
\hline $\bar{\nu}_{e} e^{-}$ & & & $e^{-}$ & $17.89 \pm 0.14$ & $18.13 \pm 0.20$ \\
\hline $\bar{\nu}_{\mu} \mu^{-}$ & & & $\mu^{-}$ & $17.34 \pm 0.16$ & $17.64 \pm 0.19$ \\
\hline \multirow[t]{13}{*}{$\bar{u} d$} & $(\pi)^{-}$ & $\pi^{-}$ & $\pi^{-}$ & $11.6 \pm 0.4$ & $10.90 \pm 0.12$ \\
\hline & $(\pi \pi)^{-}$ & $\rho^{-}$ & $\pi^{-} \pi^{0}$ & $22.2 \pm 1.0$ & \\
\hline & $(\pi \pi \pi)^{-}$ & $a_{1}^{-}$ & $\pi^{-} \pi^{+} \pi^{-}$ & $(8.2 \pm 0.6)$ & \\
\hline & & & $\pi^{-} \pi^{0} \pi^{0}$ & $(9.0 \pm 0.8)$ & \\
\hline & $(\pi \pi \pi \pi)^{-}$ & & $\pi^{-} \pi^{+} \pi^{-} \pi^{0}$ & $(4.8 \pm 0.6)$ & \\
\hline & & & $\pi^{-} \pi^{0} \pi^{0} \pi^{0}$ & $(1.8 \pm 0.5)$ & \\
\hline & $(\pi \pi \pi \pi \pi)^{-}$ & & $\pi^{-} \pi^{+} \pi^{-} \pi^{+} \pi^{-}$ & $(0.08 \pm 0.02)$ & \\
\hline & & & $\pi^{-} \pi^{+} \pi^{-} \pi^{0} \pi^{0}$ & $(0.95 \pm 0.12)$ & \\
\hline & & & $\pi^{-} \pi^{0} \pi^{0} \pi^{0} \pi^{0}$ & $(0.15 \pm 0.07)$ & \\
\hline & $(K K)^{-}$ & & $K^{-} K^{0}$ & $<0.26$ & \\
\hline & $(K K \pi)^{-}$ & & $K^{-} K^{+} \pi^{-}$ & $0.22 \stackrel{+0.17}{-0.11}$ & \\
\hline & & & $K^{-} K^{0} \pi^{0}$ & & \\
\hline & & & $\pi^{-} K^{0} \bar{K}^{0}$ & & \\
\hline \multirow[t]{7}{*}{$\bar{u} s$} & $(K)^{-}$ & $K^{-}$ & $K^{-}$ & $0.67 \pm 0.23$ & $0.73 \pm 0.01$ \\
\hline & $(K \pi)^{-}$ & $K^{*-}$ & $K^{-} \pi^{0}$ & & \\
\hline & & & $\pi^{-} \bar{K}^{0}$ & $0.95 \pm 0.12$ & \\
\hline & $(K \pi \pi)^{-}$ & $K_{1}^{-}$ & $K^{-} \pi^{+} \pi^{-}$ & $0.22 \pm_{-0.13}^{+0.16}$ & \\
\hline & & & $K^{-} \pi^{0} \pi^{0}$ & & \\
\hline & & & $\pi^{-} \bar{K}^{0} \pi^{0}$ & & \\
\hline & $(K K K)^{-}$ & & $\begin{array}{l}K^{-} K^{+} K^{-} \\
K^{-} K^{0} \bar{K}^{0}\end{array}$ & $<0.17$ & \\
\hline
\end{tabular}

Table 2.1: $\tau^{-}$decays and branching fractions. Decays are classified according to the particles at the $W^{-}$vertex and the numbers of $\pi$ and $K$ in the final state. Experimental values are current world averages, excluding the measurements presented in this thesis. Theoretical values are based on $\tau^{-}$mass and lifetime measurements. The branching fraction measurements in parentheses are estimated from experiments that do not distinguish charged $\pi$ and $K$.

\subsection{Leptonic Decays}

Rates for the decays of the $\tau^{-}$to leptons are predicted to lowest order by standard electroweak theory. Since the leptons exhibit no strong interaction, there are only very small corrections to this basic formula. The matrix element for the decay of 
a charged lepton $L^{-}$to $\nu_{L}$ and another lighter lepton pair, $l^{-}$and $\bar{\nu}_{l}$, is

$$
\mathcal{M}=\frac{G_{F}}{\sqrt{2}}\left[\bar{u}_{\nu_{L}} \gamma^{\mu}\left(1-\gamma^{5}\right) u_{L}\right]\left[\bar{u}_{l} \gamma_{\mu}\left(1-\gamma^{5}\right) v_{\nu_{l}}\right]
$$

Since the $W^{-}$is so far off mass shell for decays at the mass of the known leptons, the $W^{-}$propagator is essentially a constant and is combined with the weak coupling constant to give $G_{F}$, the Fermi coupling constant determined from $\mu^{-}$decay. In the limit of negligible mass for the decay products, the integration of this matrix element over phase space is [11],

$$
\Gamma\left(L \rightarrow \nu_{L} l \bar{\nu}_{l}\right)=\frac{G_{F}^{2} m_{L}^{5}}{192 \pi^{3}}
$$

where $m_{L}$ is the mass of the decaying lepton. The inclusion of radiative corrections and the non-local structure of the $W^{-}$propagator introduces an additional factor [12],

$$
r=\left[1+\frac{\alpha}{2 \pi}\left(\frac{25}{4}-\pi^{2}\right)\right]\left[1+\frac{3}{5} \frac{m_{L}^{2}}{m_{W}^{2}}\right]=0.9957
$$

for $m_{L}=m_{\tau}$. These formulas provide a useful reference for describing theoretical predictions of the relative rates for decays of the $\tau^{-}$lepton. For the leptonic decays of the $\tau^{-}$, there are small corrections for the mass of the $e^{-}$or $\mu^{-}$which give values of

$$
\begin{aligned}
\Gamma\left(\tau^{-} \rightarrow \nu_{\tau} \bar{\nu}_{\mathrm{e}} e^{-}\right) & =1.000 \Gamma_{0} \\
\Gamma\left(\tau^{-} \rightarrow \nu_{\tau} \bar{\nu}_{\mu} \mu^{-}\right) & =0.973 \Gamma_{0}
\end{aligned}
$$

where the reference decay rate is given by,

$$
\Gamma_{0}=\left(\frac{G_{F}^{2} m_{\tau}^{5}}{192 \pi^{3}}\right) r=4.035 \pm 0.006 \times 10^{-4} \mathrm{eV}
$$

with an error dominated by the world average value for measurements of the $\tau^{-}$ mass of [13],

$$
m_{\tau}=1777.1 \pm 0.5 \mathrm{MeV}
$$

In order to convert the predicted leptonic partial decay rates into branching fractions, we use the total decay rate

$$
\Gamma_{\tau}=\frac{1}{\tau_{\tau}}=22.26 \pm 0.24 \times 10^{-4} \mathrm{eV}
$$

determined from measurements of the $\tau^{-}$lifetime [14],

$$
\tau_{\tau}=295.7 \pm 3.2 \mathrm{fs}
$$


Combining the total and partial decay rate predictions gives,

$$
\begin{aligned}
B\left(\tau^{-} \rightarrow \nu_{\tau} \bar{\nu}_{e} e^{-}\right) & =18.13 \pm 0.20 \% \\
B\left(\tau^{-} \rightarrow \nu_{\tau} \bar{\nu}_{\mu} \mu^{-}\right) & =17.64 \pm 0.19 \%
\end{aligned}
$$

The branching fractions predicted from theory for the leptonic decays of the $\tau^{-}$are at the $1 \%$ error level, thanks to recent precise measurements of the $\tau^{-}$mass and lifetime. There already exists a large number of experimental measurements of the leptonic decays of the $\tau^{-}$. The relative errors of the world average values are also at the $1 \%$ level. The examination of these decay modes has reached the level of precision necessary to make careful checks of standard electroweak theory. Unfortunately, the size of our data sample limits us to measurements at the $5 \%$ level of precision and prevents us from making a significant contribution for leptonic branching fractions.

\subsection{Hadronic Decays}

Hadronic decays, in which the $W^{-}$couples to $\bar{u} d$ or $\bar{u} s$, are much more complicated than the leptonic decays. The quarks are not observed as free particles but undergo strong interactions which produce some number of hadrons, bound states of quarks. Conservation of quark currents requires that any baryonic decays of the $\tau^{-}$would include both a baryon and an anti-baryon. The mass of the lightest charge-conserving baryon pair, a neutron and anti-proton, exceeds that of the $\tau^{-}$ lepton by about $100 \mathrm{MeV}$. Consequently, hadronic decays of the $\tau^{-}$produce only mesons. The following sections take a closer look at the process of strong interactions that begins with the creation of a $\bar{u} d$ or $\bar{u} s$ quark pair at the $W^{-}$vertex, and results in a final state of long-lived mesons.

\subsubsection{Resonances}

Hadronic decays of the $\tau^{-}$lepton occur predominantly through direct coupling of the $W^{-}$to resonant states of $\bar{u} d$ and $\bar{u} s$ quarks. The coupling strength of the $\bar{u} s$ relative to that of the $\bar{u} d$ is estimated from the square of the relevant CKM matrix elements, $\left|V_{u s}\right|^{2}$ and $\left|V_{u d}\right|^{2}$, which predict that $\bar{u} s$ decays will occur at about $5 \%$ the rate of $\bar{u} d$ decays.

The possible $\bar{u} d$ and $\bar{u} s$ resonant states are shown in Figure 2.3 and Figure 2.4. For the $\bar{u} d$ coupling, $\tau^{-}$decays are dominated by the pseudoscalar $\pi^{-}\left(0^{-+}\right)$, vector $\rho^{-}\left(1^{--}\right)$, and axial-vector $a_{1}^{-}\left(1^{++}\right)$resonances, while the scalar $a_{0}^{-}\left(0^{++}\right)$and axialvector $b_{1}^{-}\left(1^{+-}\right)$decays are not observed. This pattern is predicted by the standard model, and the $J^{P C}$ states of the $\bar{u} d$ quark system that do not couple to the $W^{-}$ are referred to as second class currents $[6,15]$. 


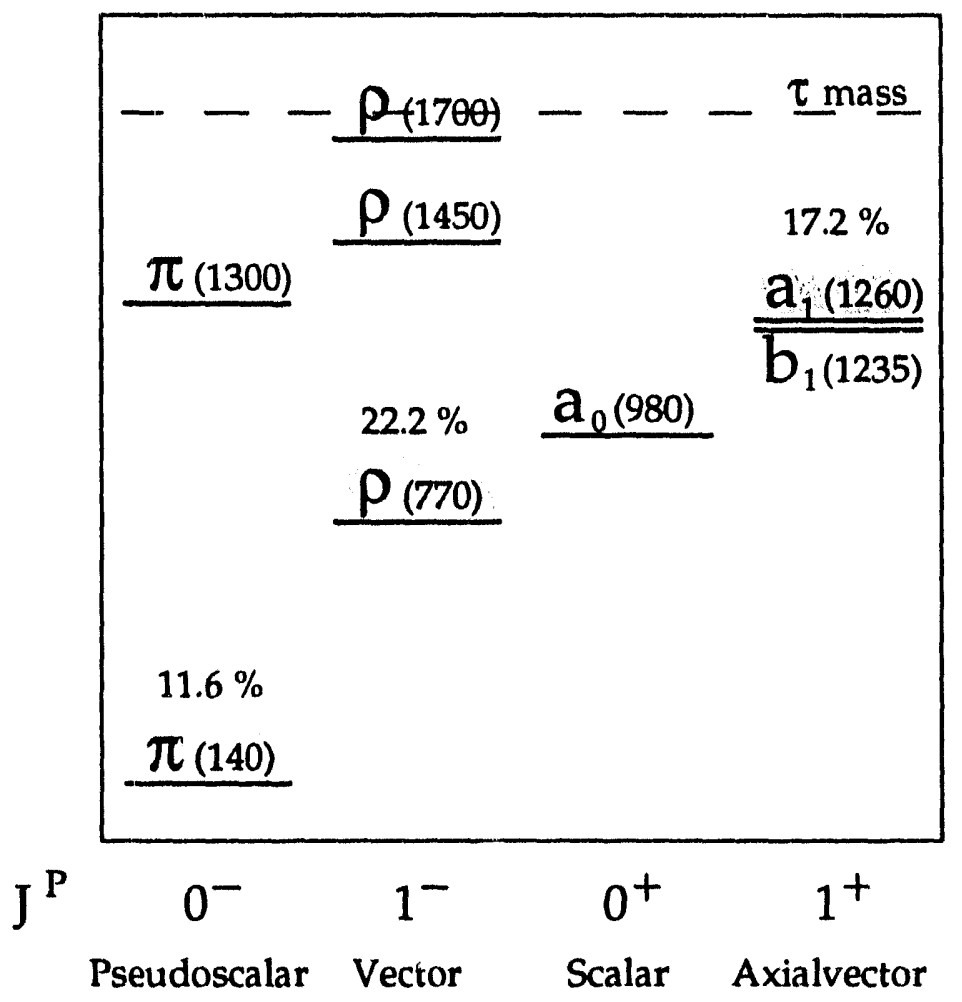

Figure 2.3: Spectroscopy of $\bar{u} d$ quark states. The states that are observed to dominate the $\bar{u} d$ decay channels of the $\tau^{-}$are shaded, with branching fractions listed above.

The $\bar{u} s$ pseudoscalar and vector decays parallel the $\bar{u} d$ analogs, and are dominated by the the $\mathrm{K}^{-}\left(0^{-+}\right)$and $\mathrm{K}^{*-}\left(1^{--}\right)$channels. The ratio of the $\bar{u} s / \bar{u} d$ decay rates for pseudoscalar and vector decays is at the predicted $5 \%$ level. However, the pattern of coupling for the $\bar{u} s$ axial-vector states is complicated by the fact that the $s$ quark mass is considerably larger than the lighter $u$ quark and $d$ quark masses, and breaks the $\mathrm{SU}(3)$ flavor symmetry. This allows coupling of both $\bar{u} s$ axial-vector states $\left(1^{++}, 1^{+-}\right)$to the $W^{-}$. In addition, these $\bar{u} s$ analogs of the $a_{1}^{-}$ and $b_{1}^{-}$mix to produce the observed $K_{1}^{-}(1270)$ and $K_{1}^{-}(1400)$ states. Thus, while the $a_{1}^{-}$alone is observed to dominate the $\bar{u} d$ axial-vector decay of the $\tau^{-}$, both the $K_{1}^{-}(1270)$ and $K_{1}^{-}(1400)$ are possible candidates for the $\bar{u} s$ axial-vector decay.

This thesis includes the first measurements of the branching fractions for the $\bar{u} s$ axial-vector decays, $\tau^{-} \rightarrow \nu_{\tau} K_{1}^{-}(1270)$ and $\tau^{-} \rightarrow \nu_{\tau} K_{1}^{-}(1400)$. The net decay rate for $\tau^{-} \rightarrow \nu_{\tau} K_{1}^{-}$is found to be about $5 \%$ of that for $\tau^{-} \rightarrow \nu_{\tau} a_{1}^{-}$, as expected from the relative magnitude of the CKM matrix elements, $\left|V_{u s}\right|^{2}$ and $\left|V_{u d}\right|^{2}$. 


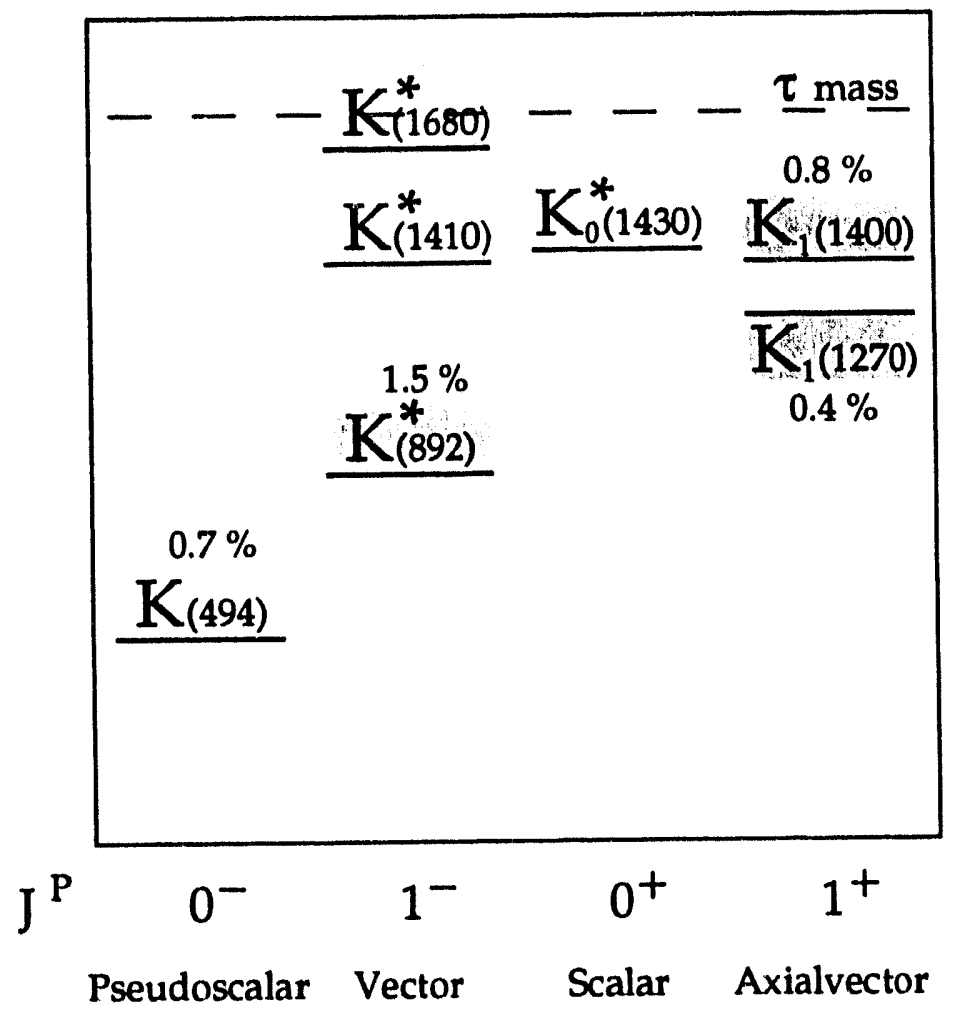

Figure 2.4: Spectroscopy of $\bar{u} s$ quark states. The states that are observed to dominate the $\bar{u} s$ decay channels of the $\tau^{-}$are shaded, with branching fractions listed. The first observation and measurement of the $K_{1}^{-}$decays are described in this thesis.

\subsubsection{Final States}

We now consider the possible final states of the hadronic decays of the $\tau^{-}$, classified according to the number of $\pi$ and $K$ mesons present.

$\tau^{-} \rightarrow \nu_{\tau} \pi^{-}$and $\tau^{-} \rightarrow \nu_{\tau} K^{-}$

These $\bar{u} d$ and $\bar{u} s$ pseudoscalar decays are the simplest of the hadronic decays. Although the strong interactions preclude an exact calculation from first principles, the weak hadronic current may be parameterized very easily since the momentum of the meson is the only four-vector available for these spinless particles. The currents are of the form,

$$
\begin{aligned}
\left\langle\pi^{-}\left|\bar{d} \gamma^{\mu} \gamma^{5} u\right| \emptyset\right\rangle & \equiv i \sqrt{2} f_{\pi}\left|V_{u d}\right| \frac{p^{\mu}}{m_{\pi}} \\
\left\langle K^{-}\left|\bar{s} \gamma^{\mu} \gamma^{5} u\right| \emptyset\right\rangle & \equiv i \sqrt{2} f_{K}\left|V_{u s}\right| \frac{p^{\mu}}{m_{K}}
\end{aligned}
$$


where the coupling constants $f_{\pi}$ and $f_{K}$ are measured very precisely from the decays $\pi^{-} \rightarrow \bar{\nu}_{\mu} \mu^{-}$and $K^{-} \rightarrow \bar{\nu}_{\mu} \mu^{-}$[8]. Additional electroweak radiative corrections predict the decay rates of [12],

$$
\begin{aligned}
\Gamma\left(\tau^{-} \rightarrow \nu_{\tau} \pi^{-}\right) & =0.601 \Gamma_{0} \\
\Gamma\left(\tau^{-} \rightarrow \nu_{\tau} K^{-}\right) & =0.040 \Gamma_{0}
\end{aligned}
$$

These are the only hadronic decays of the $\tau^{-}$that are predicted with such accuracy.

The current experimental values for these decays are consistent with the theoretical predictions, although the relative errors on these measurements are much larger than those of the leptonic modes since few experiments are capable of distinguishing $\pi^{ \pm}$and $K^{ \pm}$.

$\tau^{-} \rightarrow \nu_{\tau}(\pi \pi)^{-}$and $\tau^{-} \rightarrow \nu_{\tau}(K \pi)^{-}$

These decays proceed through the $\rho^{-}$and $K^{*-}$ vector channels [16]. The $(\pi \pi)^{-}$ decay has the largest branching fraction of any single decay of the $\tau^{-}$, making up about $25 \%$ of the total decay rate. The $(\pi \pi)^{-}$decay has only one mode, $\pi^{-} \pi^{0}$. The most difficult aspect of measuring the $\pi^{-} \pi^{0}$ branching fraction is the separation of events with exactly one $\pi^{0}$ from those with none or two. Also, as the measurements become more precise, experiments must be able to separate out the small $K^{-} \pi^{0}$ background to this decay mode.

The $(K \pi)^{-}$decay may proceed to either of two final states, $K^{-} \pi^{0}$ or $\pi^{-} \bar{K}^{0}$. Although the $K^{ \pm}$has a sufficiently long lifetime to allow observation in tracking devices, few experiments are able to identify the few $K^{ \pm}$among the many $\pi^{ \pm}$. The alternative is to reconstruct the $\bar{K}^{0}$ from its decay to $\pi^{+} \pi^{-}$. The states $K^{0}$ and $\bar{K}^{0}$ mix weakly to give observed states known as $K_{S}^{0}$ and $K_{L}^{0}$, according to their short and long lifetimes. The $K_{L}^{0}$, which makes up half of the neutral $K^{0}$ and $\bar{K}^{0}$, will usually pass through the detector without observation. On the other hand, the $K_{S}^{0}$ decay to $\pi^{-} \pi^{+}$or $\pi^{0} \pi^{0}$. The existing experimental measurements for the $K^{*-}$ decay are based on the reconstruction of the neutral $K_{S}^{0}$. In chapter 5 we present a measurement based on the $K^{-} \pi^{0}$ mode.

$$
\tau^{-} \rightarrow \nu_{\tau}(\pi \pi \pi)^{-} \text {and } \tau^{-} \rightarrow \nu_{\tau}(K \pi \pi)^{-}
$$

The $(\pi \pi \pi)^{-}$decays follow the pattern of resonance dominance and are observed to proceed through the $a_{1}^{-}$axial-vector channel [17]. There are two modes, $\pi^{-} \pi^{+} \pi^{-}$ and $\pi^{-} \pi^{0} \pi^{0}$, which are expected to occur in a 1:1 ratio, given the $a_{1}^{-}$dominance. Unfortunately there are no measurements of these decay modes from experiments with $\pi^{ \pm} / K^{ \pm}$particle identification. The sum of experimental values for the decays $h^{-} h^{+} h^{-}(8.2 \pm 0.6 \%)$ and $h^{-} \pi^{0} \pi^{0}(9.0 \pm 0.8 \%)$, where $h$ represents either $\pi$ or $K$, may be used to estimate the $a_{1}^{-}$branching fraction and to check the 1:1 ratio of one-prong and three-prong contributions. 
This thesis provides first evidence that the $(K \pi \pi)^{-}$decays proceed through the $K_{1}^{-}(1270)$ and $K_{1}^{-}(1400)$ channels, with a net branching fraction of $1.17 \pm 0.41 \%$. The measurement is based on the all-charged $K^{-} \pi^{+} \pi^{-}$mode.

$\tau^{-} \rightarrow \nu_{\tau}(\pi \pi \pi \pi)^{-}$and $\tau^{-} \rightarrow \nu_{\tau}(\pi \pi \pi \pi \pi)^{-}$

As the number of $\pi$ in the final state increases and the available phase space decreases, the corresponding branching fractions are observed to drop off. The four $-\pi$ modes are measured to make up about $6 \%$ of the total $\tau^{-}$decay rate, while the five- $\pi$ modes combine to give a net branching fraction of about $1 \%$.

$\tau^{-} \rightarrow \nu_{\tau}(K K)^{-}$and $\tau^{-} \rightarrow \nu_{\tau}(K K \pi)^{-}$

These decays each have an even number of $K$, indicating that the $W^{\prime}$ vertex coupling is to $\bar{u} d$ with the subsequent creation of an $\bar{s} s$ quark pair from the vacuum. The $(K K)^{-}$decay has a single mode, $K^{-} K^{0}$, which requires both $\pi^{ \pm} / K^{ \pm}$particle identification and reconstruction of $K_{S}^{0}$. Note that while the $\pi^{-} \pi^{0}$ decay has the largest branching fraction of any individual mode, the $K^{-} K^{0}$ decay has an upper limit of $<0.26 \%$ [18]. This suppression of $\bar{K} K$ creation is due to both resonance and phase space effects. The $\rho^{-}$, which dominates the $\pi^{-} \pi^{0}$ decay, is too light to decay to $K^{-} K^{0}$. Also, the relatively high mass of the $K$ compared to the $\pi$, results in a phase space suppression for states with $\bar{K} K$ pairs.

Similarly, the all-charged $K^{-} K^{+} \pi^{-}$mode of the $(K K \pi)^{-}$decay, measured to be $0.22+0.17$ [19], may be compared with the $\pi^{-} \pi^{+} \pi^{-}$branching fraction of $8.2 \pm 0.6$ to obtain an estimate of the suppression of modes with $\bar{K} K$ pairs.

$\tau^{-} \rightarrow \nu_{\tau}(K K K)^{-}$

The three $K$ here come from $W^{-}$vertex coupling to $\bar{u} s$ and the creation of an $\bar{s} s$ quark pair from the vacuum. Note that the mass of the $\tau^{-}$limits the number of $K$ in any final state to three.

\subsubsection{Quark Model of Mesons}

In the preceeding discussion, we have considered only those final states that consist of various combinations of charged and neutral $\pi$ and $K$. Here we make a brief excursion to discuss the quark model of mesons, and consider a more complete list of mesons that may possibly appear in the hadronic decay channels of the $\tau^{-}$.

Although there is one stable baryon, the proton, there are no stable mesons. The more massive mesons typically decay strongly to lighter mesons. These lighter mesons eventually decay through electroweak interactions to leptons, neutrinos, 


\begin{tabular}{|c|c|c|c|c|c|c|}
\hline & & eson & ctros & & & \\
\hline & & & & $J^{P C}$ & & \\
\hline & $q \bar{q}$ & $0^{-+}$ & $1^{--}$ & $0^{++}$ & $1^{++}$ & $1^{+-}$ \\
\hline & $\bar{u} d$ & $\pi^{-}$ & $\rho^{-}$ & $a_{0}^{-}$ & $a_{1}^{-}$ & $b_{1}^{-}$ \\
\hline & $\bar{d} u$ & $\pi^{+}$ & $\rho^{+}$ & $a_{0}^{+}$ & $a_{1}^{+}$ & $b_{1}^{+}$ \\
\hline Flavored Charged & $\bar{u} s$ & $K^{-}$ & $K^{*-}$ & $K_{0}^{*-}$ & $K_{a 1}^{*-}$ & $K_{b 1}^{*-}$ \\
\hline & $\overline{\boldsymbol{s}} u$ & $k^{+}$ & $K^{*+}$ & $K_{0}^{*+}$ & $K_{a 1}^{*+}$ & $K_{b 1}^{*-}$ \\
\hline Flaugred Noutral & $\overline{d s}$ & $\bar{K}^{0}$ & $\bar{K} * 0$ & $\bar{K}_{0}^{* 0}$ & $\bar{K}_{a 1}^{* 0}$ & $\bar{K}_{b 1}^{* 0}$ \\
\hline Flavored Neutral & $\bar{s} d$ & $K^{0}$ & $K^{* 0}$ & $K_{0}^{* 0}$ & $K_{a 1}^{* 0}$ & $K_{b 1}^{* 0}$ \\
\hline & $\bar{u} u$ & $\pi^{0}$ & $\rho^{0}$ & $a_{0}^{0}$ & $a_{1}^{0}$ & $b_{1}^{0}$ \\
\hline Flavorless Neutral & $\bar{d} d$ & $\eta$ & $\omega$ & $f_{0}$ & $f_{1}$ & $h_{1}$ \\
\hline & $\bar{s} s$ & $\eta^{\prime}$ & $\phi$ & $f_{0}^{\prime}$ & $f_{1}^{\prime}$ & $h_{1}^{\prime}$ \\
\hline
\end{tabular}

Table 2.2: $q \tilde{q}$ flavor and $J^{P C}$ states. The three flavorless neutral states mix strongly, while the two flavored neutral states mix weakly.

and photons. Some of these mesons have lifetimes long enough to allow direct observation while others must be reconstructed from their decay products. More massive mesons tend to have shorter lifetimes, broader widths, and more complex decays. This makes reconstruction progressively more difficult with increasing mass.

From the properties of the $\tau^{-}$lepton and weak interactions, there are constraints that we can place on the nature of the mesons that are produced in $\tau^{-}$ decays. Although the number of neutral mesons is not fixed, the number of charged mesons must be odd to conserve the charge of the $\tau^{-}$. Unlike the leptonic decays, which are necessarily one-prong decays, the hadronic decays may be one-prong, three-prong, five-prong, or of even higher charged multiplicity.

The mass of the $\tau^{-}$limits the possible flavors of the quarks within the mesons to $d, u$, or $s$. This gives nine possible flavor states for the $q \bar{q}$ mesons, organized by flavor and charge in Table 2.2, which also lists the ground state mesons for several $J^{P C}$ combinations. Note that the flavorless neutral states $\bar{u} u, \bar{d} d$, and $\bar{s} s$ mix strongly to produce the physically observed states. Also, the neutral flavored $\bar{d} s$ and $\bar{s} d$ states may mix weakly. This is relevant for the $K^{0}$ and $\bar{K}^{0}$ which decay weakly as $K_{S}^{0}$ and $K_{L}^{0}$.

\section{Flavored Charged Mesons}

Four of the flavor states have net flavor and charge. These are the $\bar{u} d, \bar{u} s$, and their anti-particles. These states are of particular interest since their quark contents 
match those possible at the $W^{-}$vertex. Thus they potentially couple directly to the $W^{-}$boson and may represent the starting point for the hadronic processes observed in decays of the $\tau^{-}$. The long-lived pseudoscalar ground states, $\pi^{ \pm}$and $K^{ \pm}$, decay weakly since they are the lightest states with net flavor. All other states decay strongly to lighter mesons.

\section{Flavored Neutral Mesons}

The $\bar{d} s$ and $\bar{s} d$ states have net flavor but are electrically neutral. Although they do not couple directly to the $W^{-}$, they may appear as decay products of higher level resonances. The pseudoscalar and vector ground states, $K^{0}$ and $K^{* 0}$, have been observed in $\tau^{-}$decays. Since these states are the neutral members of isospin doublets with the $\bar{u} s$ and $\bar{s} u$ states, we can make inferences about the relative numbers of neutral and charged $K$ in a sample of $\tau^{-}$decays for a given channel. For example, $K^{*-}$ decays to $\bar{K}^{0} \pi^{-}$or $K^{-} \pi^{0}$ in a $2: 1$ ratio. Therefore we can estimate the number of $\tau^{-}$decays to $\nu_{\tau} K^{*-}$ from either final state.

\section{Flavorless Neutral Mesons}

These states mix strongly to give physical particles that are linear combinations of $\bar{u} u, \bar{d} d$, and $\bar{s} s$. Due to the near mass degeneracy of the $d$ and $u$ quark, one of these linear combinations, $\sqrt{\frac{1}{2}}(\bar{u} u-\bar{d} d)$, will be the third member of an isospin triplet with the flavored charged $\bar{u} d$ and $\bar{d} u$ states, and will have similar mass. Since these states have no net flavor, they may decay through the neutral currents of the electroweak interaction.

\subsubsection{Photons in Tau Decays}

The photon is a stable particle that may be observed through the electromagnetic shower it produces in dense materials. Here we consider the possible sources for photons in $\tau^{-}$decays.

Most of the energetic photons observed in $\tau^{-}$decays are from the decays of $\pi^{0}$. More than half of the $\tau^{-}$decay rate is made up of decay modes that include at least one $\pi^{0}$. There are existing measurements for branching fractions of $\tau^{-}$decays with one, two, and three reconstructed $\pi^{0}$, and the study of decays with multiple $\pi^{0}$ continues to be an active area of research.

Another possible source for photons is the $\eta$, which like the $\pi^{0}$ decays largely to photons. Searches for the $\eta$ in $\tau^{-}$decays have placed an upper limit of $0.5 \%$ on the inclusive branching fraction. The vector analog $\omega$ meson is observed in the decay $\tau^{-} \rightarrow \nu_{\tau} \pi^{-} \omega$ with a branching fraction of $1.6 \pm 0.5 \%$. The $\omega$ has a branching 
fraction to $\pi^{0} \gamma$ of $8.5 \%$, which indicates that $0.1 \%$ of $\tau^{-}$decays will have a photon from $\omega$ decay.

Since the contribution of photons from $\eta$ and $\omega$ decays is so small, the approach of global measurements of $\tau^{-}$branching fractions has been to classify the neutral portion of the decay as an estimate of the number of $\pi^{0} s$, based on fully reconstructed $\pi^{0} \mathrm{~s}$ and the number of additional energetic photons. We will adhere to this convention in our global analysis.

\subsection{Discussion}

This chapter has given a brief overview of the physics of the $\tau^{-}$lepton and a survey of its decay modes. The leptonic modes are relatively simple decays that produce a single charged lepton, $e^{-}$or $\mu^{-}$, in the final state. The current theoretical and experimental values for these branching fractions are both known with a precision of about $1 \%$ and are consistent.

The hadronic decays may be divided according to the $W^{-}$vertex coupling into $\bar{u} d$ and $\bar{u} s$ decays. Reconstruction of the various decay channels that lead from the initial $\bar{u} d$ or $\bar{u} s$ creation to the final state of charged and neutral $\pi$ and $K$ provides insight into the strong interaction. The measurements for the branching fractions of many of the hadronic decays are at the level of precision where the $K$ contributions are no longer negligible. Thus, a more thorough understanding of hadronic $\tau^{-}$decays requires detectors that are able to distinguish $\pi^{ \pm}$and $K^{ \pm}$, and to reconstruct $\pi^{0}$ and $K^{0}$. 


\section{Chapter 3}

\section{The TPC/Two-Gamma Experiment}

The measurements described in this thesis are based on data collected with the TPC/Two-Gamma detector situated at the PEP $e^{+} e^{-}$collider of the Stanford Linear Accelerator Center (SLAC). This chapter begins with an overview of the accelerator system and of the characteristics of the various types of events observed in $e^{+} e^{-}$interactions at the center of mass energy of PEP. We then give a description of the detector components and their capabilities, and conclude with a summary of the process of event reconstruction.

\subsection{SLAC and the PEP Collider}

During its 1982-1983 and 1984-1986 experimental runs, the TPC/Two-Gamma detector accumulated $140 \mathrm{pb}^{-1}$ of data at an $e^{+} e^{-}$center of mass energy of $29 \mathrm{GeV}$. A diagram of the SLAC linear accelerator and the PEP collider, used to produce and store the high energy bunches of electrons and positrons, is shown in Figure 3.1. Electrons were accelerated to an energy of $14.5 \mathrm{GeV}$ along the $3 \mathrm{~km}$ path of the linear accelerator by intense electric fields produced by a series of microwave klystrons. These electrons were then steered into orbits within the PEP storage ring. The acceleration of electrons was synchronized to produce three bunches, evenly spaced along the storage ring of the PEP collider. Positrons, generated by a tungsten target located along the linear accelerator, were also accelerated to $14.5 \mathrm{GeV}$ and then steered irto three bunches, orbiting in the direction opposite to that of the electrons. The configuration of $e^{+}$and $e^{-}$bunches allowed for six interaction points where the paths of the bunches would intersect. The $2.2 \mathrm{~km}$ circumference of the PEP collider gave an $e^{+} e^{-}$crossover period of roughly $2.4 \mu \mathrm{s}$ at the interactions points. 


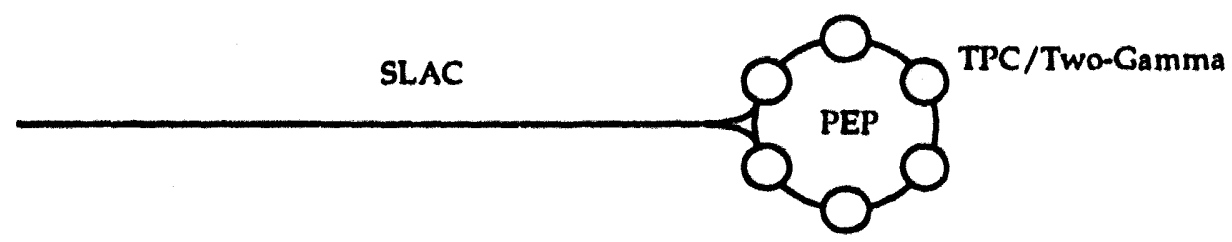

Figure 3.1: Diagram of the SLAC linear accelerator and PEP collider. Electrons were accelerated along the linac and stored in the PEP ring in three evenly spaced bunches. Similarly, positrons were accelerated and stored in three bunches with the direction of orbit opposite to that of the electrons. The circles show the locations of the interaction regions.

\subsection{Physics at PEP}

Electron-positron interactions produce remarkably clean events. The simplest process is scattering of an $e^{+} e^{-}$pair through exchange of a virtual photon,

$$
e^{+} e^{-} \rightarrow e^{+} e^{-}
$$

In annihilation events, the initial state $e^{+} e^{-}$pair interact through a virtual photon to create a final state pair of leptons or quarks according to,

$$
\begin{aligned}
& e^{+} e^{-} \rightarrow l^{+} l^{-} \\
& e^{+} e^{-} \rightarrow q \bar{q}
\end{aligned}
$$

The possible lepton pair flavors are $e^{+} e^{-}, \mu^{+} \mu^{-}$, and $\tau^{+} \tau^{-}$, while the possible quark pair flavors are $d \bar{d}, u \bar{u}, s \bar{s}, c \bar{c}$, and $b \bar{b}$. For a symmetric collider such as PEP, the resulting $l^{+} l^{-}$or $q \bar{q}$ pairs are produced at beam energy and separate collinearly. In addition to scattering and annihilation processes, there are also two-photon events, in which the $e^{+}$and $e^{-}$each couple to a virtual photon. These two virtual photons then couple to an additional lepton or quark pair according to,

$$
\begin{array}{lll}
e^{+} e^{-} \rightarrow e^{+} e^{-} \gamma^{\star} \gamma^{\star} & / & \gamma^{\star} \gamma^{\star} \rightarrow l^{+} l^{-} \\
e^{+} e^{-} \rightarrow e^{+} e^{-} \gamma^{\star} \gamma^{\star} & / & \gamma^{\star} \gamma^{\star} \rightarrow q \bar{q}
\end{array}
$$

Here the final state includes the original $e^{+} e^{-}$pair in addition to a new $l^{+} l^{-}$or $q \bar{q}$ pair.

Below is a brief survey of physics that takes place in the $e^{+} e^{-}$interactions at PEP energy. We present some of the characteristics used in the identification of the various event types. Note that all of these processes may include photons which are radiated by initial or final state particles. 
$e^{+} e^{-} \rightarrow e^{+} e^{-}(\gamma)$

This reaction, known as Bhabha scattering, is the most common type of event, with a differential cross section that diverges to infinity for small angles of deflection. There are two lowest order processes that contribute to this reaction. The cross section is dominated by the scattering process, in which the initial state electron and positron simply exchange a virtual photon. There is also an annihilation process in which the initial state $e^{+} e^{-}$combine to create a virtual photon, which then couples to the final state $e^{+} e^{-}$. In the radiative version of this reaction, an additional photon is emitted by one of the intial or final state $e^{+}$or $e^{-}$.

These events are characterized by a single pair of near-collinear electrons at or near beam energy. Consequently, identification is based on total energy, track topology, and particle identification. The radiative Bhabha events pose a particular problem since the tracks may not be collinear and the photon may convert to produce an additional $e^{+} e^{-}$pair that fakes a higher multiplicity event.

$e^{+} e^{-} \rightarrow \mu^{+} \mu^{-}(\gamma)$

Although the kinematics of this reaction are very similar to those for Bhabha scattering, the cross section is much smaller since only the annihilation process contributes. The muon is stable to all interactions except the weak and rarely decays within the volume of the detector at PEP energy.

These events are characterized by a single pair of near-collinear charged tracks at or near beam energy, that pass through the layers of the muon detection system. As with Bhabha events, identification is based on total energy, track topology, and particle identification.

$e^{+} e^{-} \rightarrow \tau^{+} \tau^{-}(\gamma)$

This reaction is identical to the $\mu^{+} \mu^{-}$reaction, except for the higher mass of the $\tau^{+} \tau^{-}$leptons. However, the $\tau$ typically survives less than a millimeter after its creation due to its much shorter lifetime. Since the decay of each of the $\tau$ leptons produces at least one neutrino, these events are characterized by missing energy and the charged tracks from the decays tend to be acollinear. However, at PEP energy the decay products of the $\tau$ are boosted enough to give excellent separation into distinct decay hemispheres. Since each of the $\tau$ decays produces an odd number of charged particles, $\tau^{+} \tau^{-}$events are categorized according to their charged track multiplicity. For example, the most common topologies for $\tau^{+} \tau^{-}$ events are referred to as $\tau_{1+1}$ and $\tau_{1+3}$ events. 
$e^{+} e^{-} \rightarrow q \bar{q}(\gamma)$

This reaction is identical to those for the $\mu^{+} \mu^{-}$and $\tau^{+} \tau^{-}$leptons. However, here the quarks interact strongly to produce some number of hadrons. These events tend to have much higher charged multiplicity than the QED events, with an average of ten charged particles in the final state at PEP energy.

$e^{+} e^{-} \rightarrow e^{+} e^{-} \gamma^{\star} \gamma^{\star} / \gamma^{\star} \gamma^{\star} \rightarrow l^{+} l^{-}$or $q \bar{q}$

These events are mediated by two virtual photons, which produce an additional $e^{+} e^{-}, \mu^{+} \mu^{-}, \tau^{+} \tau^{-}$, or $q \bar{q}$. The virtual photons tend to be near collinear with the beamline and the intial $e^{+}$and $e^{-}$deflect only slightly from their original path. Since much of the energy is carried away by the original $e^{+} e^{-}$, these events are characterized by large missing energy.

\subsection{The TPC/Two-Gamma Detector}

The TPC/Two-Gamma detector, as with all general purpose detectors, is actually a composite of many different detector systems [20]. The time projection chamber (TPC) tracks charged particles to give values of momentum and ionization. The electromagnetic calorimeters (CAL) detect and give energy estimates of photons. The muon chambers (MUC) detect muons which pass through materials that stop all other charged particles. Drawings of the detector are shown in Figure 3.2 and Figure 3.3. These figures emphasize the components of the detector which are central to the analysis objectives of this thesis.

\subsubsection{Time Projection Chamber}

The central component of the TPC/Two-Gamma detector is the first large-scale time projection chamber (TPC) ever built [21]. The TPC gives detailed tracking information as well as values of ionization energy loss, $\mathrm{d} E / \mathrm{d} x$, for charged particles. The simultaneous measurements of momentum and $\mathrm{d} E / \mathrm{d} x$ provide a means of particle identification.

\section{Tracking}

The TPC volume is defined by concentric cylinders, with an inner radius of $20 \mathrm{~cm}$, an outer radius of $100 \mathrm{~cm}$, and a length of $200 \mathrm{~cm}$, coaxial with the beamline and centered on the interaction point. Within this volume is a gas ionization medium that consists of a mixture of 0.8 argon and 0.2 methane by volume at a pressure of 8.6 bar. Throughout the volume of the TPC run very uniform electric and 


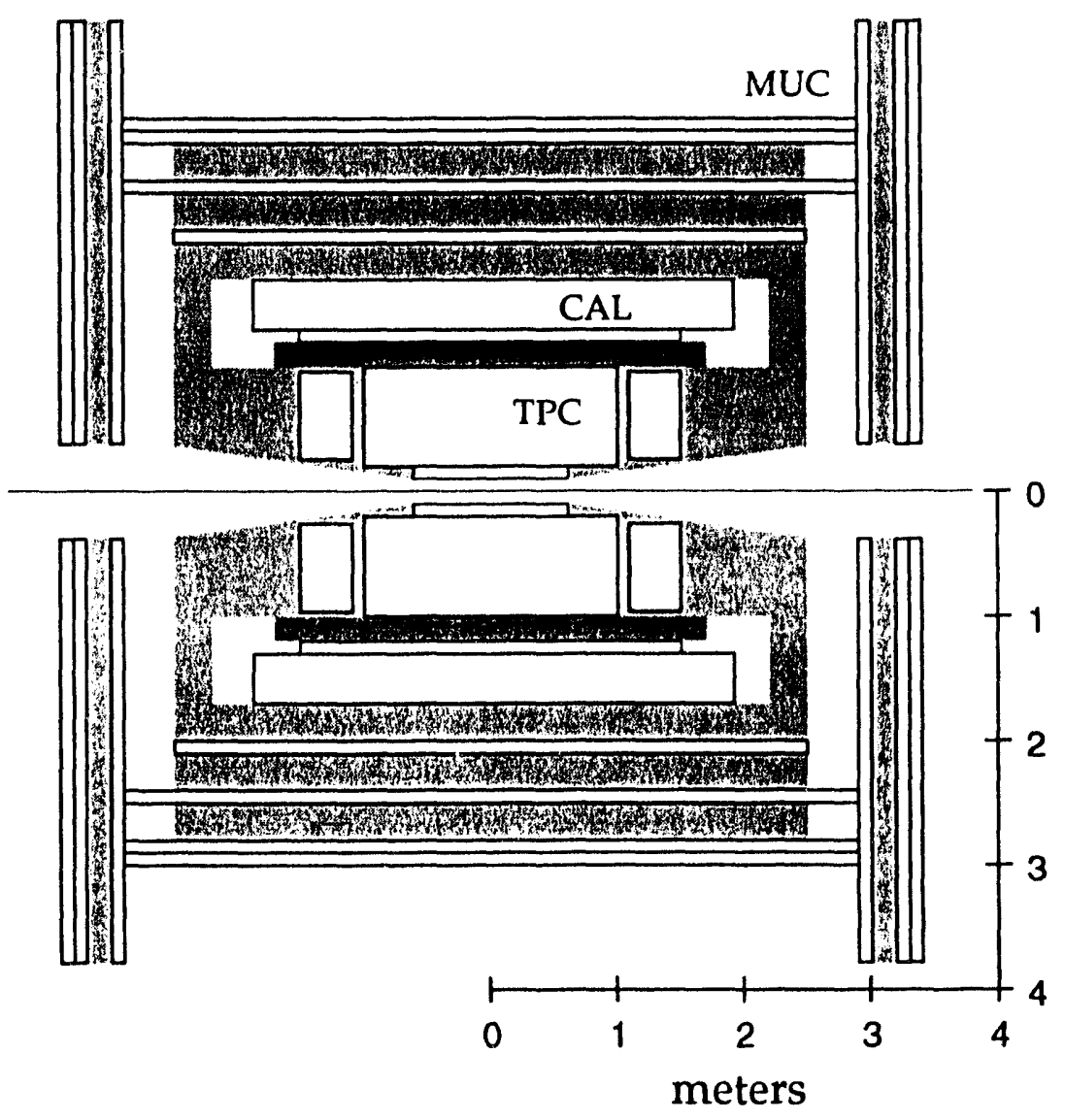

Figure 3.2: TPC/Two-Gamma detector cross-sectional side view. The geometry of the detector components is roughly cylindrical about the beamline.

magnetic fields, both parallel to the beam direction. The magnetic field bends the trajectory of a charged particle to enable a determination of its momentum, while the electric field drifts its track of ionization to sense wire and pad arrays located at the end planes. The electric field is configured by a series of grounded field wires at the end planes and a central mesh of tungsten wire held at $-55 \mathrm{kV}$. Along the walls of the cylinders is a field cage, consisting of a grid of conducting rings connected by precision resistors that maintain the uniformity and directionality of the field. The magnetic field is generated by a coil that surrounds the TPC circumference. The flux return is through a system of iron that also gives structural support to detector components located outside the TPC, and serves as the first layer of absorber for the muon detection system. Between the 1982-1983 and 19841986 experimental run periods, a conventional $4.0 \mathrm{kG}$ magnet was replaced with a $13.25 \mathrm{kG}$ superconducting magnet. The data collected during these periods are referred to as the low-field and high-field data sets, respectively. 


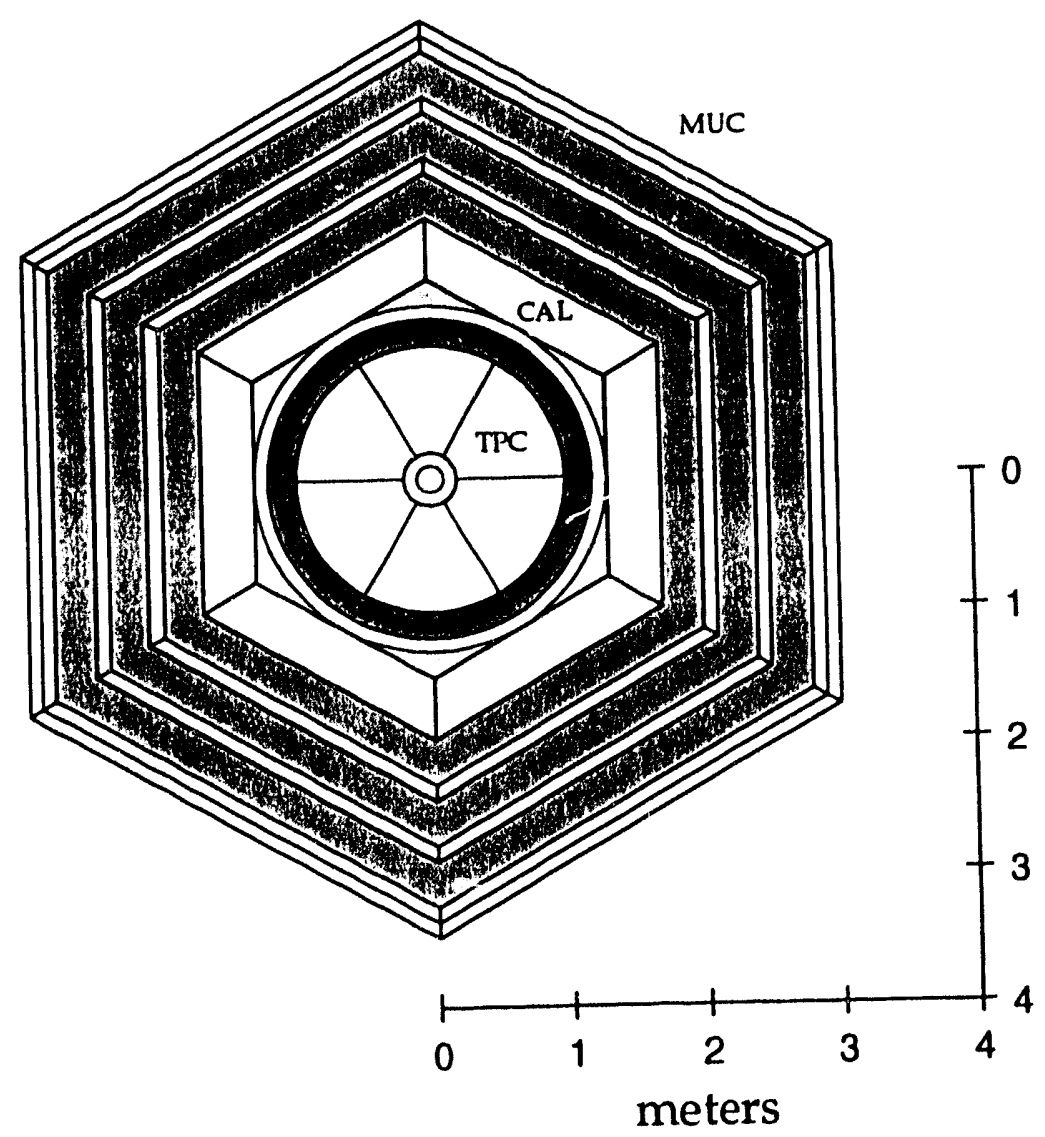

Figure 3.3: TPC/Two-Gamma detector cross-sectional end view of the central region.

When the charged particles of an event pass through the TPC volume, they leave tracks of ionization electrons from their electromagnetic interactions with the gas molecules. These electrons quickly accelerate in the electric field to an asymptotic drift velocity of $3.3 \mathrm{~cm} / \mu \mathrm{s}$. In this manner, the three-dimensional image of the ionization tracks is time-projected onto the detection planes. The radial and azimuthal positions of the ions are preserved during the drift and their original longitudinal positions are reconstructed from their drift times.

At the end planes, the tracks of ionization generate signals on arrays of sense wires and pads. Each plane is divided into six sectors, each of which covers $60^{\circ}$ in azimuth and contains 183 sense wires and 15 rows of pads, as shown in Figure 3.4. The sense wires are $20 \mu \mathrm{m}$ in diameter and run perpendicular to a radial line drawn from the beamline through the center axis of the sector. The digitized signals from the wires and pads are the raw data from which the tracks are reconstructed. 


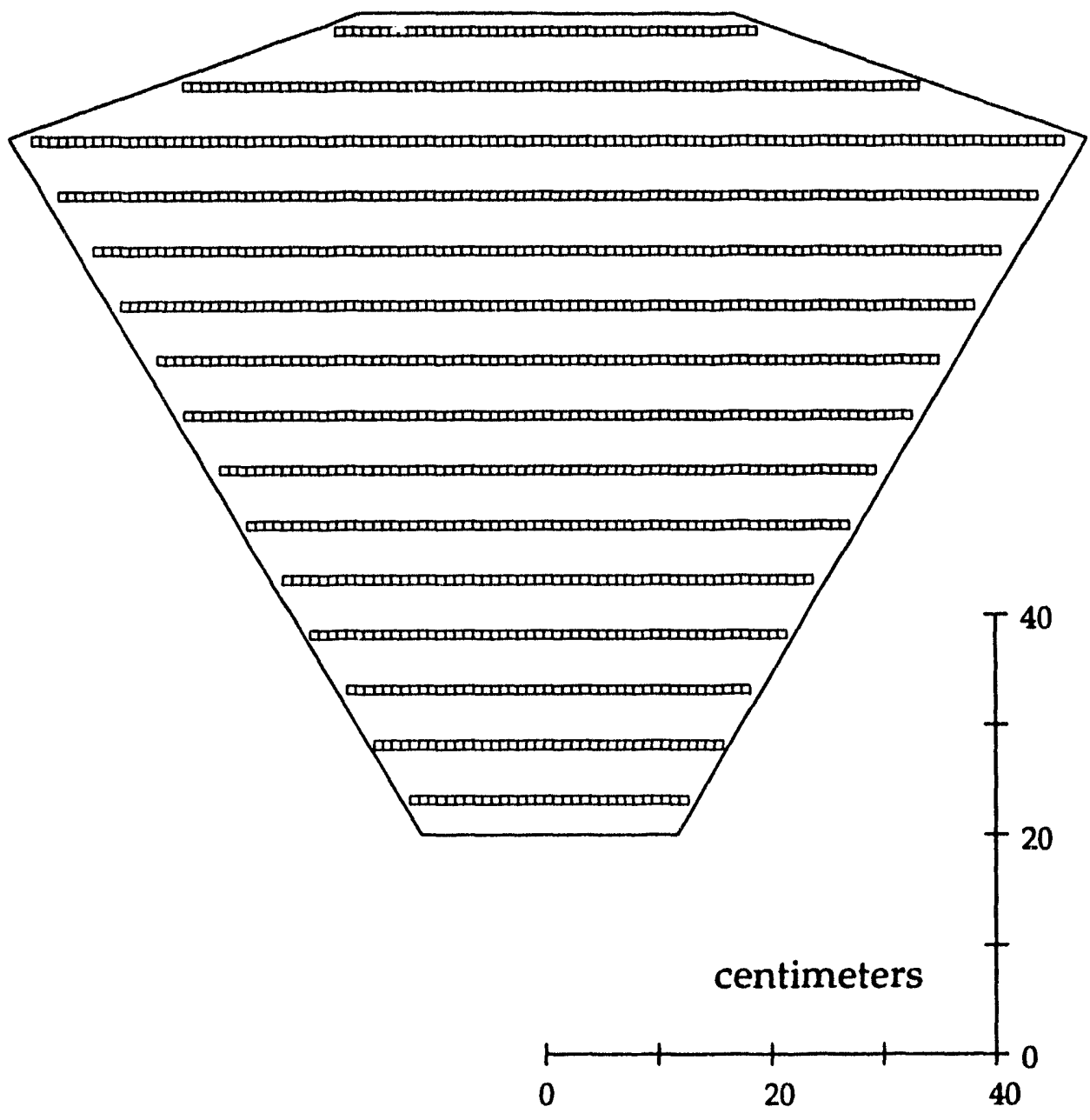

Figure 3.4: Time projection chamber sector. There are fifteen rows of pads at varying distances from the beamline. Above the pads are 183 sense wires that run parallel to the pad rows and provide $\mathrm{d} E / \mathrm{d} x$ samples.

\section{Particle Identification}

The particle identification capability of the TPC is based on simultaneous measurements of momentum and ionization energy loss, or $\mathrm{d} E / \mathrm{d} x$, of the charged particles passing through the detector volume. The track curvature depends on the momentum, while the ionization is a function of the track velocity. From these, one can determine the mass, and therefore the identity, of the charged particle.

An example of $\mathrm{d} E / \mathrm{d} x$ vs momentum values for a sample of charged tracks from $\tau^{+} \tau^{-}$candidate events is given in Figure 3.5. The curves show the expected mean values for $\mathrm{d} E / \mathrm{d} x$ as a function of momentum for the long-lived charged particle species. The distributions of $\mathrm{d} E / \mathrm{d} x$ values are known to be Gaussian out to three standard deviations, with a well-understood resolution that is a function of the 


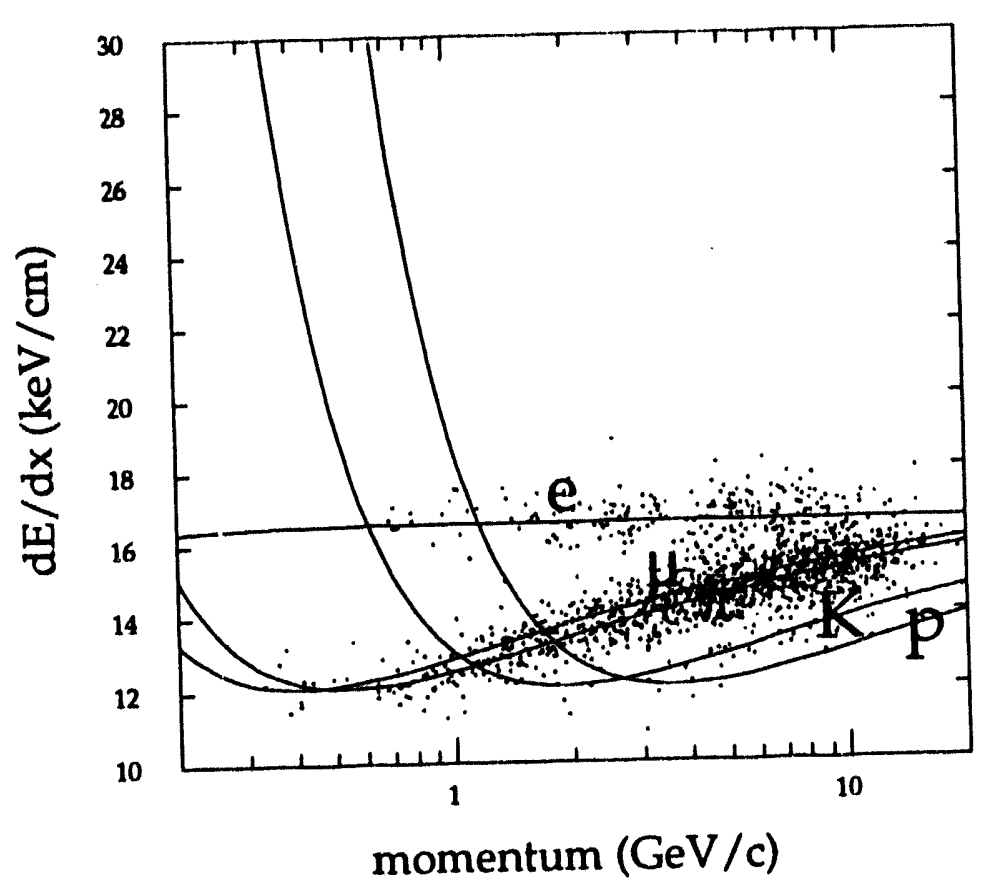

Figure 3.5: $\mathrm{d} E / \mathrm{d} x$ vs momentum for a sample of charged tracks in $\tau^{+} \tau^{-}$candidate events. The curves show the expected mean $\mathrm{d} E / \mathrm{d} x$ values for the various charged particle species as a function of momentum.

track angle and number of wire samples [22].

\subsubsection{Electromagnetic Calorimeters}

Outside the charged particle tracking system are the electromagnetic calorimeters that detect high energy photons. The calorimeters consist of alternating layers of dense material and gas-filled regions. High energy photons interact with the intense electric fields of the nuclei in the dense layers to produce $e^{+} e^{-}$pairs. The $e^{+}$and $e^{-}$then interact with the dense material to produce additional photons, which then produce more $e^{+} e^{-}$pairs. The charged particles of this electromagnetic shower generate signals on arrays of sense wires located within the gas layers of the calorimeters. The shower continues until the energies of the particles fall below the threshold for the creation of additional particles.

The TPC/Two-Gamma calorimetry is divided into central and forward regions. The central calorimeter is a group of six modules that forms a hexagonal array about the beam axis [23]. The forward calorimeters are round modules, positioned at the ends of the TPC [24]. Although the forward calorimeters play an important role in the detection of low angle $e^{+} e^{-} \rightarrow e^{+} e^{-}$events that are used in the calculation of luminosity, the analysis described in this thesis uses only the central calorimeters for charged particle identification and for detection of photons. 


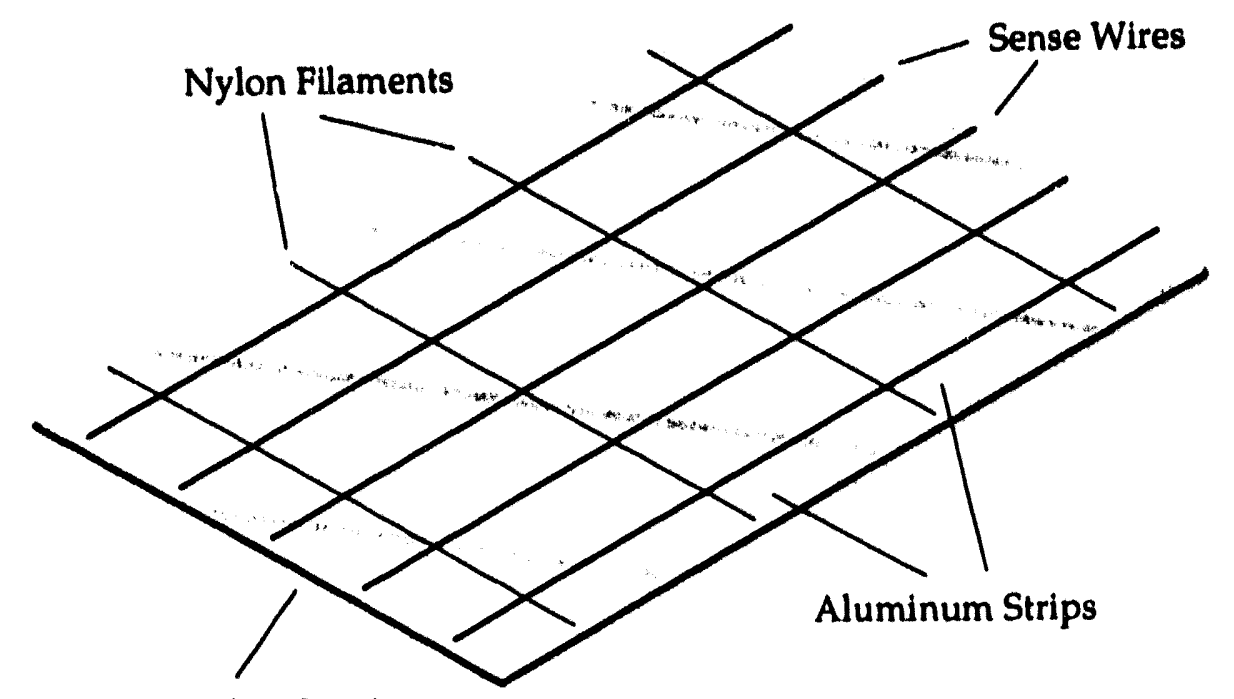

Lead/Fiberglass Laminate

Figure 3.6: Calorimeter laminate and wire mesh. The surfaces of the lead-fiberglass laminates are covered with aluminum strips oriented $+60^{\circ}$ and $-60^{\circ}$ relative to the direction of the sense wires. This configuration provides three views for the reconstruction of clusters.

The central calorimeter (CAL), shown in Figure 3.2 and Figure 3.3, consists of an array of six modules. Each module is a stack of forty layers of rectangular laminates interspersed with ionization sampling regions. Each of the laminates is composed of $1.4 \mathrm{~mm}$ lead, which serves as the electromagnetic shower medium, sandwiched between two $0.8 \mathrm{~mm}$ fiberglass sheets that provide structural support and electrical insulation. Finally, the fiberglass is covered by a conductive layer of $0.1 \mathrm{~mm}$ aluminum.

Between successive laminates are $6.0 \mathrm{~mm}$ gas-filled sampling regions, which contain the arrays of sense wires, as shown in Figure 3.6. The sense wires, which are maintained at a potential of $+1400 \mathrm{~V}$, are parallel to the beamline and are spaced at $5 \mathrm{~mm}$ intervals. Nylon filaments, which run perpendicular to the wires at $10 \mathrm{~mm}$ intervals, are woven together with the sense wires to isolate the avalanches of charged particles near the sense wires and form a grid of individual Geiger cells. The aluminum surfaces above and below the cells are grooved to form a series of conductive strips which run at $+60^{\circ}$ and $-60^{\circ}$ relative to the direction of the sense wires. This configuration provides three views for the reconstruction of the showers.

As well as being the primary detector of high energy photons, the calorimeters are also useful for charged particle identification. Figure 3.7 plots the shower energy, estimated from the calorimeters, versus the momentum, measured in the TPC, for the charged particles in a sample of $\tau^{+} \tau^{-}$candidate events. The relatively light electrons initiate electromagnetic showers, where the number of cell 


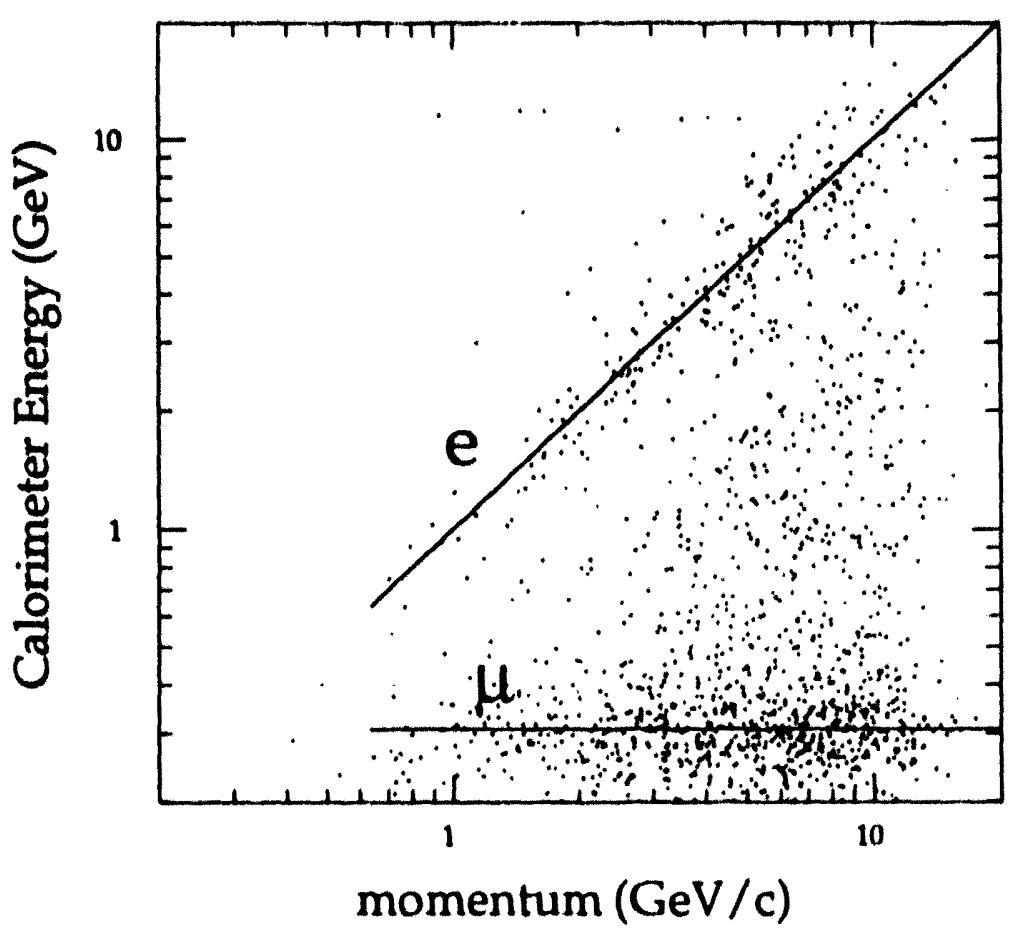

Figure 3.7: Calorimeter energy vs momentum for a sample of charged tracks in $\tau^{+} \tau^{-}$ candidate events. Electrons and positrons, which shower in the calorimeter, have energy/momentum ratios near one, while the calorimeter measurement of energy for other charged particles is independent of their momenta. The points between the electron and muon bands are due to hadronic interactions of pions and kaons.

hits increases roughly proportionally to their incident energies. For electrons the shower energy, estimated from the number of cell hits, is comparable to the momentum measured in the TPC. Other charged particle species typically generate a signal in only one or two cells per layer, and their shower energies are roughly independent of momentum. However, because of the possibility of hadronic interactions, pions and kaons may deposit additional energy in the calorimeter. Thus, the distribution of pions and kaons in Figure 3.7 extends into the region between the electron and muon bands.

\subsubsection{Muon Chambers}

The muon detection system consists of regions of iron absorber followed by layers of muon chambers (MUC) [25]. There are four hexagonal arrays of these muon chambers surrounding the central region of the detector and three layers covering each of the forward regions, as shown in Figure 3.2 and Figure 3.3. The iron absorber serves as a medium for nuclear interactions for hadrons. Muons, which very rarely generate electromagnetic showers at PEP energies and do not interact 


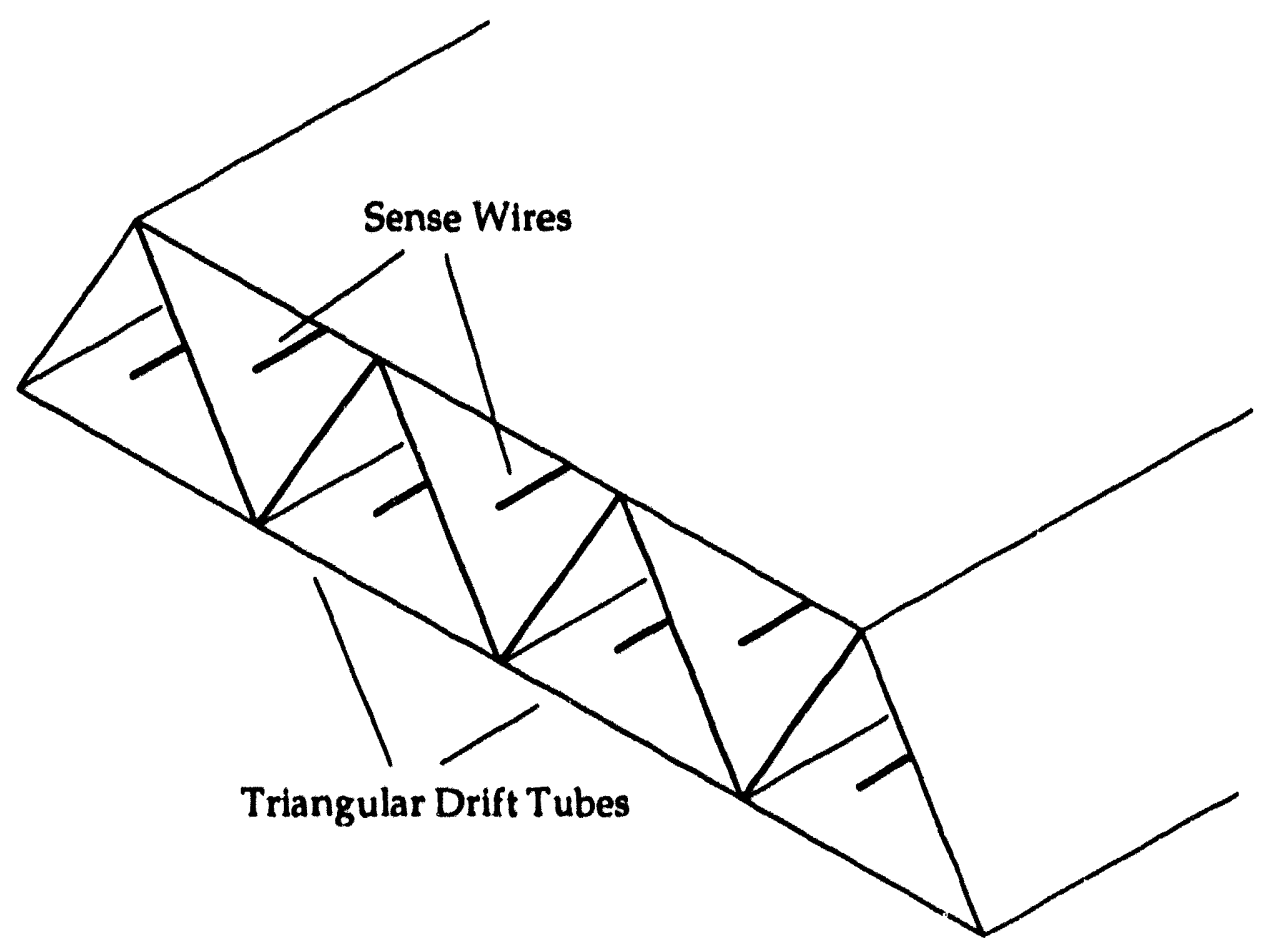

Figure 3.8: Muon chamber array. The triangular chambers are aluminum with a central tungsten sense wire.

strongly, preferentially pass through the iron layers and generate signals in the muon chambers.

Each layer of muon chambers consists of a series of close-packed triangular drift tubes, shown in Figure 3.8. The drift tubes are $0.3 \mathrm{~cm}$ thick extruded aluminum and their cross sections are equilateral triangles, $8.4 \mathrm{~cm}$ on each side. This geometry provides excellent rigidity and minimizes gaps in coverage. A gold-plated tungsten sense wire runs through the center of each drift tube. The efficiency for detection of a charged muon passing a single layer of the muon chambers is $99.5 \%$ for momentum greater than $2 \mathrm{GeV} / c$ [26]. For each event, the hits in the muon chambers are associated with the tracks observed in the TPC to determine which are to be considered muon candidates.

\subsection{Data Acquisition and Analysis}

During the experimental runs of the TPC/Two.Gamma detector, a trigger electronics system [27] determines when an event of interest has occurred. If the trigger logic is satisfied, the raw data from the various detector components are digitized and recorded on magnetic tape. These digital event records are then processed in order to reconstruct the momenta and positions of the particles in an event. In 
order to assure data quality, a portion of events are passed through a preliminary analysis during running. Later when the final calibrations have been established, all of the events are passed through the full analysis sequence.

\subsubsection{Trigger}

Since the trigger logic determines which physical events will be recorded, it is essentially the initial step in the process of event selection. The configuration of $e^{+}$and $e^{-}$bunches in the PEP collider produces crossings every $2.4 \mu \mathrm{s}$ at the interaction point. Within this time, the trigger electronics decides whether an event of interest has occurred.

In order to maximize the efficiency for identifying and recording events of interest, there are several individual trigger definitions. An event is recorded if it satisfies any of the following requirements.

- Charged Trigger. The event includes at least two charged tracks that point toward the interaction region and are separated by $30^{\circ}$ or more.

- One Charged Plus Neutral Trigger. The event includes at least one charged track and a minimum of $1 \mathrm{GeV}$ neutral energy in the calorimeters.

- Neutral Trigger. The event includes a minimum of $2 \mathrm{GeV}$ neutral energy in the calorimeters.

In addition to the trigger definitions given above, there is also a Bhabha trigger which requires a minimum of $3 \mathrm{GeV}$ neutral energy in each of the forward calorimeters. However, this trigger is used to monitor the luminosity. The acquisition rate for these common events is scaled down.

\subsubsection{Event Reconstruction}

Given the set of individual wire and pad hits in the TPC, the analysis software reconstructs the trajectories of the charged particles of an event. These tracks are then extrapolated through the layers of the calorimeters and muon chambers to predict the positions of possible hits in those detector components.

The calorimeter analysis software [28] uses the pattern of hits to determine a set of clusters. Clusters that are near the predicted positions for extrapolated charged tracks are associated with those tracks, while the remaining clusters are considered photon candidates. The shower energy of each cluster is calculated from the estimated number of Geiger cells that have fired in the cluster.

Similarly, the muon identification software associates hils in the layers of muon chambers with the extrapolated positions of the charged tracks observed in the 


\begin{tabular}{crr}
\hline Event & Cross Section (pb) & Number \\
\hline$e^{+} e^{-} \rightarrow e^{+} e^{-}$ & 9565.5 & $1,340,000$ \\
$e^{+} e^{-} \rightarrow \mu^{+} \mu^{-}$ & 165.5 & 23,200 \\
$e^{+} e^{-} \rightarrow \tau^{+} \tau^{-}$ & 136.1 & 19,100 \\
$e^{+} e^{-} \rightarrow q \bar{q}$ & 530.0 & 74,200 \\
\hline
\end{tabular}

Table 3.1: Total event estimates for the TPC/Two-Gamma experiment. For $c^{+} e^{-} \rightarrow e^{+} e^{-}$, the cross section and number of events are limited to the region at least $25^{\circ}$ from the beamline.

TPC. The pattern of associated hits is then available for the determination of muon candidates.

\subsubsection{Integrated Luminosity}

During the low-field (1982-1983) and high-field (1984-1986) experimental runs, the TPC/Two-Gamma detector collected data sets corresponding to integrated luminosities of $77.0 \mathrm{pb}^{-1}$ and $63.0 \mathrm{pb}^{-1}$, respectively. These luminosity estimates are based on the number of observed $e^{+} e^{-} \rightarrow e^{+} e^{-}$events, since this process has a well-known cross section and leaves a distinctive signature within the tracking and calorimetry of the detector. A redundant check comes from counting $q \bar{q}$ events. Table 3.1 gives estimates of the numbers of different types of events that are expected to have occurred during the runs of the TPC/Two-Gamma experiment. 


\section{Chapter 4}

\section{Measurement of $\tau^{-} \rightarrow \nu_{\tau} K_{1}^{-}$ Branching Fractions}

Decays of $\tau^{+} \tau^{-}$lepton pairs produced at $e^{+} e^{-}$colliders provide an exceptionally clean environment for studies of the resonant structure of hadronic systems below the $\tau^{-}$mass. Through the interaction of the $W^{-}$, the $\tau^{-}$decays to $\nu_{\tau}$ and a pair of quarks, either $\bar{u} d$ or $\bar{u} s$, which then undergo hadronization and strong decay processes to produce long-lived mesons, predominantly $\pi$ and $K$. The wcll-measured hadronic decays of the $\tau^{-}$lepton are observed to proceed through coupling of the $W^{-}$to $\bar{u} d$ and $\bar{u} s$ resonant states. The $(\pi \pi)^{-}$and $(K \pi)^{-}$decays are dominated by the $\rho^{-}$and $K^{*-}$ vector channels $[16]$, while the $(\pi \pi \pi)^{-}$decays proceed through the $a_{1}^{-}$axial-vector channel [17]. However, the resonance structure of the $(K \pi \pi)^{-}$ decays has remained an open question due to their small branching fractions and the difficulty of identifying or reconstructing the strange mesons. The obvious candidates for $(K \pi \pi)^{-}$resonances are the $K_{1}^{-}(1270)$ and $K_{1}^{-}(1400)$ [29], which are mixtures of the $\bar{u}$ s analogs of the $a_{1}^{-}$and $b_{1}^{-}$.

This chapter describes an analysis of the resonance structure of the decay $\tau^{-} \rightarrow \nu_{r}(K \pi \pi)^{-}$through the three-charged mode, $\tau^{-} \rightarrow \nu_{r} K^{-} \pi^{+} \pi^{-*}$. We find evidence that these decays do in fact proceed through the $K_{1}^{-}$channels, and report measurements of the branching fractions for $\tau^{-} \rightarrow \nu_{\tau} K_{1}^{-}(1270)$ and $\tau^{-} \rightarrow$ $\nu_{\tau} K_{1}^{-}(1400)$. The magnitude of these branching fractions provides information concerning coupling of the weak axial-vector $\bar{u} s$ current, while the ratio of these branching fractions is relevant in the determination of the mixing angle of the two $K_{1}$ states [30].

\subsection{The Method}

This analysis is based entirely on charged track information from the time projection chamber (TPC), and utilizes its excellent tracking and particle identification 


\begin{tabular}{|c|c|c|c|c|}
\hline & \multicolumn{2}{|c|}{$K_{1}(1270)$} & \multicolumn{2}{|c|}{$K_{1}(1400)$} \\
\hline mass (MeV) & 1270 & \pm 10 & 1402 & \pm 7 \\
\hline width (MeV) & 90 & \pm 20 & 174 & \pm 13 \\
\hline \multirow[t]{2}{*}{ decay modes } & \multicolumn{2}{|c|}{ fractions (\%) } & \multicolumn{2}{|c|}{ fractions (\%) } \\
\hline & total & $K^{-} \pi^{+} \pi^{-}$ & total & $K^{-} \pi^{+} \pi^{-}$ \\
\hline$K^{*} \pi$ & $16 \pm 5$ & $7 \pm 2$ & $94 \pm 6$ & $42 \pm 3$ \\
\hline$K_{0}^{*}(1430) \pi$ & $28 \pm 4$ & $12 \pm 2$ & & \\
\hline$K \rho$ & $42 \pm 6$ & $14 \pm 2$ & $3 \pm 3$ & $1 \pm 1$ \\
\hline$K \omega$ & $11 \pm 2$ & $10 \pm 2$ & $1 \pm 1$ & $1 \pm 1$ \\
\hline$K f_{0}(1400)$ & $3 \pm 2$ & $2 \pm 2$ & $2 \pm 2$ & $1 \pm 1$ \\
\hline total & 100 & 45 & 100 & 45 \\
\hline
\end{tabular}

Table 4.1: Properties of the $K_{1}^{-}$resonances. For each of the decay modes of the $K_{1}^{-}(1270)$ and $K_{1}^{-}(1400)$, the table lists both the total branching fraction and the branching fraction to the three-charged $K^{-} \pi^{+} \pi^{-}$final state, calculated from isospin symmetry and known branching fractions of the intermediate resonances.

capabilities. In order to obtain a sample of $\tau^{-} \rightarrow \nu_{r} K^{-} \pi^{+} \pi^{-}$events, we first select for $\tau^{+} \tau^{-}$events in the $\tau_{1+3}$ topology, where the numbers in the subscript give the numbers of charged particles in each of the $\tau$ decays. Then the three-prong tracks of these events are each classified as $\pi^{ \pm}$or $K^{ \pm}$candidates. Since the number of $\tau^{-} \rightarrow \nu_{r} K^{-} \pi^{+} \pi^{-}$events is quite small, the priorities of the selection process are to minimize background and to allow efficient $\pi^{ \pm} / K^{ \pm}$separation for the three-prong tracks of the sample.

We determine the resonance structure of the $\mathrm{K}^{-} \pi^{+} \pi^{-}$decays of the $\tau^{-}$lepton by observing the distribution of events in the three-dimensional invariant mass space defined by $m_{k-\pi^{+} \pi^{-}}^{2}, m_{k-\pi^{+}}^{2}$, and $m_{\pi^{+} \pi_{-}}^{2}$. This choice of axes provides sensitivity to the presence of resonant states in the charged particle combinations $K^{-} \pi^{+} \pi^{-}, K^{-} \pi^{+}$, and $\pi^{+} \pi^{-}$. The $K^{-} \pi^{+} \pi^{-}$system may proceed through one or both of the $K_{1}^{-}$states, while the $K^{-} \pi^{+}$and $\pi^{+} \pi^{-}$combinations are likely candidates for resonant states such as $K^{*}$ and $\rho$. For each hypothetical channel for the $\mathrm{K}^{-} \pi^{+} \pi^{-}$decay of the $\tau^{-}$, we derive an analytical expression for the expected distribution of events in the invariant mass space described above. We then use an extended maximum likelihood fit to determine the most probable resonant composition of the $\tau^{-} \rightarrow \nu_{\tau} K^{-} \pi^{+} \pi^{-}$decay.

The mass, width, and decay properties of the $K_{j}^{-}$states, which are expected to dominate this decay, are listed in Table 4.1. Unlike the $\bar{u} d$ axial-vector analogs $a_{1}^{-}$and $b_{1}^{-}$, which decay almost exclusively to $(\rho \pi)^{-}$and $(\omega \pi)^{-}$respectively, the $\bar{u} s$ $K_{1}^{-}$states both have rather complicated sets of decay modes that lead to different 


\begin{tabular}{|c|c|c|c|c|}
\hline \multirow{2}{*}{$\begin{array}{l}\text { Decay Mode } \\
K^{*} \pi\end{array}$} & Intermediate & \multicolumn{2}{|c|}{ Final State } & \multirow{2}{*}{$\begin{array}{r}\text { Fraction } \\
44 \%\end{array}$} \\
\hline & $K^{00} \pi^{-} \quad\left(\frac{2}{3}\right)$ & $K^{-} \pi^{+} \pi^{-}$ & $\left(\frac{2}{3}\right)$ & \\
\hline & & $\bar{K}^{0} \pi^{0} \pi^{-}$ & $\left(\frac{1}{3}\right)$ & $22 \%$ \\
\hline & $K^{*-} \pi^{0}$ & $\bar{K}^{0} \pi^{-} \pi^{0}$ & $\left(\frac{2}{3}\right)$ & $22 \%$ \\
\hline & & $K^{-} \pi^{0} \pi^{0}$ & $\left(\frac{1}{3}\right)$ & $11 \%$ \\
\hline \multirow{4}{*}{$K_{0}^{*}(1430) \pi$} & $\bar{K}_{0}^{* 0}(1430) \pi^{-}$ & $K^{-} \pi^{+} \pi^{-}$ & $\left(\frac{2}{3}\right)$ & $44 \%$ \\
\hline & & $\bar{K}^{0} \pi^{0} \pi^{-}$ & $\left(\frac{1}{2}\right)$ & $22 \%$ \\
\hline & $K_{0}^{*-}(1430) \pi^{0}\left(\frac{1}{3}\right)$ & $K^{0} \pi^{-} \pi^{0}$ & $\left(\frac{2}{3}\right)$ & $22 \%$ \\
\hline & & $K^{-} \pi^{0} \pi^{0}$ & $\left(\frac{1}{3}\right)$ & $11 \%$ \\
\hline \multirow[t]{2}{*}{$\overline{K \rho}$} & $K^{-} \rho^{0}$ & $K^{-} \pi^{+} \pi^{-}$ & (1) & $33 \%$ \\
\hline & $\bar{K}^{0} \rho^{-}$ & $K^{0} \pi^{-} \pi^{0}$ & (1) & $67 \%$ \\
\hline \multirow[t]{3}{*}{$K \omega$} & $K-\omega$ & $K^{-} \pi^{+} \pi^{-} \pi^{0}$ & $(89 \%)$ & $89 \%$ \\
\hline & & $K^{-} \pi^{+} \pi^{-}$ & $(2 \%)$ & $2 \%$ \\
\hline & & $K^{-} \pi^{0} \gamma$ & $(9 \%)$ & $9 \%$ \\
\hline \multirow[t]{4}{*}{$K f_{0}(1400)$} & $K-f_{0}(1400)$ & $K^{-} \pi^{+} \pi^{-}$ & $(93 \%)\left(\frac{2}{3}\right)$ & $62 \%$ \\
\hline & & $K^{-} \pi^{0} \pi^{0}$ & $(93 \%)\left(\frac{1}{3}\right)$ & $31 \%$ \\
\hline & & $K^{-} K^{+} K^{-}$ & $(7 \%)\left(\frac{1}{2}\right)$ & $3 \%$ \\
\hline & & $K^{-} K^{0} \bar{K}^{0}$ & $(7 \%)\left(\frac{1}{2}\right)$ & $3 \%$ \\
\hline
\end{tabular}

Table 4.2: Decay modes of the $K_{1}^{-}$resonances. The fractions for the intermediate and final states are calculated from isospin symmetry and known branching fractions. Net fractions for the final state $K^{-} \pi^{+} \pi^{-}$are listed in bold.

groups of final state mesons. All of the established decays of the two $K_{1}^{-}$states occur through intermediate resonant channels, which provide an additional handle in identifying contributions from the $K_{1}^{-}(1270)$ and $K_{1}^{-}(1400)$. The $K_{1}^{-}(1270)$ has significant decays to $K^{*} \pi, K_{0}^{*}(1430) \pi, K \rho$, and $K \omega$, while the $K_{1}^{-}(1400)$ decays almost entirely to $K^{*} \pi$. Note that the decays to $K_{0}^{*}(1430) \pi$ and $K^{\prime} f_{0}(1400)$ are through broad resonances with central masses greater or equal to those of the $K_{1}^{-}$ states. In addition to the overall $K_{1}^{-}$branching fractions, Table 4.1 also lists the contributions from each of the decay modes to the three-charged $K^{-} \pi^{+} \pi^{-}$final state. The values for the three-charged final state contributions are calculated from the known branching fractions of the intermediate resonant states or from isospin symmetry, as listed in Table 4.2. Remarkably, the net contribution to the three-charged final state, $K^{-} \pi^{+} \pi^{-}$, is about $45 \%$ for both the $K_{1}^{-}(1270)$ and the $K_{1}^{-}(1400)$. 


\subsection{Event Selection}

The data sample used in this analysis includes both the low-field (1982-1983) and high-field (1984-1986) experimental runs. The total sample has an integrated luminosity of $140 \mathrm{pb}^{-1}$ at an $e^{+} e^{-}$center of mass energy of $29 \mathrm{GeV}$. The event selection is based entirely on kinematic and particle identification information from the charged tracks observed in the TPC. We select events in the $\tau_{1+3}$ topology.

- Each event must have a total of 4 reconstructed tracks with a net charge of zero. This requirement excludes most of the $q \bar{q}$ events, which tend to have higher charged multiplicity. It also excludes $e^{+} e^{-}$and $\mu^{+} \mu^{-*}$ events, although the radiative analogs, $e^{+} e^{-\gamma}$ and $\mu^{+} \mu^{-} \gamma$, may still pass if the photon converts to an $e^{+} e^{-}$pair. Note that this cut also excludes those $\tau_{1+3}$ events in which photons from $\pi^{0}$ decay convert to produce additional $e^{+} e^{-}$tracks.

- Each of these tracks must have momentum greater than $0.3 \mathrm{GeV} / \mathrm{c}$, have an angle relative to the beamline of at least $30^{\circ}$, and extrapolate to the interior of an imaginary cylinder $5 \mathrm{~cm}$ in radius and $10 \mathrm{~cm}$ in length, coaxial with the beamline and centered at the nominal interaction point.

- One of the tracks must be at least $140^{\circ}$ and less than $178^{\circ}$ from each of the other three tracks. This defines the one-prong and three-prong candidate tracks of the $\tau_{1+3}$ topology.

- The invariant mass of the three-prong tracks, assuming $\pi$ masses, must be less than $2.0 \mathrm{GeV} / c^{2}$. This is another strict cut against $q \bar{q}$ backgrounds.

- The invariant mass of all the reconstructed tracks, assuming $\pi$ masses, must be at least $3.0 \mathrm{GeV} / \mathrm{c}^{2}$. This cut removes most of the two-photon events.

- The $\mathrm{d} E / \mathrm{d} x$ of each of the three-prong tracks must satisfy $\boldsymbol{\chi}_{\pi}^{2}<\boldsymbol{9 . 0}$ or $\chi_{K}^{2}<9.0$, where $\chi^{2}$ is the goodness of fit of the measured $\mathrm{d} E / \mathrm{d} x$ and momentum to the expected $\mathrm{d} E / \mathrm{d} x$ vs momentum curve for a given particle species. Here we require that the three-prong tracks be hadrons, as expected for $\tau^{-}$decays with charged multiplicities greater than one. This requirement excludes $e^{+} e^{-} \gamma$ and $\mu^{+} \mu^{-} \gamma$ events where photon conversion to $e^{+} e^{-}$fakes the $\tau_{1+3}$ topology.

- Each of the three-prong candidates with momentum greater than $2.0 \mathrm{GeV} / \mathrm{c}$ must have at least 80 wire $\mathrm{d} E / \mathrm{d} x$ samples. This ensures the level of resolution necessary to make an efficient separation of $\pi^{ \pm}$and $K^{ \pm}$. 


\begin{tabular}{lccc}
\hline \multicolumn{4}{c}{ Event Selection Eficiency (\%) } \\
Decay Mode & Low-Field & High-Field & Average \\
\hline$\nu_{\tau} \pi^{-} \pi^{+} \pi^{-}$ & 10.7 & 16.5 & 13.3 \\
$\nu_{\tau} K^{-} \pi^{+} \pi^{-}$ & 9.6 & 15.0 & 12.0 \\
$\nu_{\tau} K^{-} K^{+} \pi^{-}$ & 7.1 & 12.4 & 9.5 \\
$\nu_{\tau} K^{-} K^{+} K^{-}$ & 2.4 & 5.6 & 3.9 \\
\hline$\nu_{\tau} \pi^{-} \pi^{+} \pi^{-} \pi^{0}$ & 7.5 & 12.1 & 9.6 \\
$\nu_{\tau} K^{-} \pi^{+} \pi^{-} \pi^{0}$ & 6.7 & 11.2 & 8.7 \\
$\nu_{\tau} K^{-} K^{+} \pi^{-} \pi^{0}$ & 4.6 & 9.1 & 6.6 \\
$\nu_{\tau} K^{-} K^{+} K^{-} \pi^{0}$ & 1.4 & 3.9 & 2.5 \\
\hline
\end{tabular}

Table 4.3: Selection efficiencies, estimated from Monte Carlo, for three-prong events averaged over the various one-prong decays. Note the lower efficiencies for decays with $K^{ \pm}$or with $\pi^{0}$.

- The scalar sum of momentum for all charged tracks must be greater than 4.5 GeV/c and less than 24.0 GeV/c. This requirement removes essentially all of the residual two-photon events (at the low end) and $e^{+} e^{-} \gamma$ and $\mu^{+} \mu^{-} \gamma$ events (at the high end).

This $\tau_{1+3}$ sample consists of 518 events with an estimated purity of $99.1 \%$. Backgrounds estimates, based on Monte Carlo simulation, are $3.5 q \bar{q}$ and $1.3 \tau_{1+1}$ events with negligible contributions from $e^{+} e^{-}(\gamma), \mu^{+} \mu^{-}(\gamma)$, and two-photon events.

Selection efficiencies for various three-prong decays of the $\tau^{-}$lepton are estimated from Monte Carlo simulations, as listed in Table 4.3. Note that as the number of $K^{ \pm}$in the decay increases, the selection efficiency decreases. The addition of $\pi^{0}$ to the final state also lowers the selection efficiency. For example, compared with the $\pi^{-} \pi^{+} \pi^{-}$decay, the $K^{-} \pi^{+} \pi^{-}$decay has a relative selection efficiency of about $90 \%$ and the $\pi^{-} \pi^{+} \pi^{-} \pi^{0}$ decay about $75 \%$. There are two sources for these lower selection efficiencies. First, as the net mass of the decay particles for a particular mode increases, the relative velocities of these particles are more restricted and result in a more collimated decay. If the tracks are too close together, the TPC is unable to provide independent wire $\mathrm{d} E / \mathrm{d} x$ measurements for each of the tracks. These events are then excluded due to inadequate $\pi^{ \pm} / K^{ \pm}$resolution. The selection efficiencies for decays with $\pi^{0}$ are also decreased since the $\pi^{0}$ decays to photons which may then produce conversion $e^{+} e^{-}$tracks. These events then fail the charged track multiplicity criterion.

\subsection{Charged Particle Identification}




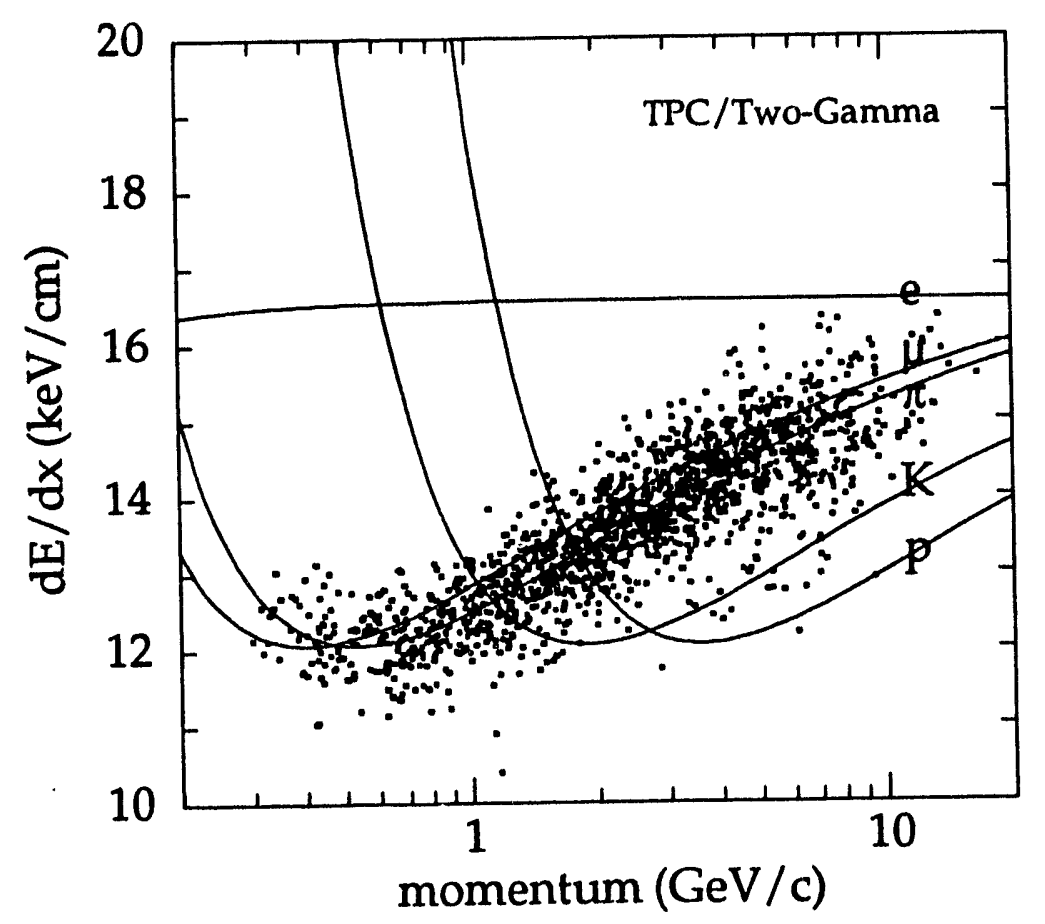

Figure 4.1: $\mathrm{d} E / \mathrm{d} x$ vs momentum for the three-prong tracks of the $\tau_{1+3}$ event sample, along with the expected curves for the various particle species.

The three-prong tracks of the $\tau_{1+3}$ sample are expected to consist entirely of $\pi^{ \pm}$ and $K^{ \pm}$. The separation of these tracks into $\pi^{ \pm}$and $K^{ \pm}$candidates is based on momentum and $\mathrm{d} E / \mathrm{d} x$ measurements from the TPC. Figure 4.1 plots $\mathrm{d} E / \mathrm{d} x$ versus momentum for these tracks and gives a rough idea of the relative abundance of $\pi^{ \pm}$and $K^{ \pm}$in the sample. While most of the tracks are distributed about the mean value curve of the $\pi^{ \pm}$, there is also a small but unmistakable population of tracks distributed about the $K^{ \pm}$curve. The $\mathrm{d} E / \mathrm{d} x$ distributions for charged particle species are known to be Gaussian out to three standard deviations with a well-understood resolution that is a function of the angle and number of wire samples [22]. Our requirement of at least 80 wire $\mathrm{d} E / \mathrm{d} x$ samples gives an average $\mathrm{d} E / \mathrm{d} x$ resolution of $3.2 \%$ and an average $\pi^{ \pm} / K^{ \pm}$separation of over three sigma for the momentum region above $2.0 \mathrm{GeV} / c$. Tracks below $2.0 \mathrm{GeV} / c$ are considered to be $\pi^{ \pm}$, since Monte Carlo simulations predict that fewer than $4 \%$ of the $K^{ \pm}$from three-prong $\tau^{-}$decays will have such low momenta. This is simply a consequence of the high mass of the $K^{ \pm}$relative to that of the $\tau^{-}$, which limits their relative velocity.

Protons from background sources pose a particular concern since they would likely be misidentified as $K^{ \pm}$due to their low $\mathrm{d} E / \mathrm{d} x$ values through the high momentum region. The sources of possible proton contamination are $q \bar{q}$ events of unusually low charged multiplicity and nuclear interactions of particles with the 


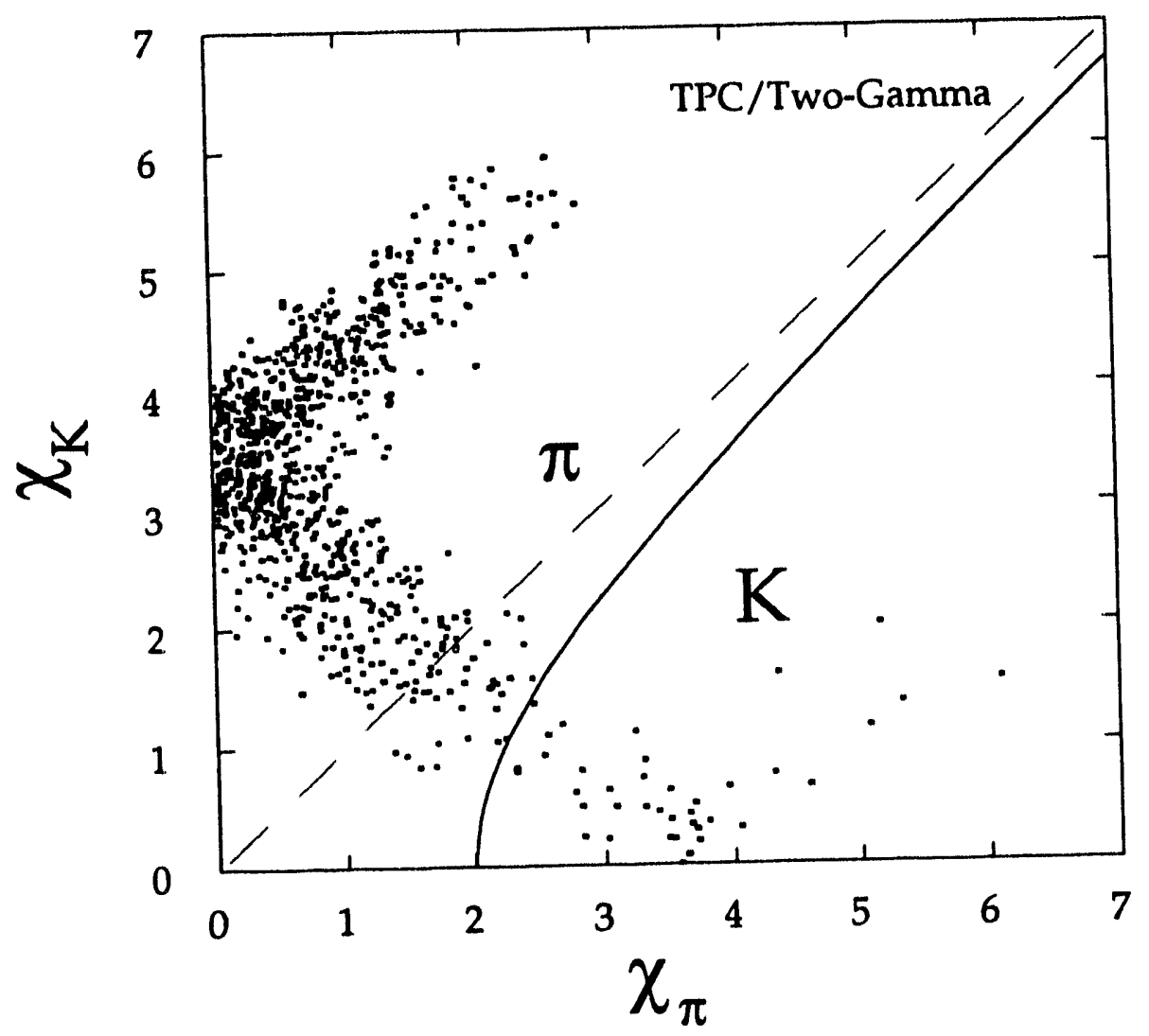

Figure 4.2: $\chi_{K}$ vs $\chi_{\pi}$ for the three-prong tracks of the $\tau_{1+3}$ sample. The solid line $\chi_{\pi}^{2}-\chi_{K}^{2}=4$ separates the tracks into $\pi^{ \pm}$and $K^{ \pm}$candidates.

beampipe or inner pressure wall of the TPC. The selection criteria were chosen to be especially efficient in excluding $q \bar{q}$ events from the $\tau^{+} \tau^{-}$sample. Monte Carlo simulations predict less than one proton in the $\tau_{1+3}$ sample from $q \bar{q}$ background. Interactions with the ordinary bulk matter of the detector would produce protons and not anti-protons. Of the tracks in the region below the $K^{ \pm}$mean, there are six positive and ten negative tracks. Therefore, there is no indication of a significant number of protons within the sample.

A plot of the $\chi_{K}$ vs $\chi_{\pi}$ values for the three-prong tracks with momentum greater than $2.0 \mathrm{GeV} / c$ is shown in Figure 4.2 , where $\chi \equiv \sqrt{\chi^{2}}$. Since the $\mathrm{d} E / \mathrm{d} x$ resolutions of the tracks vary little through this momentum region and the distance between the expected $\mathrm{d} E / \mathrm{d} x$ values for $\pi^{ \pm}$and $K^{ \pm}$is roughly constant, the tracks fall into three bands depending on whether their $\mathrm{d} E / \mathrm{d} x$ value is above the $\pi^{ \pm}$curve, between the $\pi^{ \pm}$and $K^{ \pm}$curves, or below the $K^{ \pm}$curve. These three regions are described approximately by the equations,

$$
\begin{aligned}
& \chi_{K}=\chi_{\pi}+c \\
& \chi_{K}+\chi_{\pi}=c
\end{aligned}
$$




\begin{tabular}{cc}
\hline Decay Class & Population \\
\hline$\nu_{\tau} \pi^{-} \pi^{+} \pi^{-} \geq 0$ neutral & 484 \\
$\nu_{\tau} K^{-} \pi^{+} \pi^{-} \geq 0$ neutral & 23 \\
$\nu_{\tau} \pi^{-} K^{+} \pi^{-} \geq 0$ neutral & 7 \\
$\nu_{\tau} K^{-} K^{+} \pi^{-} \geq 0$ neutral & 4 \\
$\nu_{\tau} K^{-} \pi^{+} K^{-} \geq 0$ neutral & 0 \\
$\nu_{\tau} K^{-} K^{+} K^{-} \geq 0$ neutral & 0 \\
\hline
\end{tabular}

Table 4.4: Populations for the $\tau^{-}$three-prong decays, categorized according to the charged particle classification.

$$
\chi_{K}=\chi_{\pi}-c
$$

where $c$ is the separation of $\pi^{ \pm}$and $K^{ \pm}$curves in number of standard deviations. The distribution in Figure 4.2 shows that the typical $\pi^{ \pm} / K^{ \pm}$separation for these tracks is about 3.5 sigma.

We use the $\chi_{\pi}$ and $\chi_{K}$ values to separate the three-prong tracks into $\pi^{ \pm}$and $K^{ \pm}$candidates. The probability of a track being $\pi^{ \pm}$or $K^{ \pm}$is given by,

$$
\begin{aligned}
P_{\pi} & \propto f_{\pi} e^{-\chi_{\pi}^{2} / 2} \\
P_{K} & \propto f_{K} e^{-\chi_{K}^{2} / 2}
\end{aligned}
$$

where $f_{\pi}$ and $f_{K}$ are the particle fractions for $\pi^{ \pm}$and $K^{ \pm}$for the given momentum of the track. The relative probability is then,

$$
\left(P_{K} / P_{\pi}\right)=\left(f_{K} / f_{\pi}\right) e^{-\left(x_{K}^{2}-\chi_{\pi}^{2}\right) / 2}
$$

and contours of constant relative probability are given by hyperbolic curves of the form,

$$
\chi_{\pi}^{2}-\chi_{K}^{2}=a
$$

where $a$ is a constant. Since the fraction of $K^{ \pm}$in the sample is small, we select the value $a=4$, given by the solid line in Figure 4.2, to divide the tracks into $\pi^{ \pm}$ and $K^{ \pm}$candidates.

With the tracks separated into $\pi^{ \pm}$and $K^{ \pm}$candidates, we classify the events according to the possible combinations of charged particles. These populations are given in Table 4.4. To examine the resonance structure of the decay $\tau^{-} \rightarrow \nu_{\tau} K^{-} \pi^{+} \pi^{-}$, we use the $23 \mathrm{~K}^{-} \pi^{+} \pi^{-}$candidates. The background events in the $\mathrm{K}^{-} \pi^{+} \pi^{-}$sample are expected to be primarily due to $\pi^{-} \pi^{+} \pi^{-}$events where one of the $\pi^{-}$is misidentified as $K^{-}$. From the assumption of Gaussian $\mathrm{d} E / \mathrm{d} x$ distributions for charged particles and the $\pi^{ \pm} / K^{ \pm}$classification criterion $\chi_{\pi}^{2}-\chi_{K}^{2}=4$, we calculate 

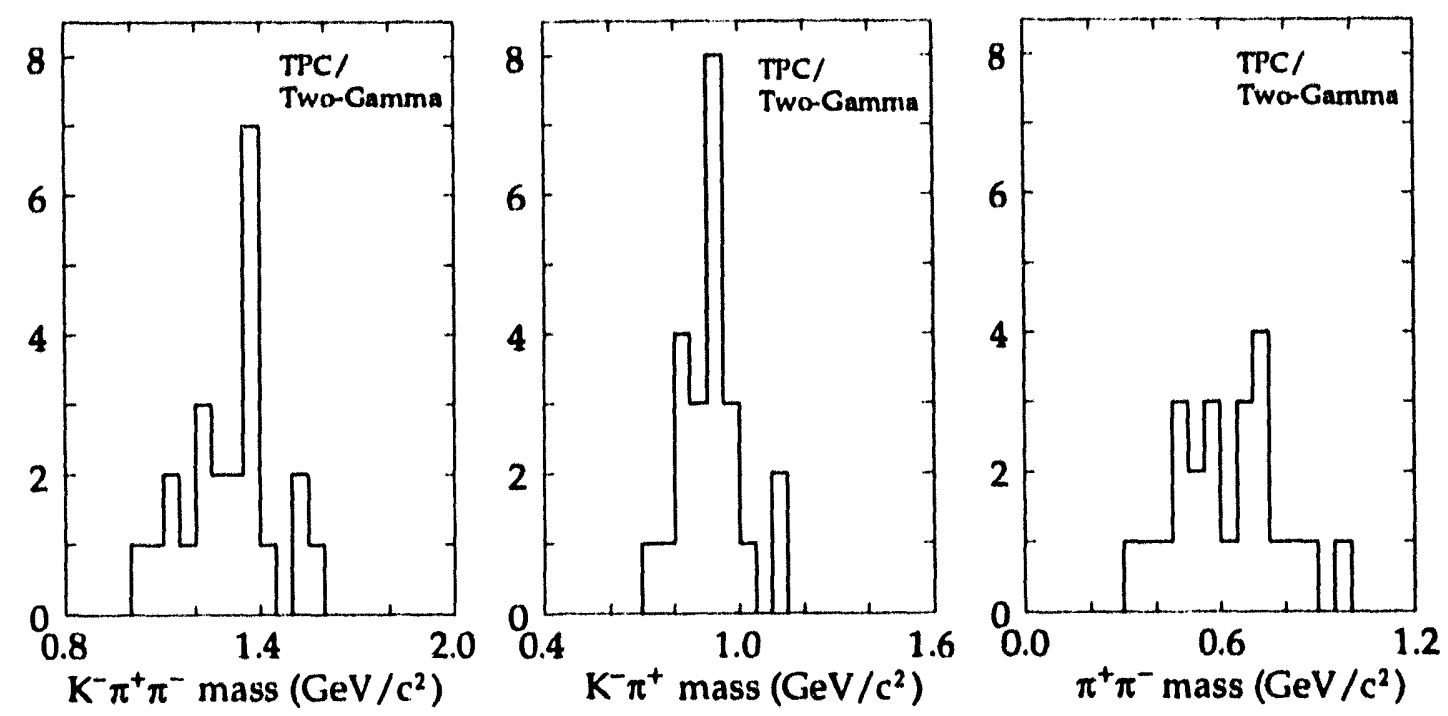

Figure 4.3: Invariant mass distributions of the $23 \mathrm{~K}^{-} \pi^{+} \pi^{-}$candidate events of the $\tau_{1+3}$ sample.

the $\pi^{-} \rightarrow K^{-}$misidentification probability for each of the $\pi^{-}$of the $\pi^{-} \pi^{+} \pi^{-}$candidates. The sum of these misidentification probabilities provides a background estimate of $6.3 \pm 2.7$ events, where we have included a $15 \%$ systematic error due to uncertainties in $\mathrm{d} E / \mathrm{d} x$ parameterization.

The $K^{-} \pi^{+} \pi^{-}, K^{-} \pi^{+}$, and $\pi^{+} \pi^{-}$invariant mass plots for the $K^{-} \pi^{+} \pi^{-}$candidate events are shown in Figure 4.3. For the $m_{K-\pi+\pi-}$ plot, most of the events are clustered in the region between $1.2 \mathrm{GeV} / c^{2}$ and $1.4 \mathrm{GeV} / c^{2}$, giving a narrower distribution than would be expected for non-resonant phase space decay channels. The $m_{K-\pi^{+}}$spectrum shows a distinct $K^{*}$ peak, consistent with a large contribution from the $K_{1}^{-}(1400)$, which decays almost entirely through the $K^{* 0}$. Finally, the $m_{\pi^{+} \pi^{-}}$plot gives only a hint of $\rho$ signal, which would be a signature for the $K_{1}^{-}(1270)$.

\subsection{Neutral Particle Identification}

The selection of the $\mathrm{K}^{-} \pi^{+} \pi^{-}$candidate event sample described in the preceeding sections is based entirely on charged particle information. In order to check for evidence of neutral particles, particularly $\pi^{0}$, we look for energetic photons that are associated with the three-prong $\tau$ decays of the $\mathrm{K}^{-} \pi^{+} \pi^{-}$sample. At PEP energies, the decay products are well-separated into distinct hemispheres and most photons are easily associated with one decay or the other, as shown in Figure 5.4. For each event, we define a decay axis as the sum of the momenta of the three-prong system of charged particles, and include photons within $45^{\circ}$ of this axis that have energies 
of at least $0.4 \mathrm{GeV}$. Photon pairs with an invariant mass between $0.07 \mathrm{GeV} / \mathrm{c}^{2}$ and $0.20 \mathrm{GeV} / c^{2}$ are considered to be $\pi^{0}$ candidates. Using these criteria, we find no $\pi^{0}$ candidates among the $23 \mathrm{~K}^{-} \pi^{+} \pi^{-}$candidate events, although there are 6 events with 1 energetic photon and 1 event with 2 energetic photons.

In order to estimate the number of $\mathrm{K}^{-} \pi^{+} \pi^{-}$candidate events with an additional $\pi^{0}$, we use Monte Carlo simulations of the decays $r^{-} \rightarrow \nu_{\tau} K^{-} \pi^{+} \pi^{-}$and $\tau^{-} \rightarrow \nu_{\tau} K^{-} \pi^{+} \pi^{-} \pi^{0}$. The simulated events are passed through the analysis software to obtain estimates of the expected fractions of events that will be observed to have a reconstructed $\pi^{0}$ or high energy photons. A maximum likelihood fit of the Monte Carlo predictions to the number of reconstructed $\pi^{0}$ observed in the data estimates $0 \pm 2$ events with an additional $\pi^{0}$, while a similar fit based on the number of energetic photons estimates $7 \pm 4$. The discrepancy in the two estimates gives an idea of the systematic uncertainties in the Monte Carlo simulation of the electromagnetic calorimeter. However, these estimates do provide rough limits on the number of events with $\pi^{0}$. We expect that the Monte Carlo simulation may overestimate the $\pi^{0}$ reconstruction efficiency, and almost certainly underestimates the number of fake photons from hadronic interactions of $\pi$ and $K$ in the calorimeter. Consequently the actual number of events with $\pi^{0}$ is likely somewhere between the two values given.

From the branching fractions and selection efficiencies for $\tau^{-} \rightarrow \nu_{\tau} \pi^{-} \pi^{+} \pi^{-}$and $\tau^{-} \rightarrow \nu_{\tau} \pi^{-} \pi^{+} \pi^{-} \pi^{0}[8]$, we expect that about $40 \%$ of the background events will have an additional $\pi^{0}$. This would contribute $2.5 \pm 1.1$ events with $\pi^{0}$. Also note that the decay $K_{1}^{-} \rightarrow K^{-} \omega$ results in a final state of $K^{-} \pi^{+} \pi^{-} \pi^{0}$. After adjusting for relative selection efficiencies of the various decay modes, we expect that $16.4 \%$ of $K_{1}^{-}(1270)$ events and $1.4 \%$ of $K_{1}^{-}(1400)$ events will also include a $\pi^{0}$.

Although we find no evidence for a significant number of $\mathrm{K}^{-} \pi^{+} \pi^{-} \pi^{0}$ events in our sample, the relatively low $\pi^{0}$ reconstruction efficiency prevents us from ruling out their presence. The question of $\mathrm{K}^{-} \pi^{+} \pi^{-} \pi^{0}$ contribution must ultimately be resolved by experiments with both charged particle identification and efficient $\pi^{0}$ reconstruction.

\subsection{Decay Models}

In order to determine the resonant contributions of the $K_{1}^{-}(1270), K_{1}^{-}(1400)$, and other possible channels for the decay $\tau^{-} \rightarrow \nu_{\tau} K^{-} \pi^{+} \pi^{-}$, we use Monte Carlo methods to obtain analytical expressions that model the expected distributions of these decay events in the three-dimensional invariant mass space defined by $\mathrm{m}_{\mathrm{K}^{-} \pi^{+} \pi^{-}}$, $m_{K^{-} \pi^{+}}^{2}$, and $m_{\pi^{+} \pi^{-}}^{2}$.

The decay modeling begins with the derivation of the Lorentz invariant phase space for $\tau^{-} \rightarrow \nu_{\tau} K^{-} \pi^{+} \pi^{-}$. The calculation is simplified by factorization [8], which 


\begin{tabular}{llllllll}
\hline & Decay & \multicolumn{7}{c}{$K^{-}$Model Parameters } \\
\hline & Mode & Mass & Width & Mass & Width & Mass & Width \\
\hline$K_{1}^{-}(1270)$ & $K^{*} \pi$ & 1.262 & 0.102 & 0.898 & 0.059 & & \\
& $K_{0}^{*}(1430) \pi$ & 1.264 & 0.087 & 1.306 & 0.238 & & \\
& $K \rho$ & 1.263 & 0.094 & & & 0.761 & 0.102 \\
& $K \omega$ & 0.933 & 0.144 & & & 0.573 & 0.108 \\
& $K f_{0}(1400)$ & 1.264 & 0.093 & & & 0.861 & 0.313 \\
\hline$K_{1}^{-}(1400)$ & $K^{*} \pi$ & 1.373 & 0.199 & 0.898 & 0.061 & & \\
& $K \rho$ & 1.404 & 0.120 & & & 0.768 & 0.083 \\
& $K \omega$ & 0.980 & 0.345 & & & 0.537 & 0.252 \\
& $K f_{0}(1400)$ & 1.400 & 0.162 & & & 0.820 & 0.255 \\
\hline
\end{tabular}

Table 4.5: Model parameters for the $K_{1}^{-}$resonances. These effective masses and widths are used to describe the expected distribution of events in the $\mathrm{K}^{-} \pi^{+} \pi^{-}, \mathrm{K}^{-} \pi^{+}, \pi^{+} \pi^{-}$ invariant mass space.

allows the isolation of the hadronic portion of the phase space according to

$$
d \phi\left[\tau^{-} \rightarrow \nu_{\tau} K^{-} \pi^{+} \pi^{-}\right]=d \phi\left[\tau^{-} \rightarrow \nu_{\tau} Q\right] \cdot d \phi\left[Q \rightarrow K^{-} \pi^{+} \pi^{-}\right] \cdot d m_{K \pi \pi}^{2},
$$

where $Q$ represents the $K^{-} \pi^{+} \pi^{-}$system. The phase space for the initial two-body decay is given by

$$
d \phi\left[\tau^{-} \rightarrow \nu_{\tau} Q\right]=\frac{1}{2^{9} \pi^{6}} \frac{\left(m_{\tau}^{2}-m_{K \pi \pi}^{2}\right)}{m_{\tau}^{2}} d \Omega .
$$

Here the factor $\left(m_{\tau}^{2}-m_{K \pi \pi}^{2}\right)$ is proportional to the momentum of the $K^{-} \pi^{+} \pi^{-}$ system in the rest frame of the $\tau^{-}$. The phase space for the three-body hadronic system may be expressed in Dalitz form as,

$$
d \phi\left[Q \rightarrow K^{-} \pi^{+} \pi^{-}\right]=\frac{1}{2^{11} \pi^{7}} \frac{1}{m_{K \pi \pi}^{2}} d m_{K \pi}^{2} d m_{\pi \pi}^{2} .
$$

For the decay $\tau^{-} \rightarrow \nu_{\tau} K^{-} \pi^{+} \pi^{-}$, the overall phase space probability density function is then given by

$$
d \phi\left[\tau^{-} \rightarrow \nu_{\tau} K^{-} \pi^{+} \pi^{-}\right]=\frac{1}{2^{20} \pi^{13}} \frac{1}{m_{\tau}^{2}} d \Omega\left(\frac{m_{\tau}^{2}}{m_{K \pi \pi}^{2}}-1\right) d m_{K \pi \pi}^{2} d m_{K \pi}^{2} d m_{\pi \pi}^{2} .
$$

Note how the expression approaches zero as the invariant mass of the $\mathrm{K}^{-} \pi^{+} \pi^{-}$ system approaches the $\tau$ mass, the boundary for the accessible phase space. 
This phase space expression is used as the basis for the analytical models that we use to describe the invariant mass distribution of events for the various hypothetical channels of the $\tau^{-} \rightarrow \nu_{\tau} K^{-} \pi^{+} \pi^{-}$decay. Our basic probability density function is then,

$$
f\left(m_{K \pi \pi}, m_{K \pi}, m_{\pi \pi}\right)=N\left(\frac{m_{\tau}^{2}}{m_{K \pi \pi}^{2}}-1\right)
$$

where the constants and the integration over the angular orientation of the initial $\tau^{-}$have been relegated to an overall normalization factor $N$. To this we add optional Breit-Wigner functions,

$$
g(Q)=\frac{m_{Q}^{2} \Gamma_{Q}^{2}}{\left(m_{Q}^{2}-m_{0 Q}^{2}\right)^{2}+m_{0 Q}^{2} \Gamma_{Q}^{2}}
$$

to allow modeling of resonances for the $\mathrm{K}^{-} \pi^{+} \pi^{-}, \mathrm{K}^{-} \pi^{+}$and $\pi^{+} \pi^{-}$charged particle combinations, designated here by $Q$. The $m_{0 Q}$ and $\Gamma_{Q}$ parameters are the effective central mass and width values of the resonances. The inclusion of these BreitWigner functions gives the final from of the invariant mass probability density function,

$$
f\left(m_{K \pi \pi}, m_{K \pi}, m_{\pi \pi}\right)=N \cdot g(K \pi \pi) \cdot g(K \pi) \cdot g(\pi \pi) \cdot\left(\frac{m_{\tau}^{2}}{m_{k \pi \pi}^{2}}-1\right) .
$$

Not all of the Breit-Wigner functions are necessary for any given decay. For example, in modeling the decay chain $\tau^{-} \rightarrow \nu_{\tau} K_{1}^{-}(1400) / K_{1}^{-}(1400) \rightarrow K^{* 0} \pi^{-} /$ $\bar{K}^{* 0} \rightarrow K^{-} \pi^{+}$, we parameterize the Breit-Wigner functions for the $K^{-} \pi^{+} \pi^{-}$and $K^{-} \pi^{+}$systems of particles, but not for the $\pi^{+} \pi^{-}$combination.

For each of the decay modes of the $K_{1}^{-}(1270)$ and $K_{1}^{-}(1400)$, we generate events with the $\tau^{-}$generator KORALB [31], to which we have added decays to the $K_{l}^{-}$resonances. These events are then passed through a detector simulation in order to model acceptance and resolution effects. The analytical expression for the expected decay probability density is then fit to the invariant mass distribution of these Monte Carlo events. The fit determines the best values for the effective mass and width parameters of the Breit-Wigner functions, listed in Table 4.5. Note that the $K^{-} \pi^{+} \pi^{-}$mass values for the $K \omega$ decay modes of the $K_{1}^{-}(1270)$ and $K_{1}^{-}(1400)$ are low due to the additional $\pi^{0}$ in the final state of this decay. Finally, these expressions for the individual decay modes are combined, according to their relative selection efficiencies and branching fractions, as given in Table 4.6, to give net invariant mass distributions for the $K_{1}^{-}(1270)$ and the $K_{1}^{-}(1400)$. The projections of these distributions onto the $K^{-} \pi^{+} \pi^{-}, K^{-} \pi^{+}$, and $\pi^{+} \pi^{-}$axes are given in Figure 4.4.

Using the $\pi^{-} \pi^{+} \pi^{-}$candidates of the $\tau_{1+3}$ data sample, we derive a similar analytical expression for the expected invariant mass distribution for the background. For each $\pi^{-}$with momentum greater than $2.0 \mathrm{GeV} / c$, we replace the $\pi^{-}$mass 


\begin{tabular}{llrrrr}
\hline \multicolumn{5}{c}{ Normalized Decay Fractions } \\
\hline & $\begin{array}{c}\text { Decay } \\
\text { Mode }\end{array}$ & $\begin{array}{c}\text { Total } \\
\text { Fraction(\%) }\end{array}$ & $\begin{array}{r}K^{-} \pi^{+} \pi^{-} \\
\text {Fraction(\%) }\end{array}$ & $\begin{array}{c}\text { Selection } \\
\text { Efficiency(\%) }\end{array}$ & $\begin{array}{r}\text { Normalized } \\
\text { Fraction(\%) }\end{array}$ \\
\hline$K_{1}^{-}(1270)$ & $K^{*} \pi$ & $16 \pm 5$ & 44.4 & 12.6 & 17.7 \\
& $K_{0}^{*}(1430) \pi$ & $28 \pm 4$ & 44.4 & 12.3 & 30.2 \\
& $K \rho$ & $42 \pm 6$ & 33.3 & 11.4 & 31.4 \\
& $K \omega$ & $11 \pm 2$ & 91.0 & 8.3 & 16.4 \\
& $K f_{0}(1400)$ & $3 \pm 2$ & 62.0 & 11.8 & 4.3 \\
\hline$\left.K_{i}^{-}(1400)\right)$ & $K^{*} \pi$ & $94 \pm 6$ & 44.4 & 12.9 & 94.0 \\
& $K \rho$ & $3 \pm 3$ & 33.3 & 11.7 & 2.0 \\
& $K \omega$ & $1 \pm 1$ & 91.0 & 8.6 & 1.4 \\
& $K f_{0}(1400)$ & $2 \pm 2$ & 62.0 & 12.0 & 2.6 \\
\hline
\end{tabular}

Table 4.6: Normalized decay fractions for $K_{1}^{-} \rightarrow K^{-} \pi^{+} \pi^{-}$. The total fractions are the measured branching fractions for the $K_{1}^{-}(1270)$ and $K_{1}^{-}(1400)$. The normalized fractions take into account the fraction of each decay to the $\mathrm{K}^{-} \pi^{+} \pi^{-}$final state and the selection efficiencies. These normalized fractions are the coefficients used in the linear combination of individual decays of the $K_{1}^{-}(1270)$ and $K_{1}^{-}(1400)$.

\begin{tabular}{cccc}
\hline Decay & $\begin{array}{c}\text { Population } \\
\text { Likelihood }\end{array}$ & $\begin{array}{c}\text { Efficiency } \\
\epsilon K_{1} / \epsilon_{3}\end{array}$ & $\begin{array}{c}\text { Branching Fraction (\%) } \\
\text { TPC } / 2 \gamma\end{array}$ \\
\hline$\nu_{\tau} K_{1}^{-}(1270)$ & $5.4 \pm 4.8$ & $0.36 \pm 0.04$ & $0.41 \pm 0.41$ \\
$\nu_{r} K_{1}^{-}(1400)$ & $11.0 \pm 5.8$ & $0.39 \pm 0.04$ & $0.76 \pm 0.40$ \\
$\nu_{\tau} K_{1}^{-}$ & $16.4 \pm_{-5.7}^{5.7}$ & $0.38 \pm 0.04$ & $1.1 \pm_{-0.34}^{0.43}$ \\
\hline
\end{tabular}

Table 4.7: Branching fractions for $T^{-} \rightarrow \nu_{\tau} K_{1}^{-}(1270), T^{-} \rightarrow \nu_{\tau} K_{1}^{-}(1400)$, and total $\tau^{-} \rightarrow \nu_{\tau} K_{1}^{-}$, assuming that the process $\tau^{-} \rightarrow \nu_{\tau} K^{-} \pi^{+} \pi^{-}$proceeds entirely through the $K_{1}^{-}$channels. The efficiency definition is described in the text.

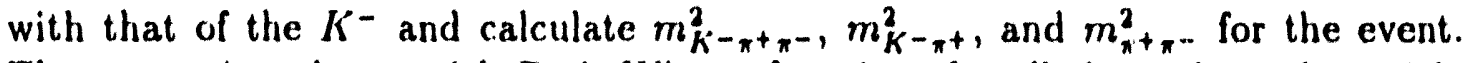
The expression above, with Breit-Wigner functions for all three charged particle combinations, is then fit to these invariant mass points. 

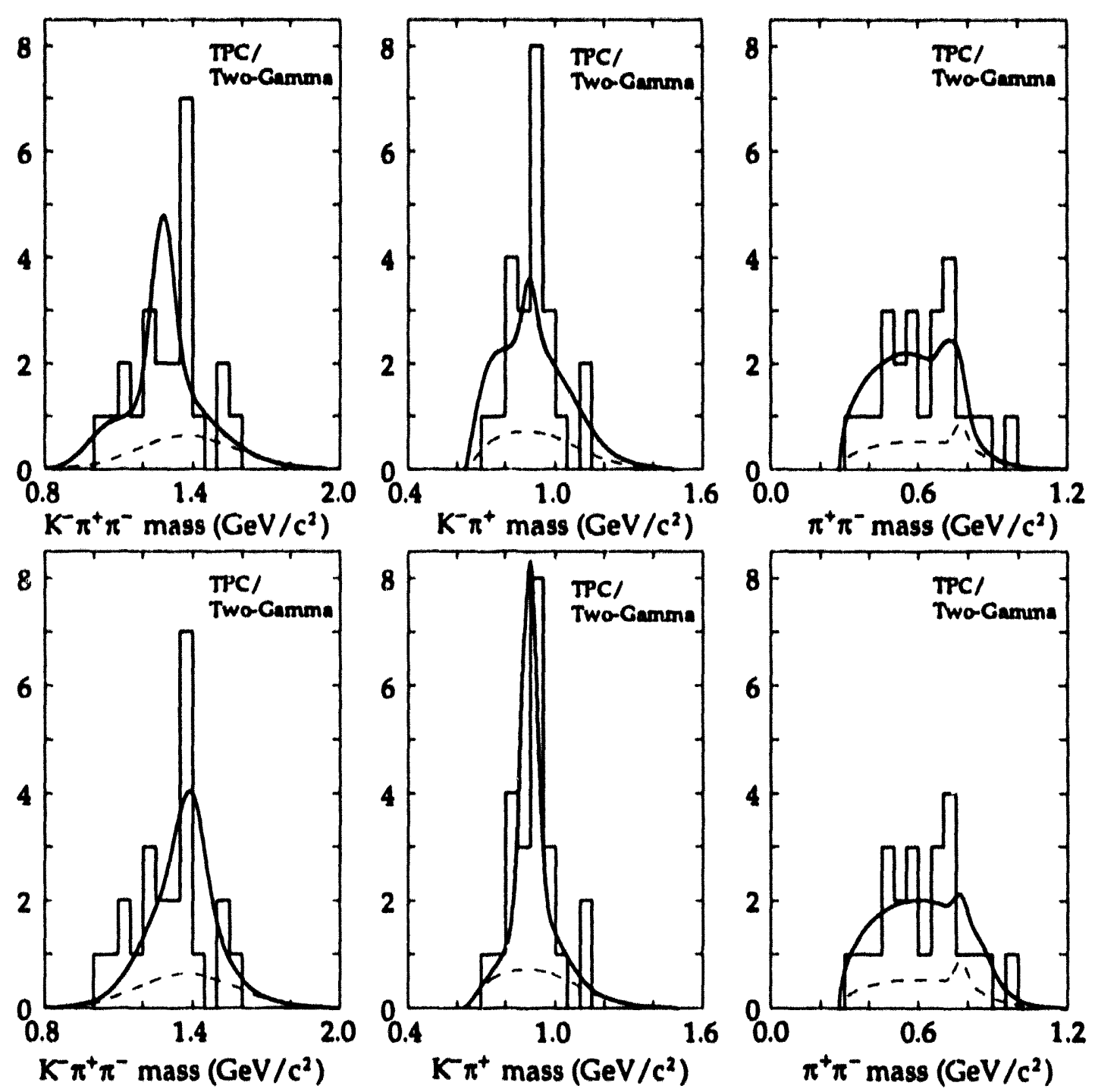

Figure 4.4: Invariant mass distributions of the $K_{1}^{-}(1270)$ and $K_{1}^{-}(1400)$ models, compared with the $K^{-} \pi^{+} \pi^{-}$candidate events. The upper plots are for the $K_{1}^{-}(1270)$ plus background, while the lower plots are for the $K_{1}^{-}(1400)$ plus background. The dashed curve shows the estimated background from misidentified $\pi^{-} \pi^{+} \pi^{-}$events.

\subsection{Branching Fractions}

Assuming that the decay $\tau^{-} \rightarrow \nu_{r} K^{-} \pi^{+} \pi^{-}$is dominated by the two $K_{1}^{-}$channels, we construct an extended maximum likelihood function,

$$
\mathcal{L}_{E}=\left(e^{-\nu} \frac{\nu^{n}}{n !}\right) \prod_{a}^{n}\left(\sum_{j} \frac{\nu_{j}}{\nu} f_{j}\left(m_{K \pi \pi}^{a}, m_{K \pi}^{a}, m_{\pi \pi}^{a}\right)\right)
$$



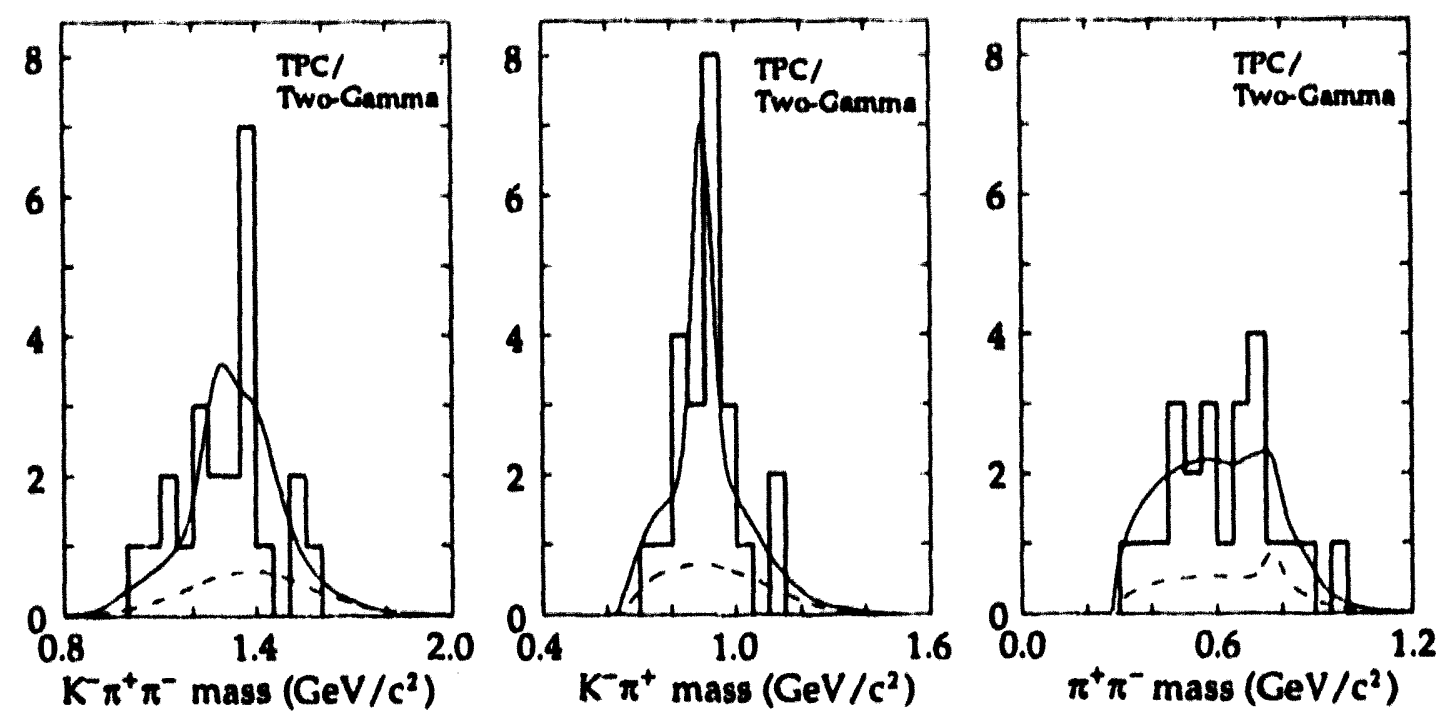

Figure 4.6: Invariant mass distributions of best fit $K_{1}^{-}(1270)$ and $K_{1}^{-}(1400)$, compared with the $\mathrm{K}^{-} \pi^{+} \pi^{-}$candidate events of the $r_{1+3}$ sample. The dashed curve shows the estimated background from misidentified $\pi^{-} \pi^{+} \pi^{-}$events, while the solid curve shows the background plus the best fit combination of $K_{1}^{-}(1270)$ and $K_{1}^{-}(1400)$.

where $a$ runs over the $\mathrm{K}^{-} \pi^{+} \pi^{-}$candidate events. The $\nu_{j}$ and $f_{j}$ are the expected populations and predicted invariant mass distributions of $K_{1}^{-}(1270), K_{1}^{-}(1400)$, and background events. The sum of the $\nu_{j}$ is given by $\nu$, and the Poisson factor accounts for statistical fluctuations in the total population of the sample. Using the background estimate of $6.3 \pm 2.7$ events, we determine the most probable values for the $K_{1}^{-}(1270), K_{1}^{-}(1400)$, and total $K_{1}^{-}$populations, listed in Table 4.7. As a consistency check, we perform the likelihood fit without using our background estimate based on misidentification probabilities. Using the likelihood fit alone, we obtain a background estimate of $8.5 \pm 7.7$ events, in good agreement with the value of $6.3 \pm 2.7$ arrived at from misidentification probabilities.

The branching fractions are calculated according to

$$
B_{K_{1}}=B_{3} \cdot\left(N_{K_{1}} / N_{3}\right) /\left(\epsilon_{K_{1}} / \epsilon_{3}\right)
$$

where $B_{3}$ is the topological branching fraction to three-prongs $14.06 \pm 0.25 \%$ [8], $N_{3}$ is the number of events in our $\tau_{1+3}$ sample, and $\epsilon_{K_{1}} / \epsilon_{3}$ is the selection efficiency for $K_{1}^{-}$events in the $\mathrm{K}^{-} \pi^{+} \pi^{-}$sample relative to that for three-prong events in the $\tau_{1+3}$ sample. We estimate a systematic error of $25 \%$ from uncertainties in $\mathrm{d} E / \mathrm{d} x$ parameterization, event selection efficiencies, and modeling of the $K_{1}^{-}(1270)$ and $K_{1}^{-}(1400)$ decays. The Monte Carlo invariant mass distributions for the best fit values of $K_{1}^{-}(1270)$ and $K_{1}^{-}(1400)$ plus background are compared with those of the $K^{-} \pi^{+} \pi^{-}$candidates in Figure 4.5, while the error contours for the $K_{i}^{-}$branching fractions are shown in Figure 4.6. Given the current branching fractions for the 


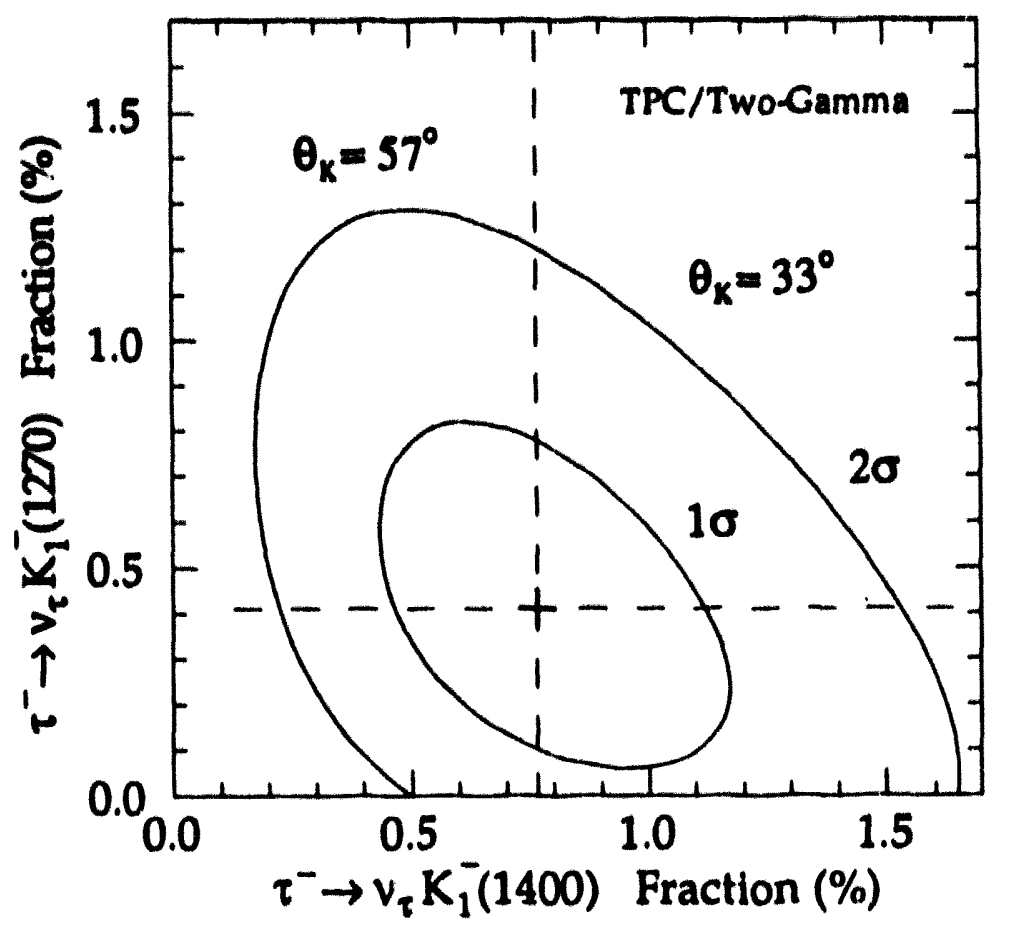

Figure 4.6: Statistical error contours for the $\tau^{-} \rightarrow \nu_{\tau} K_{1}^{-}(1270)$ and $\tau^{-} \rightarrow \nu_{\tau} K_{1}^{-}(1400)$ branching fractions. The shaded regions represent the ranges of the ratio of $K_{1}^{-}$branching fractions predicted for $K_{1}^{-}$mixing angles of $33^{\circ}$ and $57^{\circ}$ and $\mathrm{SU}(3)$ breaking of $|\delta| \leq 0.2$.

decays $K_{1}(1270) \rightarrow K^{*} \pi$ and $K_{1}(1400) \rightarrow K^{*} \pi[8]$, we use our $\tau^{-} \rightarrow \nu_{\tau} K_{1}^{-}$values to obtain

$$
B\left(\tau^{-} \rightarrow \nu_{\tau} K^{\cdot 0} \pi^{-}\right)=0.51+0.26 \% \text {, }
$$

consistent with a measurement from CLEO [32] of $0.38 \pm 0.17 \%$. The partial decay rates and masses of the $K_{1}(1270)$ and $K_{1}(1400)$ predict a mixing angle of $\theta_{K_{1}} \approx 33^{\circ}$ or $57^{\circ}[30]$. Our ratio of $K_{1}^{-}$branching fractions favors the mixing angle of $\theta_{K_{1}} \approx 33^{\circ}$ for reasonable estimates of $\mathrm{SU}(3)$ breaking, as shown by the corresponding shaded regions of Figure 4.6.

The results that we have presented 80 far are based on the assumption that the process $\tau^{-} \rightarrow l_{\tau} K^{-} \pi^{+} \pi^{-}$proceeds entirely through the $K_{1}^{-}(1270)$ and $K_{1}^{-}(1400)$ channels. In order to check for non- $K_{1}^{-}$contributions to the decay $\tau^{-} \rightarrow \nu_{\tau} K^{-} \pi^{+} \pi^{-}$, we use Monte Carlo methods to obtain expressions for the expected invariant mass distributions of non-resonant $\bar{K}^{* 0} \pi^{-}, K^{-} \rho^{0}$, and $K^{-} \pi^{+} \pi^{-}$. We perform likelihood fits where one of these non-resonant components is included along with the $K_{1}^{-}(1270)$, the $K_{1}^{-}(1400)$, and background. The most likely value for the branching fraction of $\bar{K}^{* 0} \pi^{-}$, which has an invariant mass distribution very similar to the $K_{1}^{-}(1400)$, is $0.19 \pm 0.20$, while branching fractions for the $K^{-} \rho^{0}$ and $K^{-} \pi^{+} \pi^{-}$ are $0.01 \pm 0.07$ and $0.04 \pm 0.16$. Although we are unable to rule out contributions from 


\begin{tabular}{|c|c|c|c|c|}
\hline \multicolumn{5}{|c|}{ Resonant State Branching Fractions (\%) } \\
\hline & & id states & & s states \\
\hline Pseudoscalar & $\pi^{-}$ & $11.6 \pm 0.4$ & $K^{-}$ & $0.67 \pm 0.23$ \\
\hline Vector & $\rho^{-}$ & $22.2 \pm 1.0$ & $K^{*-}$ & $1.42 \pm 0.18$ \\
\hline Axial-Vector & $a_{1}^{-}$ & $18.7 \pm 1.0$ & $K_{1}^{-}$ & $1.17 \pm 0.41$ \\
\hline
\end{tabular}

Table 4.8: $r^{-}$branching fractions for $\bar{u} d$ and $\bar{u} s$ resonant states. The ratio of our measurement of the total branching fraction for the $\bar{u} s$ axial-vector decay $\tau^{-} \rightarrow \nu_{\tau} K_{1}^{-}$ to the branching fraction for $T^{-} \rightarrow \nu_{\tau} a_{1}^{-}$is similar to the $\bar{u} s / \bar{u} d$ ratio for the vector and pseudoscalar decays.

these non-resonant channels, our overall results are consistent with $K_{1}^{-}$dominance of the $(K \pi \pi)^{-}$decays of the $\tau^{-}$lepton.

\subsection{Discussion}

This resonance analysis provides the first evidence for the strange axial-vector decay of the $\tau^{-}$lepton. The likelihood fit clearly favors the Monte Carlo distributions for the $K_{1}^{-}$states over those of the non- $K_{1}^{-}$models. From the relative magnitude of the CKM matrix elements $\left|V_{u e}\right|$ and $\left|V_{u d}\right|$, we expect the branching fraction for the $\bar{u} s$ decays of the $\tau^{-}$to be about $5 \%$ of that of the analogous $\bar{u} d$ decays. The branching fractions for the $\bar{u} d$ and $\bar{u} s$ pseudoscalar, vector, and axial-vector states are given in Table 4.8. Our measurement of $1.17{ }_{-0.37}^{+0.41 \%}$ for the total branching fraction of $\tau^{-} \rightarrow \nu_{\tau} K_{1}^{-}$is in good agreement with the existing pattern of branching ratios to resonant states. 


\section{Chapter 5}

\section{Global Measurement of $\tau^{-}$ Branching Fractions}

Measurements of the branching fractions for the decays of the $\tau^{-}$lepton provide many insights concerning weak charged coupling and strong interactions below the $\tau^{-}$mass. Through the interaction of the $W^{-}$, the $\tau^{-}$decays to $\nu_{r}$ and an additional pair of leptons or quarks. Rates for the leptonic decays, $\tau^{-} \rightarrow e^{-} \bar{\nu}_{\mathrm{e}} \nu_{\tau}$ and $\tau^{-} \rightarrow \mu^{-} \vec{\nu}_{\mu} \nu_{r}$, are predicted very precisely by standard electroweak theory and their measurement allows a careful check of the universality of couplings among the three generations of leptons. Hadronic decays of the $\tau^{-}$are initiated with $W^{-}$ coupling to either $\bar{u} d$ or $\bar{u} s$ quark pairings. During the hadronization and strong decay process, additional $\tilde{u} u, \bar{d} d$, or $\bar{s} s$ quark pairs may be produced. Branching fraction measurements of these hadronic decays help reconstruct this process of strong interactions along two parallel paths characterized by the $\bar{u} d$ pairing with near-degenerate quark masses, and the $\bar{u} s$ pairing where the heavier $s$ quark breaks the mass symmetry.

This analysis gives a comprehensive set of measurements of the branching fractions for the decays of the $\tau^{-}$lepton. Although similar global measurements have been performed by the ALEPH [4] and CELLO [3] experiments, this is the first such analysis capable of separating charged $\pi$ and $K$. This allows a much more detailed set of decay categories and provides a means of separating the parallel physics of the $\bar{u} d$ and $\bar{u} s$ decays of the $\tau^{-}$. Another feature of this analysis is that only those events for which both decays are well-identified are included in the sample. While this strict requirement reduces the size of the sample, it allows the definition of event classes and the direct use of event selection efficiencies into the calculation of the branching fractions. Methods that use decay classes rather than event classes must assume that the mix of decays from the opposite half of the event is accurately estimated from a sum over branching fractions and event efficiencies. By requiring two well-identified decays and directly using the event efficiencies to determine the expected number of events in each event class, we 
obtain an over-determined system of equations that allows checks of the internal consistency of the estimated event efficiencies.

\subsection{The Method}

In order to obtain a complete set of $\tau^{-}$branching fractions, we first devise a set of selection criteria that will give a relatively pure sample of $\tau^{+} \tau^{-}$events and then classify each of those events according to the charged particles and photons observed in the $\tau^{+}$and $\tau^{-}$decays. The decay classifications are designed to be as detailed as possible, given the capabilities of the detector and the size of the data set. Table 5.1 shows the classification scheme, as well as the physical decay modes that are expected to contribute to each class. For most of the hadronic decay classes we have listed resonances through which those decays appear to proceed. Note that the $\bar{u} s$ decays through the $K^{*-}$ and $K_{1}^{-}$contribute to a number of classes, many of which are populated primarily by more common $\bar{u} d$ decay modes. Fortunately both the $K^{*-}$ and $K_{1}^{-}$have decays with $K^{-}$that serve as a distinct signature for the measurement of their branching fractions. We are then able to infer the $K^{*-}$ and $K_{1}^{-}$contributions to other predominantly $\bar{u} d$ classes. Although we refer to a $K_{1}^{-}$state, there are actually two states to consider, the $K_{1}^{-}(1270)$ and the $K_{1}^{-}(1400)$, as discussed in the previous chapter. Since the selection efficiencies and decay fractions for the $K^{-} \pi^{+} \pi^{-}, K^{-} \pi^{0} \pi^{0}$, and $K^{0} \pi^{0} \pi^{-}$final states are very similar for both of the $K_{1}^{-}$states, we are able to handle the $K_{1}^{-}$states together and report values for combined $K_{1}^{-}$.

The excellent particle identification of the TPC/Two-Gamma detector allows an efficient separation of all possible species of charged particles, namely $e, \mu, \pi$, and $K$. Although all of these species are found in one-prong decays, only $\pi$ and $K$ are possible in decays with higher charged multiplicity, since the leptonic decays produce a single charged particle. Preliminary feasibility studies with our data indicate that there are simply not any reasonable candidates for certain charged particle combinations that would allow a measurement of any corresponding branching fractions. These combinations, $\pi^{-} K^{+} \pi^{-}, K^{-} \pi^{+} K^{-}$, and $K^{-} K^{+} K^{-}$, are expected to be highly-suppressed and we do not include them in our analysis.

The neutrals classification is simply an estimate of the number of $\pi^{\circ} \mathrm{s}$ in the decay. This estimate comes from adding the number of reconstructed $\pi^{0} \mathbf{s}$ and the number of additional energetic photons associated with the decay. Again, preliminary feasibility checks of the $\tau^{+} \tau^{-}$sample give us an idea of which $\pi^{0}$ classifications will be possible for a given charged particle class. The number of decays with one charged pion is quite large and is separated into classes with 0,1 , 2 , or $\geq 3 \pi^{0} \mathrm{~s}$. Charged kaons are much less common and are separated into classes with 0,1 , or $\geq 2 \pi^{0} \mathrm{~s}$. Decays with $\pi^{-} \pi^{+} \pi^{-}$are separated into classes with 0 or $\geq 1 \pi^{0} \mathrm{~s}$. The three-prong decays with charged kaons, $K^{-} \pi^{+} \pi^{-}$and $K^{-} K^{+} \pi^{-}$, are 


\begin{tabular}{cllcll}
\hline \multirow{2}{*}{ Decay Class } & \multicolumn{2}{c}{ Decay Definition } & \multicolumn{2}{c}{ Decay Modes } \\
& & Charged & Neutral & Dominant & Additional \\
\hline 1 & $e^{-}$ & $e^{-}$ & $\geq 0$ & $e^{-}$ & \\
2 & $\mu^{-}$ & $\mu^{-}$ & $\geq 0$ & $\mu^{-}$ & \\
3 & $\pi^{-}$ & $\pi^{-}$ & 0 & $\pi^{-}$ & $K^{*-} \rightarrow \pi^{-} K_{L}^{0}$ \\
4 & $\pi^{-} \pi^{0}$ & $\pi^{-}$ & 1 & $\rho^{-}$ & $K_{1}^{-} \rightarrow \pi^{-} \pi^{0} K_{L}^{0}$ \\
5 & $\pi^{-} \pi^{0} \pi^{0}$ & $\pi^{-}$ & 2 & $a_{1}^{-}$ & $K^{*-} \rightarrow \pi^{-} K_{S}^{0}$ \\
6 & $\pi^{-} \pi^{0} \pi^{0} \pi^{0}$ & $\pi^{-}$ & $\geq 3$ & $\pi^{-} \pi^{0} \pi^{0} \pi^{0}$ & $K_{1}^{-} \rightarrow \pi^{-} \pi^{0} K_{S}^{0}$ \\
7 & $K^{-}$ & $K^{-}$ & 0 & $K^{-}$ & \\
8 & $K^{-} \pi^{0}$ & $K^{-}$ & 1 & $K^{*-}$ & \\
9 & $K^{-} \pi^{0} \pi^{0}$ & $K^{-}$ & $\geq 2$ & $K_{1}^{-}$ & \\
10 & $\pi^{-} \pi^{+} \pi^{-}$ & $\pi^{-} \pi^{+} \pi^{-}$ & 0 & $a_{1}^{-}$ & $K^{*-} \rightarrow \pi^{-} K_{S}^{0}$ \\
11 & $\pi^{-} \pi^{+} \pi^{-} \pi^{0}$ & $\pi^{-} \pi^{+} \pi^{-}$ & $\geq 1$ & $\pi^{-} \pi^{+} \pi^{-} \pi^{0}$ & $K_{1}^{-} \rightarrow \pi^{-} \pi^{0} K_{S}^{0}$ \\
12 & $K^{-} \pi^{+} \pi^{-}$ & $K^{-} \pi^{+} \pi^{-}$ & $\geq 0$ & $K_{1}^{-}$ & \\
13 & $K^{-} K^{+} \pi^{-}$ & $K^{-} K^{+} \pi^{-}$ & $\geq 0$ & $K^{-} K^{+} \pi^{-}$ & \\
\hline
\end{tabular}

Table 5.1: $\tau^{-}$decay classes and the decay channels that are expected to contribute to each class. The neutral classification is an estimate of the number of $\pi^{0} \mathrm{~s}$, based on the number of reconstructed $\pi^{0} \mathrm{~s}$ plus the number of additional energetic photons.

too rare to allow separate classes with differing numbers of $\pi^{0}$.

Now that we have our list of decay classes, we consider the physical decay modes that contribute to these classes. The modes that we use for modeling the decays of the $\tau^{-}$are listed in Table 5.2. Here we are still considering the ideal situation in which each of the decays is correctly classified based on the decay products. Later we will address the question of misclassification of decays. Note that more than one physical decay channel may contribute to a given decay class. For example, although we expect that the $\pi^{-}$class is mainly comprised of decays in which the $W^{-}$couples to $\bar{u} d$ quark pair in the pseudoscalar $\pi^{-}$state, we also know that there will be a small contribution from $W^{-}$coupling to $\bar{u} s$ quarks in the vector $K^{*-}$ state, followed by decay of the $K^{*-}$ to $\pi^{-}$, which we observe, and $K_{L}^{0}$, which we do not observe. If we assume that the $(K \pi)^{-}$decay proceeds through the $K^{*-}$ channel, then we are able to calculate the ratio of $K^{-} \pi^{0}$ and $\pi^{-} K^{0}$ decays from isospin symmetry. From the distinct signature of the $K^{-} \pi^{0}$ decays we can then estimate the $\pi^{-} K^{0}$ contribution to the $\pi^{-}$class. We follow an analogous argument for the $(K \pi \pi)^{-}$decays and the $K_{1}^{-}$resonances.

In order to relate the observed event class populations to the branching fractions, we require estimates of selection efficiencies for the possible decay modes. These efficiencies are calculated from Monte Carlo methods in which we generate 


\begin{tabular}{llll}
\hline & Decay Mode & Particles & Decay Class \\
\hline Leptons & $e^{-}$ & $e^{-} \bar{\nu}_{e}$ & $e^{-}$ \\
& $\mu^{-}$ & $\mu^{-} \bar{\nu}_{\mu}$ & $\mu^{-}$ \\
\hline \multirow{2}{*}{ Pseudoscalar } & $\pi^{-}(\bar{u} d)$ & $\pi^{-}$ & $\pi^{-}$ \\
\cline { 2 - 4 } & $K^{-}(\bar{u} s)$ & $K^{-}$ & $K^{-}$ \\
\hline \multirow{2}{*}{$\rho^{-}(\bar{u} d)$} & $\pi^{-} \pi^{0}$ & $\pi^{-} \pi^{0}$ \\
\cline { 2 - 4 } & $K^{*-}(\bar{u} s)$ & $K^{-} \pi^{0}$ & $K^{-} \pi^{0}$ \\
& & $\pi^{-} K_{L}^{0}$ & $\pi^{-}$ \\
\hline Axialvector & $a_{1}^{-}(\bar{u} d)$ & $\pi^{-} K_{S}^{0}$ & $\pi^{-} \pi^{0} \pi^{0}$ \\
& & $\pi^{-} K_{S}^{0}$ & $\pi^{-} \pi^{+} \pi^{-}$ \\
\cline { 2 - 4 } & $K_{1}^{-}(\bar{u} s)$ & $\pi^{-} \pi^{0} \pi^{0}$ & $\pi^{-} \pi^{0} \pi^{0}$ \\
& & $K^{-} \pi^{0} \pi^{0}$ & $\pi^{-} \pi^{+} \pi^{-}$ \\
\hline & & $K^{-} \pi^{+} \pi^{-}$ & $K^{-} \pi^{0}$ \\
& & $\pi^{-} \pi^{0} K_{L}^{0}$ & $\pi^{-} \pi^{0}$ \\
& & $\pi^{-} \pi^{0} K_{S}^{0}$ & $\pi^{-} \pi^{0} \pi^{0} \pi^{0}$ \\
& & $\pi^{-} \pi^{0} K_{S}^{0}$ & $\pi^{-} \pi^{+} \pi^{-} \pi^{0}$ \\
\hline Others & & $\pi^{-} \pi^{0} \pi^{0} \pi^{0}$ & $\pi^{-} \pi^{0} \pi^{0} \pi^{0}$ \\
& & $\pi^{-} \pi^{+} \pi^{-} \pi^{0}$ & $\pi^{-} \pi^{+} \pi^{-} \pi^{0}$ \\
& & $K^{-} K^{+} \pi^{-}$ & $K^{-} K^{+} \pi^{-}$ \\
\hline
\end{tabular}

Table 5.2: $\tau^{-}$decay modes considered in this analysis. The estimation of efficiencies is based upon simulations that are predominantly modeled through resonances for the hadronic decays, which are listed in $\bar{u} d$ and $\bar{u} s$ pairs.

random pairings of the various decay modes for $e^{+} e^{-} \rightarrow \tau^{+} \tau^{-}$events and then pass these events through a simulation of the detector. Events are generated with the $\tau^{+} \tau^{-}$generator KORALB [31] to which we have added decays through the $K_{1}^{-}$ resonances. The lepton classes, $e^{-}$and $\mu^{-}$, are assumed to be populated entirely by the two corresponding leptonic decays of the $\tau^{-}$, which make up about $35 \%$ of the $\tau^{-}$decay rate. The matrix elements for these decays are predicted by electroweak theory, so there is little uncertainty in the event simulation. The $\pi^{-}$and $K^{-}$are the well-established $\bar{u} d$ and $\bar{u} s$ pseudoscalar decays of the $\tau^{-}$and are modeled very precisely from the parameters of the decays $\pi^{-} \rightarrow \mu^{-} \bar{\nu}_{\mu}$ and $K^{-} \rightarrow \mu^{-} \bar{\nu}_{\mu}$. The $(\pi \pi)^{-}$and $(K \pi)^{-}$decays are modeled through the vector states $\rho^{-}$and $K^{*-}$, while the $(\pi \pi \pi)^{-}$and $(K \pi \pi)^{-}$decays are modeled through the axialvector $a_{1}^{-}$and $K_{1}^{-}$states. For the $\pi^{-} \pi^{0} \pi^{0} \pi^{0}, \pi^{-} \pi^{+} \pi^{-} \pi^{0}$, and $K^{-} K^{+} \pi^{-}$we use a simple matrix element, in which the hadronic current is proportional to the sum of the momenta of the mesons. 
The calculation of branching fractions in this analysis comes from an extended maximum likelihood fit to the observed event class populations. These event populations are assumed to follow Poisson statistics with the expected mean number of $\tau^{+} \tau^{-}$events in each event class given by,

$$
\nu_{i j}^{\tau \bar{\tau}}=\mathcal{L} \sigma_{\tau \neq} \sum_{k} \sum_{l} T_{i j \leftarrow k l}^{\tau \bar{\tau}} B_{k} B_{l} .
$$

The total number of $\tau^{+} \tau^{-}$events produced during the experimental runs, $N_{\tau^{+} \tau^{-}}$, is given by the product of the integrated luminosity $\mathcal{L}$, and the cross section for $\tau^{+} \tau^{-}$ production $\sigma_{\tau \bar{\tau}}$. The $B_{k}, B_{l}$ are the branching fractions for the decays of the $\tau^{+} \tau^{-}$ pair. The $T_{i j-k l}$ are the elements of the classification efficiency matrix. These are estimates of the fraction of $\tau^{+} \tau^{-}$events with decays $\tau^{+} \rightarrow k^{+} / \tau^{-} \rightarrow l^{-}$that will be observed in event class $i j$. The elements $T_{i j \leftarrow k l}$ are determined from Monte Carlo. The expected number of events in each class must also include background from physics other than $e^{+} e^{-} \rightarrow \tau^{+} \tau^{-}$. These are also arrived at through Monte Carlo. The equations,

$$
\begin{aligned}
\nu_{i j}^{e \bar{e}} & =\mathcal{L} \sigma_{e \bar{e}} T_{i j}^{e \bar{e}} \\
\nu_{i j}^{\mu \bar{\mu}} & =\mathcal{L} \sigma_{\mu \bar{\mu}} T_{i j}^{\mu \bar{\mu}} \\
\nu_{i j}^{q \bar{q}} & =\mathcal{L} \sigma_{q \bar{q}} T_{i j}^{q \bar{q}} \\
\nu_{i j}^{\gamma \gamma} & =\mathcal{L} \sigma_{\gamma \gamma} T_{i j}^{\gamma \gamma}
\end{aligned}
$$

give the expected number of background events from $e^{+} e^{-}, \mu^{+} \mu^{-}, q \bar{q}$, and $\gamma \gamma$ background events. For each event class we calculate an expected number of events by summing the various physical processes,

$$
\nu_{i j}=\nu_{i j}^{\tau \bar{\gamma}}+\nu_{i j}^{e \bar{e}}+\nu_{i j}^{\mu \bar{\mu}}+\nu_{i j}^{q \bar{q}}+\nu_{i j}^{\gamma \gamma}
$$

Assuming that the observed populations for the event classes, $n_{i j}$, are Poisson distributed, we construct an extended maximum likelihood expression,

$$
\mathcal{L}_{E}=\prod_{i j}\left(e^{-\nu_{i j}} \frac{\nu_{i j}^{n_{i j}}}{n_{i j} !}\right)
$$

Maximization of this likelihood function determines the most probable values for the branching fractions. Note that $\nu_{i j}$ scales with the integrated luminosity. This means that we may set the integrated luminosity to the measured value for the experiment and then check if the branching ratios for the decay modes add to unity within errors. An alternative approach is to normalize the branching fractions to unity in order to arrive at an independent measurement of the integrated luminosity based on the $\tau^{+} \tau^{-}$sample.

An outline of the analysis procedure is given in Figure 5.1 which shows how both the data and Monte Carlo events are passed through the selection and classification 
Data

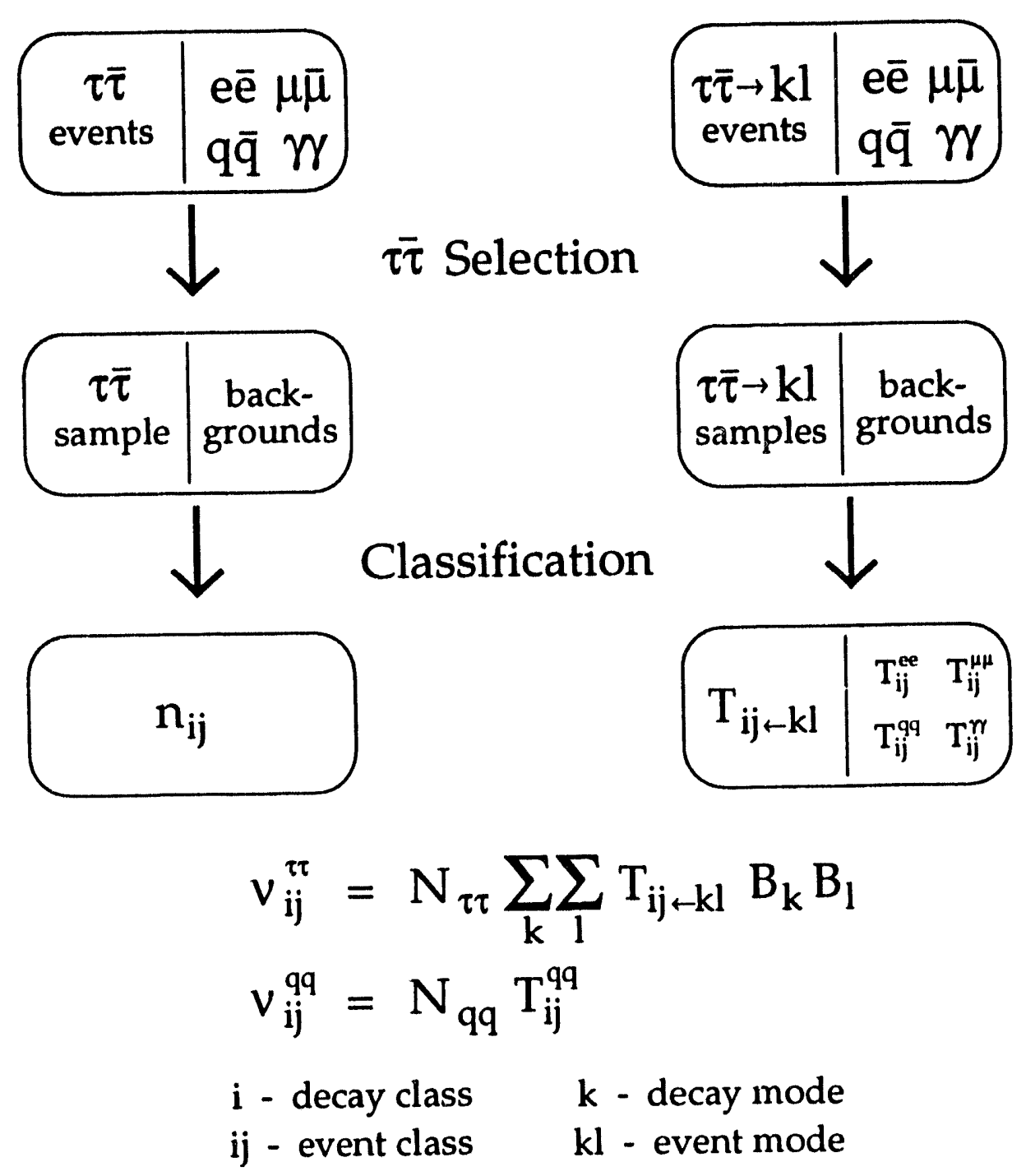

Monte Carlo

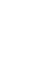


objective of the parallel Monte Carlo analysis is to obtain estirnates of the classification efficiencies for both $\tau^{+} \tau^{-}$and background events. With the Monte Carlo we are able to look at individual decay combinations for $\tau^{+} \tau^{-}$events and also at the individual sources of background to find out what fraction of each type of event is ending up in each event class. For the $\tau^{+} \tau^{-}$events these classification efficiencies are the elements $T_{i j \leftarrow k l}^{+7}$. For the background events we obtain a set of $T_{i j}$ for each of the expected background types. The details of the selection and classification of events, as well as the calculation of branching fractions, follow in the next several sections.

\subsection{Event Selection}

During the runs of the TPC/Two-Gamma experiment millions of events were recorded. Scattered among these events are thousands of $\tau^{+} \tau^{-}$events. Here we describe the selection criteria used to produce an event sample which retains as many of these $\tau^{+} \tau^{-}$events as possible while rejecting as many of the other types of events as possible. The data set used in this analysis in limited to the 1985-1986 high-field experimental run, which has an integrated luminosity of $63 \pm 7 \mathrm{pb}^{-1}$. The selection places more emphasis on purity than on efficiency. Consequently we will find that while our final sample consists of roughly $95 \% \tau^{+} \tau^{-}$events, it includes only about $15 \%$ of the estimated $8600 \tau^{+} \tau^{-}$events that occurred during the experimental run.

\subsubsection{Selection Criteria}

The selection criteria are based entirely on the kinematic properties and particle identification of the charged tracks of the events. No neutral information from the calorimeters is used in selecting the $\tau^{+} \tau^{-}$sample. Roughly, the idea is to select events with the characteristic topology of $\tau_{1+1}$ and $\tau_{1+3}$ events, where the subscripts give the numbers of charged tracks in each of the $\tau^{+} \tau^{-}$decays. The higher multiplicity $\tau^{+} \tau^{-}$events, such as $\tau_{1+5}$ and $\tau_{3+3}$, are much rarer and more difficult to separate from $q \bar{q}$ backgrounds.

The decay classification that we have described requires that the one-prong and three-prong tracks of these events must pass through regions of the detector where we are able to make an efficient separation into charged $e, \mu, \pi$, and $K$ candidates. Since the classification also depends on an estimate of the number of $\pi^{0}$ in each decay, we need to ensure that photons from the $\pi^{0}$ decays will have a reasonable probability of identification in the calorimeter. In the coverage of the detector components, there are gaps between the central and forward regions for both the electromagnetic calorimeters and muon chambers. In addition, at very low angles the TPC resolution worsens due to the decreased number of wire samples. For 
these reasons the selection for this analysis is limited to those events for which both groups of decay particles pass through the central region of the detector.

\subsubsection{Kinematic Selection}

The first step in the selection of $\tau_{1+1}$ and $\tau_{1+3}$ event samples is a series of kinematic and topological selections.

\section{$\tau_{1+1}$ Events}

The $\tau_{1+1}$ events are characterized by their low charged track multiplicity. At PEP energies, $q \bar{q}$ events have much higher charged multiplicities and are easily excluded from the $\tau_{1+1}$ sample. The potential backgrounds here are from the QED events $e^{+} e^{-} \rightarrow e^{+} e^{-}(\gamma)$ and $e^{+} e^{-} \rightarrow \mu^{+} \mu^{-}(\gamma)$ which usually have a pair of nearly collinear charged tracks close to beam energy. Although cuts against energy and collinearity of the tracks are quite efficient at removing most of these backgrounds, the radiative versions may have very similar topologies to those of the $\tau_{1+1}$ events. This requires the use of particle identification cuts in which we remove events where both of the one-prong tracks are tagged as electron candidates, or both are tagged as muon candidates.

We should also note that many of the one-prong decays of the $\tau^{-}$include $\pi^{0} \mathrm{~s}$. About $15 \%$ of their decay photons are expected to interact with the detector material before reaching the TPC and produce an $e^{+} e^{-}$pair. In order to retain these $\tau^{+} \tau^{-}$events in our sample we must allow for total charged multiplicities that exceed two, and then separate the one-prong tracks from the conversion pairs. The $\tau_{1+1}$ selection begins by describing track definitions that will be used to identify the one-prong tracks and the conversion electron candidates. The selection is actually run twice with slightly different track and topology definitions in order to reject backgrounds that would survive in the margins of either pass by itself. Each track may be assigned to any combination of these categories, they are not mutually exclusive.

- Good Tracks

- The track momentum is greater than $0.3 \mathrm{GeV} / c(0.1 \mathrm{GeV} / c)$.

- The track angle relative to the beamline must be at least $35^{\circ}\left(20^{\circ}\right)$.

- The track extrapolates to within $2 \mathrm{~cm}(5 \mathrm{~cm})$ of the interaction point in a plane perpendicular to the beam axis and to within $5 \mathrm{~cm}$ $(10 \mathrm{~cm})$ of the interaction point along the beam direction.

- Conversion Electron 
- The closest distance to the track of the other conversion electron in the pair is less than $10.0 \mathrm{~cm}(7.5 \mathrm{~cm})$

- The invariant mass of the conversion $e^{+} e^{-}$pair is less than $0.20 \mathrm{GeV} / \mathrm{c}^{2}$ $\left(0.15 \mathrm{GeV} / c^{2}\right)$.

- Likely Electron

- The $\mathrm{d} E / \mathrm{d} x$ fit to the electron hypothesis is $\boldsymbol{\chi}_{\mathbf{e}}^{\mathbf{2}}<\mathbf{9 . 0}$.

- The $\mathrm{d} E / \mathrm{d} x$ fit to the any of the other charged particle hypotheses is $x^{2}>\mathbf{9 . 0}$.

We also include two topological definitions, acollinearity and acoplanarity, and give their limits for the two selection passes. The acollinearity,

$$
\alpha_{\text {linear }}=\cos ^{-1}\left(-\frac{\vec{p}_{1} \cdot \vec{p}_{2}}{\left|\vec{p}_{1}\right|\left|\vec{p}_{2}\right|}\right)
$$

gives a measure of how close the tracks come to being back-to-back, while the acoplanarity,

$$
\alpha_{\text {planar }}=\cos ^{-1}\left[\left(\frac{\vec{z} \times \vec{p}_{1}}{\left|\vec{z} \times \vec{p}_{1}\right|}\right) \cdot\left(\frac{\vec{z} \times \vec{p}_{2}}{\left|\vec{z} \times \vec{p}_{2}\right|}\right)\right],
$$

where $\vec{z}$ is the beam direction, is a measure of the difference in azimuth of the tracks. The limits for these quantities for the two selection passes are given below.

- The acollinearity of the one-prong tracks must be between $3^{\circ}\left(2^{\circ}\right)$ and $55^{\circ}$ $\left(70^{\circ}\right)$.

- The acoplanarity of the one-prong tracks must be between $1^{\circ}\left(1^{\circ}\right)$ and $55^{\circ}$ $\left(55^{\circ}\right)$.

The lower limits are used to reject $e^{+} e^{-}$and $\mu^{+} \mu^{-}$events which tend to be collinear and coplanar, while the upper limits are set to retain the majority of $\tau^{+} \tau^{-}$events.

The events of the $\tau_{1+1}$ sample must satisfy the following kinematic and topological criteria for both sets of track and topology definitions given above.

- The total number of reconstructed tracks must not exceed 8, while the number of good tracks must be in the range of 2 to 6 , inclusive. These multiplicity cuts eliminate most of the QCD events.

- The scalar sum of momentum for all charged tracks must be between 7.25 GeV/c and $22.0 \mathrm{GeV} / c$. This requirement removes high-energy background such as $e^{+} e^{-}$and $\mu^{+} \mu^{-}$and low-energy background such as $\gamma \gamma$ events. 
- The event sphericity [33] must be less than 0.06, and the scalar sum of momentum for all good tracks in each hemisphere defined by the sphericity axis must be greater than $0.65 \mathrm{GeV} / \mathrm{c}$.

- There must be only 1 pairing of good tracks that is both acollinear and acoplanar, according to the cuts given above. This defines the one-prong candidates in the event.

- The two one-prong candidates must have a net charge of zero.

- All other tracks must be part of reconstructed $e^{+} e^{-}$pairs from photon conversions or identified as likely electrons.

\section{$T_{1+3}$ Events}

These events, with a higher charged track multiplicity, are more easily separable from $e^{+} e^{-}$and $\mu^{+} \mu^{-}$events. However, there is now some ambiguity with $q \tilde{q}$ events. Events in the $\tau_{1+3}$ sample must pass the following kinematic criteria.

- Good Tracks

- The track momentum must be greater than $0.3 \mathrm{GeV} / \mathrm{c}$.

- The track angle relative to the beamline must be at least $30^{\circ}$.

- The track must extrapolate within $5 \mathrm{~cm}$ in a plane perpendicular the beam axis and within $10 \mathrm{~cm}$ along the beam axis of the interaction point.

- Conversion Electron

- The closest distance to the track of the other conversion electron in the pair must be less than $10.0 \mathrm{~cm}$.

- The invariant mass of the conversion $e^{+} e^{-}$pair must be less than $0.20 \mathrm{GeV} / \mathrm{c}^{2}$.

With these definitions of good tracks and conversion electron candidates, the $\tau_{1+3}$ events must pass the following kinematic criteria.

- The total number of reconstructed tracks must not be more than 9 . This multiplicity cut eliminates most of the QCD events.

- The scalar sum of momentum for all charged tracks is between $4.5 \mathrm{GeV} / \mathrm{c}$ and $24.0 \mathrm{GeV} / \mathrm{c}$. This requirements removes high-energy background such as $e^{+} e^{-}$and $\mu^{+} \mu^{-}$and low-energy background such as $\gamma \gamma$ events. 
- There must be exactly 4 good tracks, apart from identified conversion $e^{+} e^{-}$ pairs. This defines the one-prong and three-prong candidates in the event.

- The 4 good tracks must have a net charge of zero.

- The one-prong candidate must be at least $140^{\circ}$ and less than $178^{\circ}$ from each of the three-prong candidates.

- The invariant mass of the three-prong tracks, assuming $\pi$ masses, must be less than $2.0 \mathrm{GeV} / \mathrm{c}^{2}$. This is another strict cut against $q \bar{q}$ backgrounds.

- The invariant mass of all the reconstructed tracks, assuming $\pi$ masses, must be at least $3.0 \mathrm{GeV} / \mathrm{c}^{2}$.

\subsubsection{Particle Identification Selection}

After this kinematic selection the $\tau_{1+1}$ sample still consists of nearly half radiative $e^{+} e^{-}$events, based on Monte carlo estimates. There are also a large number of radiative $\mu^{+} \mu^{-}$events that pass the kinematic cuts. The $\tau_{1+3}$ kinematic selection also allows considerable numbers of $e^{+} e^{-}$background events to pass. Particle identification cuts are required to further reduce the backgrounds to manageable levels.

In order to ensure that the decays pass through the reliable regions for particle identification, we first impose a geometric cut on the samples. This cut is actually determined by the need to identify the photons from $\pi^{0} \mathrm{~s}$ in the events. The photon candidates for the events of the $\tau^{+} \tau^{-}$sample are easily associated with the charged tracks, as shown in Figure 5.4. In order to identify a high percentage of the photons in these events, we define a charged decay axis for each decay. For one-prong decays this axis is simply the direction of the momentum vector of the one-prong track at the interaction point. For three-prong decays we sum the momenta of the three three-prong tracks there.

- The charged decay axis for each of the decays must have an angle of at least $50^{\circ}$ from the beam line.

This requirement gives a high probability of photons in the decays passing through the planes of the central calorimeter and gives a roughly uniform efficiency for photon identification and $\pi^{\mathbf{0}}$ reconstruction throughout the allowed angular region, making the analysis less sensitive to uncertainties in the modeling of decay angular distributions. This also keeps the decays in the region of efficient charged particle identification. Finally, the $e^{+} e^{-} \rightarrow e^{+} e^{-}$background is greatly reduced since its differential cross section increases rapidly in the forward regions.

The charged tracks of the remaining events are now passed through a preliminary separation into charged $e, \mu$, and $h$ candidates, based on information from 


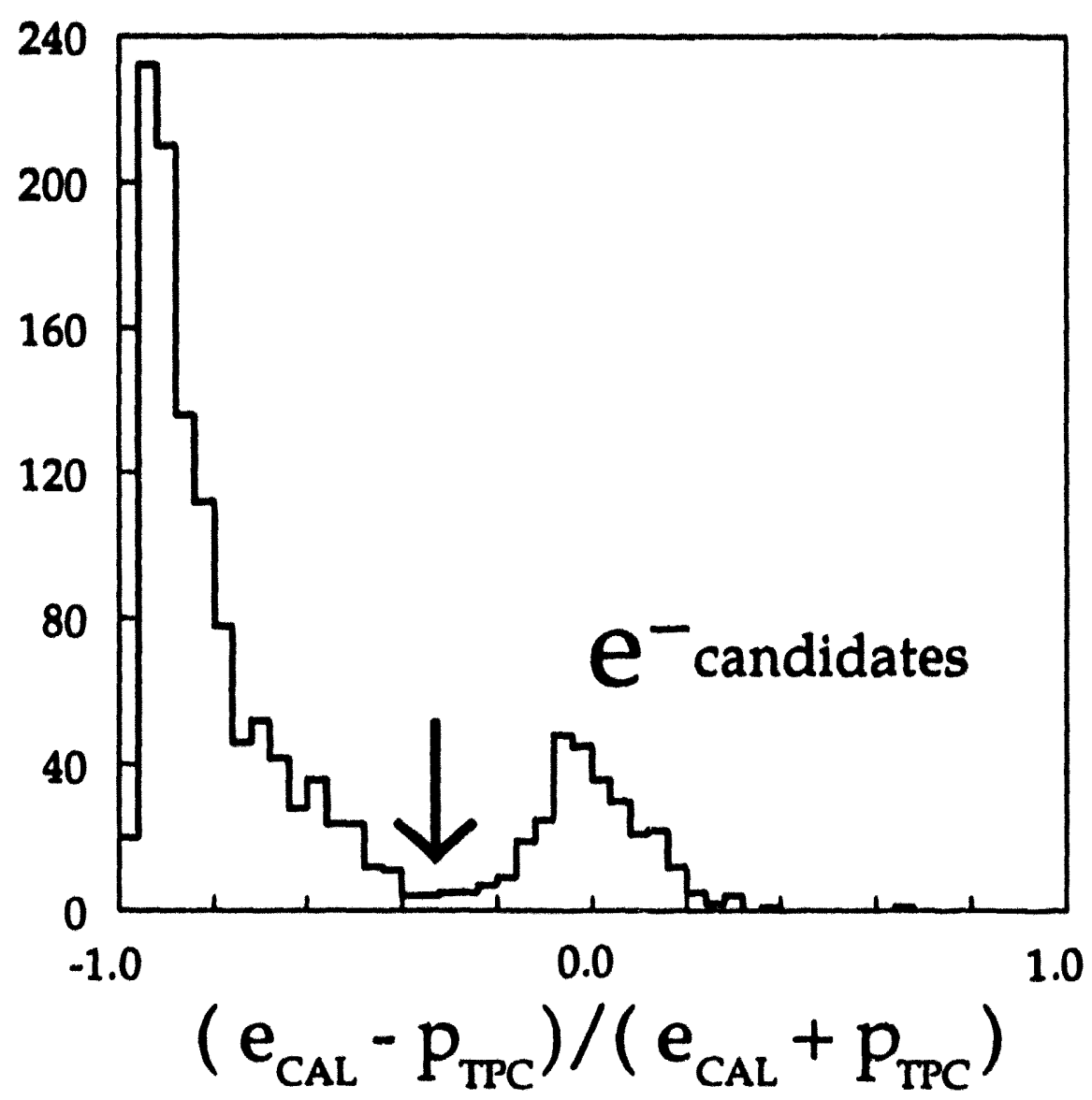

Figure 6.2: Energy/momentum function used to identify electron candidates in the preliminary $\tau^{+} \tau^{-}$sample. Note the peak centered about zero, the expected value for electrons.

the electromagnetic calorimeter and nuon chambers, followed by a more precise separation based on $\mathrm{d} E / \mathrm{d} x$ measurements from the time projection chamber. The hits in the muon chambers associated with the tracks are used to tag tracks as $\mu$ candidates. From its trajectory in the time projection chamber and our knowledge of the detector geometry and materials, each one-prong track is extrapolated through the layera of the muon chambers to predict the location of expected hits, given that the particle is a $\mu$. The actual pattern of hits is then compared with the expected hits from the extrapolation. In the central region, there are four layers of muon chambers, $a, b, c$, and $d$. The $\mu$ criteria are that the track have a hit in the terminal layer determined by the extrapolation, and there be no more than one missing hit in the previous layers. Tracks that satisfy this requirement are tagged as $\mu$ candidates.

Information from the electromagnetic calorimeter is used to tag tracks as $e$ candidates. Again, based on its trajectory in the time projection chamber, we 
extrapolate each one-prong and three-prong track to predict the position of intersection with the plane of the calorimeter. We then check for clusters near that point to indicate the actual position of the track in the calorimeter. Any cluster within $5.0 \mathrm{~cm}$ of the track is associated with that track and the estimated energy, assuming an electron, is assigned to that track. Figure 5.2 shows the sample of tracks plotted according to the function $\left(e_{C A L}-p_{T P C}\right) /\left(e_{C A L}+p_{T P C}\right)$. This expression places electrons near the zero point, since they shower, and other tracks toward the negative region, since they don't. The plot shows a prominent peak centered at zero with the remaining tracks appearing at lower regions. We define electron candidates as those tracks that fall above the arrow in the diagram, which corresponds to a calorimeter energy of at least $60 \%$ of the momentum measured in the TPC. As an example of the consistency of our integrated particle identification system, we note that none of the $\mu$ candidates defined above passes the $e$ criteria in the calorimeters. Unfortunately, there are many tracks for which we are unable to find an associated cluster in the calorimeter, either because of gaps in azimuth between modules or scattering that places the cluster outside the $5.0 \mathrm{~cm}$ limit for track association. Therefore, tracks that are not tagged as $\mu$ candidates must be considered to be $e$ candidates if there is no associated calorimeter cluster to determine shower energy.

The $e$ and $\mu$ tagging described above separates the track sample into three categories: electron, muon, or hadron. Plots of $\mathrm{d} E / \mathrm{d} x$ versus momentum from TPC measurements are given in Figure 5.3 for the preliminary $e, \mu$, and $h$ candidates. These $\mathrm{d} E / \mathrm{d} x$ values from the TPC allow us to further fine-tune each of these preliminary samples. The $\mathrm{d} E / \mathrm{d} x$ boundaries for the track regions are given in Table 5.3. Events with $e^{+} e^{-}$or $\mu^{+} \mu^{-}$tags are removed to give the final $\tau_{1+1}$ data sample. For the $\tau_{1+3}$ events, none of the three-prong tracks may be an $e$ candidate.

\subsubsection{Selection Efficiencies}

The total $\tau^{+} \tau^{-}$sample consists of the $\tau_{1+1}$ and $r_{1+3}$ samples. The efficiency of the $\tau^{+} \tau^{-}$selection and background estimates come from Monte Carlo simulations. Monte Carlo simulations of the various event types give values for the numbers of background events that still appear in the selected samples. The QED backgrounds $e^{+} e^{-} \rightarrow e^{+} e^{-}(\gamma)$ and $e^{+} e^{-} \rightarrow \mu^{+} \mu^{-}(\gamma)$ are well-understood physical events. On the other hand, $q \bar{q}$ and two-photon events are less well-understood and require some assumptions concerning the details of the decay process.

The efficiencies for the possible decay combinations of $\tau^{+} \tau^{-}$events are listed in Table 5.4 which gives efficiencies for the dominant decay modes for each of the decay classes, and in Table 5.5 which compares the efficiencies for the dominant and additional $\vec{u} s$ modes to give an idea of the possible systematic error introduced from differences in efficiency for different channels of the same decay class. Since 

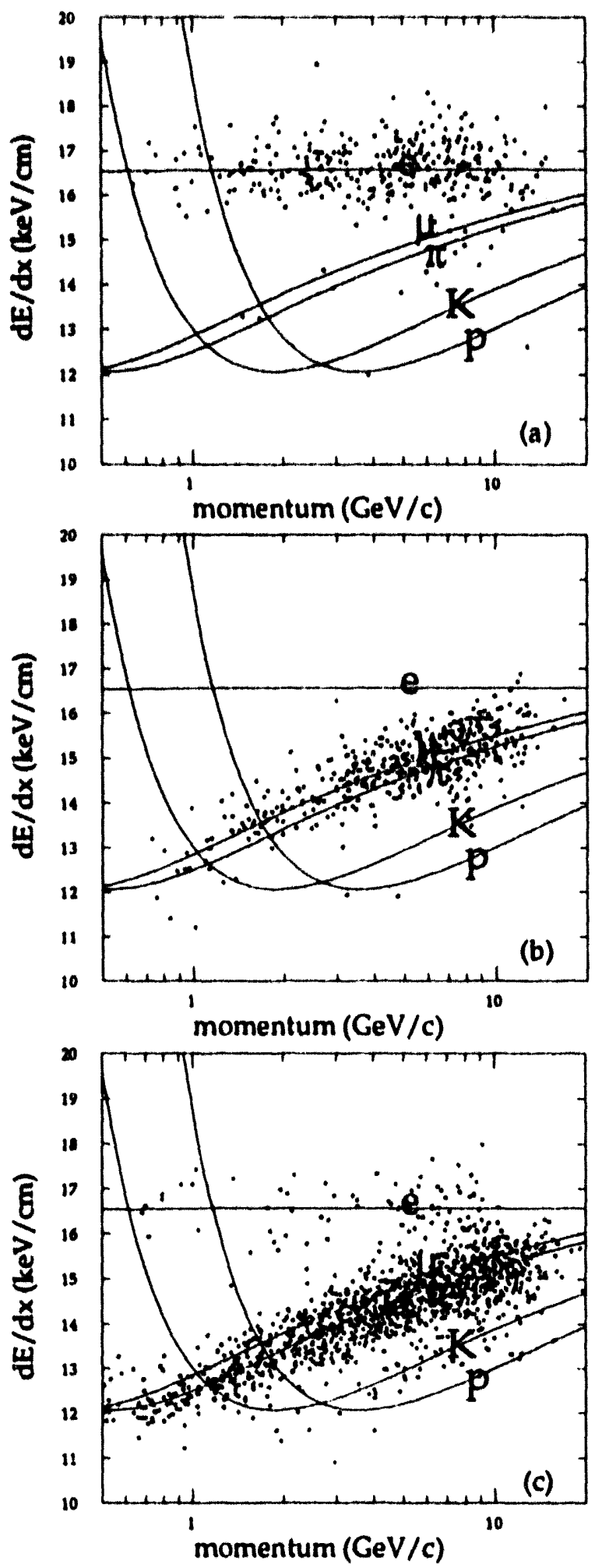

Figure 5.3: $\mathrm{d} E / \mathrm{d} x$ distributions for (a) $e^{-}$, (b) $\mu^{-}$, and (c) $h$ candidate tracks used in event selection. Electron and muon candidates are identified through information from the calorimeter and muon chambers. 


\begin{tabular}{lcll}
\hline & Preliminary Classification & \multicolumn{2}{c}{ Final Classification } \\
Class & CAL/MUC Criteria & Class & $\mathrm{d} E / \mathrm{d} x$ Criteria \\
\hline$e$ & Energy/momentum $>0.6$ or & $e$ & $\chi_{e}^{2}-\chi_{\pi}^{2}<10$ \\
& no CAL cluster or MUC hits & $h$ & $\chi_{e}^{2}-\chi_{\pi}^{2}>10$ \\
\hline$\mu$ & Hits through extrapolated & $\mu$ & $\chi_{\mu}^{2}-\chi_{k}^{2}<10$ \\
& layers of muon chambers & $h$ & $\chi_{\mu}^{2}-\chi_{K}^{2}>10$ \\
\hline$h$ & Not tagged as $e$ or $\mu$ & $e$ & $\chi_{e}^{2}-\chi_{\pi}^{2}<1$ \\
& & $h$ & $\chi_{e}^{2}-\chi_{\pi}^{2}>1$ \\
\hline
\end{tabular}

Table 6.3: Classification of one-prong and three-prong charged tracks into preliminary and final samples of $e, \mu$, and $h$ candidates based on calorimeter, muon chamber, and $\mathrm{d} E / \mathrm{d} x$ information. Events with $e^{+} e^{-}$or $\mu^{+} \mu^{-}$classification are excluded from the $\tau^{+} \tau^{-}$sample in order to remove QED backgrounds.

the efficiencies vary considerably from class to class, the makeup of the $\tau^{+} \tau^{-}$ sample is different from that of the original $\tau^{+} \tau^{-}$events.

\begin{tabular}{|c|c|c|c|c|c|c|c|c|c|c|c|c|c|c|}
\hline \multicolumn{15}{|c|}{ Event Selection Efficiency (\%) } \\
\hline \multicolumn{2}{|c|}{ Decay Class } & 1 & 2 & 3 & 4 & 5 & 6 & 7 & 8 & 9 & 10 & 11 & 12 & 13 \\
\hline$e^{-}$ & 1 & 0 & 18 & 14 & 11 & 11 & 11 & 19 & 16 & 13 & 22 & 24 & 27 & 24 \\
\hline$\mu^{-}$ & 2 & & 1 & 16 & 17 & 16 & 16 & 15 & 23 & 18 & 24 & 24 & 22 & 26 \\
\hline$\pi^{-}$ & 3 & & & 12 & 18 & 21 & 16 & 17 & 22 & 20 & 23 & 23 & 22 & 21 \\
\hline$\pi^{-} \pi^{0}$ & 4 & & & & 17 & 13 & 15 & 21 & 18 & 15 & 23 & 24 & 27 & 23 \\
\hline$\pi^{-} \pi^{0} \pi^{0}$ & 5 & & & & & 11 & 13 & 19 & 18 & 15 & 24 & 20 & 21 & 21 \\
\hline$\pi^{-} \pi^{0} \pi^{0} \pi^{0}$ & 6 & & & & & & 11 & 19 & 15 & 14 & 21 & 20 & 22 & 24 \\
\hline$K^{-}$ & 7 & & & & & & & 17 & 23 & 18 & 23 & 23 & 24 & 17 \\
\hline$K^{-} \pi^{0}$ & 8 & & & & & & & & 20 & 18 & 24 & 22 & 23 & 26 \\
\hline$K^{-} \pi^{0} \pi^{0}$ & 9 & & & & & & & & & 14 & 21 & 22 & 27 & 22 \\
\hline$\pi^{-} \pi^{+} \pi^{-}$ & 10 & & & & & & & & & & 0 & 1 & 1 & 1 \\
\hline$\pi^{-} \pi^{+} \pi^{-} \pi^{0}$ & 11 & & & & & & & & & & & 0 & 1 & 2 \\
\hline$K^{-} \pi^{+} \pi^{-}$ & 12 & & & & & & & & & & & & 0 & 1 \\
\hline$K^{-} K^{+} \pi^{-}$ & 13 & & & & & & & & & & & & & 0 \\
\hline
\end{tabular}

Table 5.4: Event selection efficiencies for the dominant channels of the event classes of $\tau^{+} r^{-}$decays, rounded to the nearest percent. The efficiencies for pairs of charge conjugate event classes are assumed to be identical. Note that there are small contributions from $\tau_{3+3}$ events that have been misidentified during the selection process. 


\begin{tabular}{|c|c|c|c|c|c|c|c|c|c|c|c|c|c|}
\hline \multicolumn{14}{|c|}{ Event Selection Efficiency \% } \\
\hline Decay Class & & $3 a$ & $3 b$ & $4 a$ & $4 b$ & $5 \mathrm{a}$ & $5 \mathrm{~b}$ & $6 a$ & $6 \mathrm{~b}$ & $10 a$ & $10 \mathrm{~b}$ & $11 \mathrm{a}$ & $11 \mathrm{~b}$ \\
\hline$e^{-}$ & 1 & 14 & 11 & 11 & 11 & 11 & 7 & 11 & 10 & 22 & 22 & 24 & 20 \\
\hline$\mu^{-}$ & 2 & 16 & 13 & 17 & 15 & 16 & 15 & 16 & 17 & 24 & 21 & 24 & 22 \\
\hline$\pi^{-}\left(\pi^{-}\right)$ & $3 a$ & 12 & 13 & 18 & 15 & 21 & 16 & 16 & 21 & 23 & 21 & 23 & 21 \\
\hline$\pi^{-}\left(K^{*-}\right)$ & $3 b$ & 13 & 6 & 11 & 11 & 11 & 7 & 13 & 11 & 23 & 18 & 25 & 22 \\
\hline$\pi^{-} \pi^{0}\left(\rho^{-}\right)$ & $4 a$ & 18 & 11 & 17 & 16 & 13 & 12 & 15 & 9 & 23 & 20 & 24 & 22 \\
\hline$\pi^{-} \pi^{0}\left(K_{1}^{-}\right)$ & $4 b$ & 15 & 11 & 16 & 9 & 12 & 10 & 9 & 11 & 23 & 22 & 23 & 20 \\
\hline$\pi^{-} \pi^{0} \pi^{0}\left(a_{1}^{-}\right)$ & $5 a$ & 21 & 11 & 13 & 12 & 11 & 11 & 13 & 13 & 24 & 18 & 20 & 21 \\
\hline$\pi^{-} \pi^{0} \pi^{0}\left(K^{*-}\right)$ & $5 b$ & 16 & 7 & 12 & 10 & 11 & 12 & 9 & 6 & 24 & 22 & 19 & 22 \\
\hline$\pi^{-} \pi^{0} \pi^{0} \pi^{0}$ & $6 a$ & 16 & 13 & 15 & 9 & 13 & 9 & 11 & 10 & 21 & 21 & 20 & 17 \\
\hline$\pi^{-} \pi^{0} \pi^{0} \pi^{0}\left(K_{1}^{-}\right)$ & $6 b$ & 21 & 11 & 9 & 11 & 13 & 6 & 10 & 8 & 18 & 17 & 19 & 18 \\
\hline$K^{-}$ & 7 & 17 & 16 & 21 & 18 & 19 & 24 & 19 & 20 & 23 & 21 & 23 & 21 \\
\hline$K^{-} \pi^{0}$ & 8 & 22 & 17 & 18 & 14 & 18 & 16 & 15 & 12 & 24 & 19 & 22 & 23 \\
\hline$K^{-} \pi^{0} \pi^{0}$ & 9 & 20 & 17 & 15 & 16 & 15 & 15 & 14 & 13 & 21 & 22 & 22 & 16 \\
\hline$\pi^{-} \pi^{+} \pi^{-}\left(a_{1}^{-}\right)$ & $10 a$ & 23 & 23 & 23 & 23 & 24 & 24 & 21 & 18 & 0 & 2 & 1 & 3 \\
\hline$\pi^{-} \pi^{+} \pi^{-}\left(K^{*-}\right)$ & $10 \mathrm{~b}$ & 21 & 18 & 20 & 22 & 18 & 22 & 21 & 17 & 2 & 4 & 2 & 2 \\
\hline$\pi^{-} \pi^{+} \pi^{-} \pi^{0}$ & $11 a$ & 23 & 25 & 24 & 23 & 20 & 19 & 20 & 19 & 1 & 2 & 0 & 1 \\
\hline$\pi^{-} \pi^{+} \pi^{-} \pi^{0}\left(K_{1}^{-}\right)$ & $11 \mathrm{~b}$ & 21 & 22 & 22 & 20 & 21 & 22 & 17 & 18 & 3 & 2 & 1 & 0 \\
\hline$K^{-} \pi^{+} \pi^{-}$ & 12 & 22 & 23 & 27 & 21 & 21 & 29 & 22 & 24 & 1 & 2 & 1 & 2 \\
\hline$K^{-} K^{+} \pi^{-}$ & 13 & 21 & 28 & 23 & 25 & 21 & 27 & 24 & 29 & 1 & 2 & 2 & 1 \\
\hline
\end{tabular}

Table 5.5: Event selection efficiencies for additional $\bar{u} s$ channels, compared with those of the $\bar{u} d$ channels that dominate those decay classes. For example, the $\bar{u} d$ contribution to $\pi^{-} \pi^{+} \pi^{-}$through the $a_{1}^{-}$channel is designated $10 \mathrm{a}$, while the $\bar{u} s$ contribution through the $K^{*-}$ channel is designated $10 \mathrm{~b}$. Resonant decay channels are given in parentheses.

\subsection{Event Classification}

After the selection of a relatively pure $\tau^{+} \tau^{-}$event sample, the next step is to classify each of the events in the sample according to the charged particles and photons associated with the $\tau^{+}$and $\tau^{-}$decay. The result of this process is a set of event class populations $n_{i j}$, where the $i$ and $j$ indices refer to the decay class for the $\tau^{+}$and $\tau^{-}$decays of the event. For example, if the $\tau^{+}$decay class is $e^{+}$ and the $\tau^{-}$decay class is $\mu^{-}$, the event class is $e^{+} \mu^{-}$. The populations for charge conjugate event classes, for example $e^{+} \mu^{-}$and $e^{-} \mu^{+}$, are added together to give a single class population.

In the following sections we describe the way in which the charged tracks are classified as $e^{ \pm}, \mu^{ \pm}, \pi^{ \pm}$, or $K^{ \pm}$, and the photon candidates from the calorimeter 
are used to obtain an estimate of the number of $\pi^{0} \mathrm{~s}$ in each decay.

\subsubsection{Charged Tracks}

The classification of the charged tracks in the $\tau^{+}$and $\tau^{-}$decays is based on momentum and $\mathrm{d} E / \mathrm{d} x$ values from the time projection chamber (TPC), energy measurements from the electromagnetic calorimeter (CAL), and the patterns of associated hits in the muon chambers (MUC). These quantities define a parameter space which we segregate into $e^{ \pm}, \mu^{ \pm}, \pi^{ \pm}$, and $K^{ \pm}$regions for one-prong tracks, and $\pi^{ \pm}$and $K^{ \pm}$regions for three-prong tracks. Our intention is to maximize the classification efficiency while keeping the boundary definitions as simple as possible. During the selection process certain regions of this particle identification space were excluded in order to remove background events. For example, $\tau_{1+1}$ events in which the one-prong tracks both fall within regions of high electron probability or both fall within regions of high muon probability are excluded from the sample in order to reject $e^{+} e^{-} \rightarrow e^{+} e^{-}(\gamma)$ and $e^{+} e^{-} \rightarrow \mu^{+} \mu^{-}(\gamma)$ background events.

The procedure for track classification is similar to that for the particle identification selection. In fact we use the preliminary classification where we have tagged one-prong charged tracks as $e, \mu$, or $h$. Again, the electron preliminary class consists of those tracks for which the energy measured in the calorimeter is at least $60 \%$ of the momentum measured in the TPC. Tracks without associated clusters in the calorimeter and without hits in the muon chambers must be considered electron candidates. The muon preliminary class consists of tracks that have hit patterns expected for a $\mu$ of that vector momentum. With the well-understood Gaussian distribution and excellent $\mathrm{d} E / \mathrm{d} x$ resolution of the TPC we are able to fine-tune these preliminary track classes to obtain final classifications. The $\mathrm{d} E / \mathrm{d} x$ boundaries for the track regions are given in Table 5.6. While in the selection the boundaries were defined loosely to ensure the removal of $e^{+} e^{-}$and $\mu^{+} \mu^{-}$events, here we choose boundaries that maximize the efficiency of our classification.

\subsubsection{Neutrals}

The neutral class for each decay is simply an estimate of the number of $\pi^{0} \mathrm{~s}$, based on the photons observed in the decay. Photons themselves must first be reconstructed from showers in the calorimeter or from conversion $e^{+} e^{-}$pairs in the TPC. In the calorimeter, photons generate electromagnetic showers which fire clusters of adjacent cells. The cluster from a given photon may have considerable overlap with clusters from other photons or from charged particles, especially for events such as $\tau^{-}$decays where the decay products are collimated due to the boost of the $\tau^{-}$at the energies of the PEP collider. Clusters that are not associated with a charged track are photon candidates. 


\begin{tabular}{lclc}
\hline & \multicolumn{2}{c}{ Charged Track Classification } \\
\hline \multirow{3}{*}{ Class } & Preliminary & Final \\
\hline$e$ & CAL/MUC Criteria & Ciass & $\mathrm{d} E / \mathrm{d} x$ Criteria \\
\hline & Energy/momentum $>0.6$ or & $e$ & $\chi_{e}^{2}-\chi_{\pi}^{2}<8$ \\
& no CAL cluster or MUC hits & $\pi$ & $\chi_{e}^{2}-\chi_{\pi}^{2}>8, \chi_{\pi}^{2}-\chi_{K}^{2}<4$ \\
& & $K$ & $\chi_{\pi}^{2}-\chi_{K}^{2}>4$ \\
\hline$\mu$ & Hits through extrapolated & $e$ & $\chi_{\mu}^{2}-\chi_{e}^{2}>10$ \\
& layers of muon chambers & $\mu$ & $\chi_{\mu}^{2}-\chi_{e}^{2}<10, \chi_{\mu}^{2}-\chi_{K}^{2}<9$ \\
& & $K$ & $\chi_{\mu}^{2}-\chi_{K}^{2}>9$ \\
\hline$h$ & Not tagged as $e$ or $\mu$ & $e$ & $\chi_{\pi}^{2}-\chi_{e}^{2}>6$ \\
& & $\pi$ & $\chi_{\pi}^{2}-\chi_{e}^{2}<6, \chi_{\pi}^{2}-\chi_{K}^{2}<4$ \\
& & $K$ & $\chi_{\pi}^{2}-\chi_{K}^{2}>4$ \\
\hline
\end{tabular}

Table 5.6: Classification of one-prong charged tracks into preliminary and final samples of $e, \mu$, and $h$ candidates based on calorimeter, muon chamber, and $\mathrm{d} E / \mathrm{d} x$ information.

Each photon candidate must be assigned to either the $\tau^{+}$or $\tau^{-}$decay in the event. Figure 5.4 shows the angular distribution of photons relative to the charged tracks for the events of the $\tau^{+} \tau^{-}$sample. The photons are easily associated with a particular decay with few ambiguous cases. A photon is assigned to a decay if the direction of the photon is within $45^{\circ}$ of the charged axis of the decay. For one-prong decays this axis is given by the momentum of the one-prong track at the interaction point, while for the three-prong case the axis is the vector sum of the three-prong tracks. We also require that the energy of the photon be greater than $0.4 \mathrm{GeV}$ to avoid the large number of clusters from split-offs and noise.

Once the group of photons has been established for the decay, we reconstruct as many $\pi^{0}$ candidates from the pool of photons as possible. Figure 5.5 shows the invariant mass combinations for all of the photon pairings. There is a distinct peak at the mass of the $\pi^{0}$. Photon pairings with masses between $0.07 \mathrm{GeV} / c^{2}$ and $0.20 \mathrm{GeV} / c^{2}$ are considered to be $\pi^{0}$ candidates. In the case that the same photon is included in two or more $\pi^{0}$ candidates, we use the particular combinations in which each photon appears no more than once and the total number of $\pi^{0}$ candidates is maximized.

Although many of these energetic photons are paired into $\pi^{0} \mathrm{~s}$, there are also many photons which are not. Pairs of photons may merge to give one cluster rather than two distinct clusters in the calorimeter, or one of the photons may be low energy and fail the selection criteria. Following this reasoning, each additional energetic photon is considered to be a signature for an additional $\pi^{0}$ in the decay. 


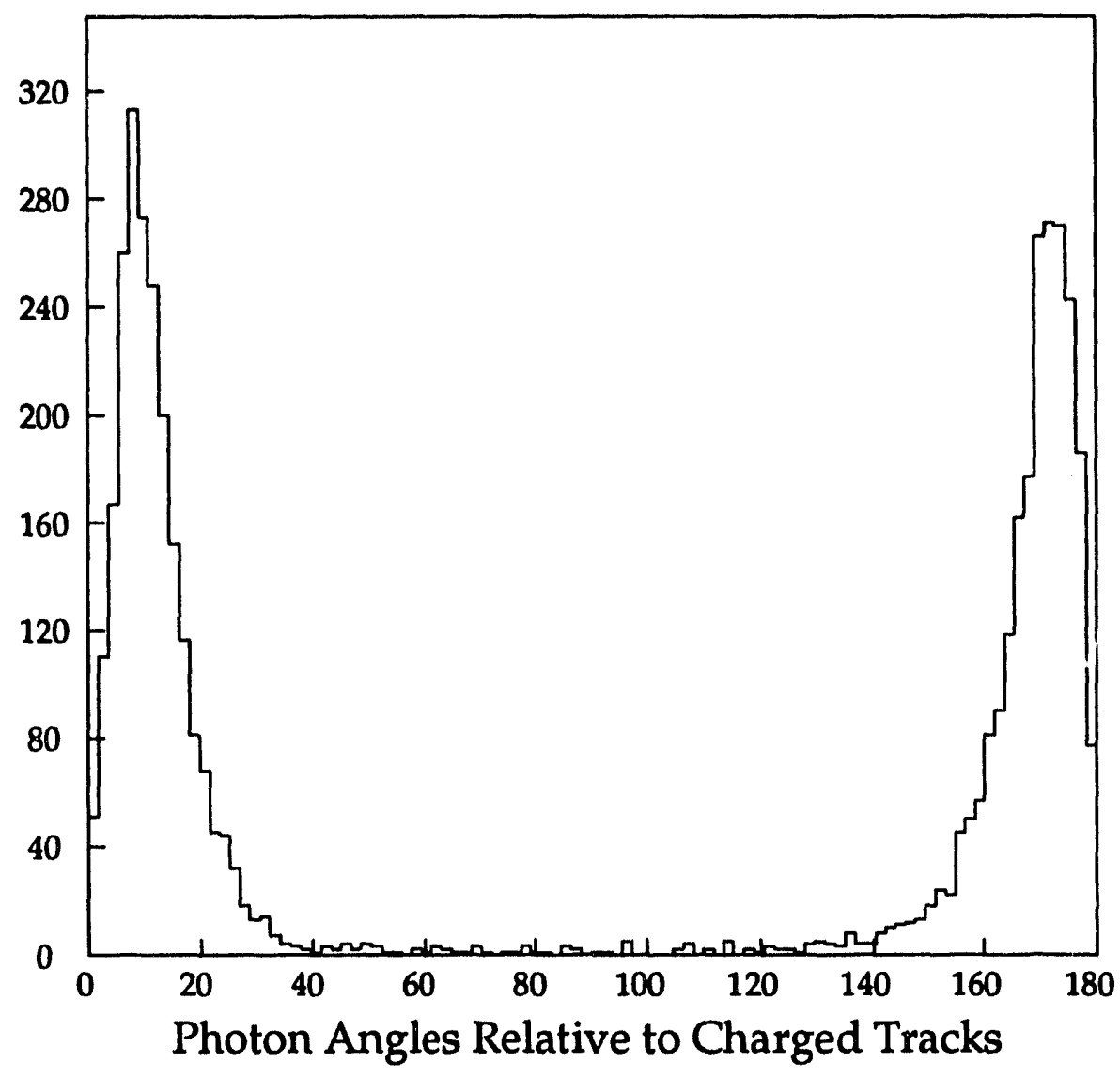

Figure 5.4: Angular distribution of photons relative to the charged particle axis for the decays of the $\tau^{+} \tau^{-}$sample.

This method of estimating the number of $\pi^{0}$ s gives a reasonable efficiency for correctly assigning the decay to its proper neutrals class. The results of Monte Carlo simulations of $\tau^{-} \rightarrow \nu_{\tau} \pi^{-}, \tau^{-} \rightarrow \nu_{\tau} \pi^{-} \pi^{0}, \tau^{-} \nu_{\tau} \rightarrow \pi^{-} \pi^{0} \pi^{0}$, and $\tau^{-} \rightarrow \nu_{\tau} \pi^{-} \pi^{0} \pi^{0} \pi^{0}$ are given in Table 5.7 to show the number of photons that we expect to be reconstructed in these decays. Using the additional energetic photons as signals for additional $\pi^{0}$ significantly increases our classification efficiency.

\subsubsection{Event Class Populations}

With each of the decays in the $\tau^{+} \tau^{-}$sample assigned to a charged and neutrals class, we consider how to combine this information to give a decay class. Table 5.8 gives the numbers of decays that fall into the various combinations. We note that although most of the decays in the electron class have no neutrals, there are quite a number with one or two. This is expected from the radiation of the low mass electrons as they pass through the material of the detector. All of these categories are considered to be in the $e^{-}$class. While almost all decays in the muon class 


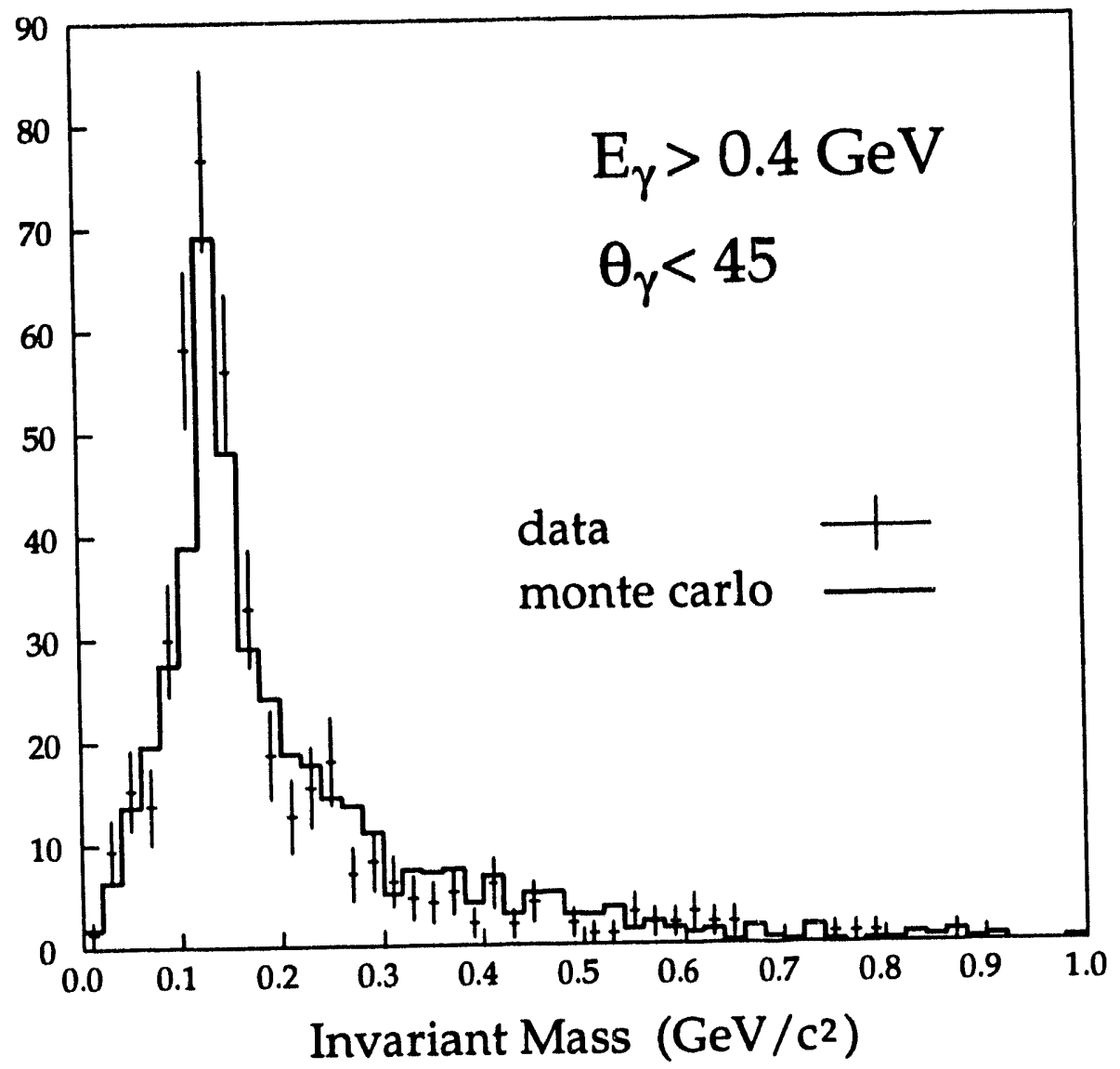

Figure 5.5: Invariant mass of photons pairs from the $\tau^{+} \tau^{-}$sample. There is a distinct peak centered at the mass of the $\pi^{0}$.

show no neutrals, there are some with multiple $\pi^{0}$. A closer look reveals that these muon decays with neutrals are at low momentum where the pion misidentification in the muon chambers is high. Monte Carlo simulations suggest that these decays are likely due to $\pi^{-}+\geq 1 \pi^{0}$ decays rather than $\mu^{-}$decays. Consequently, only those decays with one candidate $\mu$ and no neutrals are included in the $\mu^{-}$decay class. The decays with additional neutrals are combined with the $\pi^{-} \pi^{0}, \pi^{-} \pi^{0} \pi^{0}$, and $\pi^{-} \pi^{0} \pi^{0} \pi^{0}$ decay classes. The definitions of the remaining classes are straightforward.

According to the classification scheme we have described, we find the populations of the event classes as given in Table 5.9. These are the numbers $n_{i j}$ that will be used in our global fit of the branching fractions of the $\tau^{-}$lepton. For example, there are 63 events where one of the $\tau$ decays is classified as $\pi^{-} \pi^{0}$ (decay class 4 ) and the other as $\pi^{-} \pi^{+} \pi^{-}$(decay class 10$)$. The populations for $e^{+} / e^{-}$and $\mu^{+} / \mu^{-}$ events are zero since those event class regions are excluded in the selection process in order to remove $e^{+} e^{-} \rightarrow e^{+} e^{-}(\gamma)$ and $\mu^{+} \mu^{-} \rightarrow \mu^{+} \mu^{-}(\gamma)$ background events. 


\begin{tabular}{|c|c|c|c|c|c|c|}
\hline \multirow[t]{2}{*}{ Decay } & \multirow{2}{*}{$\begin{array}{c}\text { Number of } \\
\text { reconstructed } \pi^{\circ}\end{array}$} & \multicolumn{4}{|c|}{ Additional $\gamma$} & \multirow[t]{2}{*}{ Efficiency } \\
\hline & & 0 & 1 & 23 & & \\
\hline \multirow{4}{*}{$\pi^{-}$} & 0 & 97 & 3 & 0 & 5 & \multirow{4}{*}{$97 \%$} \\
\hline & 1 & 0 & 0 & 0 & 0 & \\
\hline & 2 & 0 & 0 & & & \\
\hline & 3 & 0 & 0 & & & \\
\hline \multirow{4}{*}{$\pi^{-} \pi^{0}$} & 0 & 17 & 44 & 6 & 0 & \multirow{4}{*}{$73 \%$} \\
\hline & 1 & 29 & 3 & 0 & 0 & \\
\hline & 2 & 0 & 0 & 0 & 0 & \\
\hline & 3 & 0 & 0 & 0 & 0 & \\
\hline \multirow{4}{*}{$\pi^{-} \pi^{0} \pi^{0}$} & 0 & 5 & 19 & $\overline{15}$ & 0 & \multirow{4}{*}{$51 \%$} \\
\hline & 1 & 11 & 26 & 5 & 0 & \\
\hline & 2 & 10 & 4 & 0 & 0 & \\
\hline & 3 & 1 & 0 & 0 & 0 & \\
\hline \multirow{4}{*}{$\pi^{-} \pi^{0} \pi^{0} \pi^{0}$} & 0 & 7 & 9 & 10 & 4 & \multirow{4}{*}{$33 \%$} \\
\hline & 1 & 10 & 12 & 10 & 2 & \\
\hline & 2 & 12 & 15 & 2 & 0 & \\
\hline & 3 & 4 & 1 & 1 & 0 & \\
\hline
\end{tabular}

Table 5.7: Distributions of reconstructed $\pi^{0}$ and additional photons for the $\pi^{-}, \pi^{-} \pi^{0}$, $\pi^{-} \pi^{0} \pi^{0}, \pi^{-} \pi^{0} \pi^{0} \pi^{0}$ decays, based on Monte Carlo simulation. The numbers are in percent and the bold values are for those fractions which are correctly assigned.

There are also no events with $\tau_{3+3}$ topologies since our selection is limited to events identified as $\tau_{1+1}$ or $\tau_{1+3}$ candidates.

\subsubsection{Classification Efficiencies}

With the event classification definitions and event populations in hand, we have a rough estimate of the relative frequency of the decays of the $\tau^{-}$. However, as noted before, the selection efficiency varies from mode to mode. An extreme example is for the $e^{+} e^{-}$and $\mu^{+} \mu^{-}$cases where the need to exclude $e^{+} e^{-} \rightarrow e^{+} e^{-}(\gamma)$ and $e^{+} e^{-} \rightarrow \mu^{+} \mu^{-}(\gamma)$ backgrounds requires us to use selection criteria that remove almost all of those types of $\tau^{+} \tau^{-}$events.

To obtain branching fraction estimates we fit a set of expected event populations to the actual event populations of the data. The expected populations are functions of the branching fractions and the selection efficiencies for each of the possible event types. Therefore we require an estimate of the fraction of events that will fall into each possible classification. The elements of the efficiency matrix, $T_{i j \leftarrow k l}^{\tau \neq}$, 


\begin{tabular}{lrrrr}
\hline & \multicolumn{4}{c}{ Neutrals } \\
Charged Tracks & 0 & \multicolumn{1}{c}{1} & 2 & 3 \\
\hline$e^{-}$ & 276 & 58 & 9 & 0 \\
$\mu^{-}$ & 386 & 21 & 5 & 2 \\
$\pi^{-}$ & 375 & 550 & 197 & 31 \\
$K^{-}$ & 22 & 21 & 7 & 2 \\
$\pi^{-} \pi^{+} \pi^{-}$ & 242 & 108 & 40 & 8 \\
$K^{-} \pi^{+} \pi^{-}$ & 13 & 7 & 0 & 0 \\
$K^{-} K^{+} \pi^{-}$ & 8 & 4 & 2 & 0 \\
\hline
\end{tabular}

Table 5.8: Decay class populations, based on charged and neutral particle classes.

\begin{tabular}{|c|c|c|c|c|c|c|c|c|c|c|c|c|c|c|}
\hline \multicolumn{15}{|c|}{ Event Class Populations } \\
\hline \multicolumn{2}{|c|}{ Decay Class } & 1 & 2 & 3 & 4 & 5 & 6 & 7 & 8 & 9 & 10 & 11 & 12 & 13 \\
\hline$e^{-}$ & 1 & 0 & 83 & 46 & 73 & 22 & 4 & 4 & 4 & 0 & 55 & 43 & 9 & 0 \\
\hline$\mu^{-}$ & 2 & & 0 & 68 & 113 & 26 & 7 & 1 & 4 & 3 & 50 & 27 & 3 & 1 \\
\hline$\pi^{-}$ & 3 & & & 33 & 87 & 34 & 6 & 3 & 1 & 1 & 40 & 21 & 2 & 0 \\
\hline$\pi^{-} \pi^{0}$ & 4 & & & & 64 & 48 & 4 & 4 & 5 & 1 & 63 & 42 & 3 & 0 \\
\hline$\pi^{-} \pi^{0} \pi^{0}$ & 5 & & & & & 7 & 3 & 3 & 1 & 0 & 31 & 15 & 3 & 2 \\
\hline$\pi^{-} \pi^{0} \pi^{0} \pi^{0}$ & 6 & & & & & & 0 & 0 & 0 & 0 & 3 & 6 & 0 & 0 \\
\hline$K^{-}$ & 7 & & & & & & & 0 & 1 & 1 & 0 & 5 & 0 & 0 \\
\hline$K^{-} \pi^{0}$ & 8 & & & & & & & & 0 & 0 & 3 & 2 & 0 & 0 \\
\hline$K^{-} \pi^{0} \pi^{0}$ & 9 & & & & & & & & & 0 & 3 & 0 & 0 & 0 \\
\hline$\pi^{-} \pi^{+} \pi^{-}$ & 10 & & & & & & & & & & 0 & 0 & 0 & 0 \\
\hline$\pi^{-} \pi^{+} \pi^{-} \pi^{0}$ & 11 & & & & & & & & & & & 0 & 0 & 0 \\
\hline$K^{-} \pi^{+} \pi^{-}$ & 12 & & & & & & & & & & & & 0 & 0 \\
\hline$K^{-} K^{+} \pi^{-}$ & 13 & & & & & & & & & & & & & 0 \\
\hline
\end{tabular}

Table 5.9: Event class populations for the $\tau^{+} \tau^{-}$sample. These are the $n_{i j}$ used in the fit that determines the branching fractions for the $\tau^{-}$decay classes. Note that all the potential $e^{+} e^{-}$or $\mu^{+} \mu^{-}$event candidates are removed in order to limit background from $e^{+} e^{-} \rightarrow e^{+} e^{-}(\gamma)$ and $e^{+} e^{-} \rightarrow \mu^{+} \mu^{-}(\gamma)$ events.

come from Monte Carlo models of $e^{+} e^{-} \rightarrow \tau^{+} \tau^{-}$events. For $i_{\text {max }}$ decay classes and $k_{\max }$ decay modes there will be $i_{\max }^{2} k_{\max }^{2}$ elements of this event efficiency matrix. For the thirteen decay classes and nineteen decay modes considered in this analysis, the number of elements is prohibitively large to list here. However, 


\begin{tabular}{|c|c|c|c|c|c|c|c|c|c|c|c|c|c|c|}
\hline \multicolumn{15}{|c|}{ Classification Efficiency (\%) } \\
\hline Decay $\mathrm{Cl} \varepsilon$ & & 1 & 2 & 3 & 4 & 5 & 6 & 7 & 8 & 9 & 10 & 11 & 12 & 13 \\
\hline$e^{-}$ & 1 & 0 & 0 & 2 & 8 & 1 & 0 & 0 & 0 & 0 & 0 & 0 & 0 & 0 \\
\hline$\mu^{-}$ & 2 & & 0 & 0 & 0 & 0 & 0 & 0 & 0 & 0 & 0 & 0 & 0 & 0 \\
\hline$\pi^{-}$ & 3 & & & 0 & 0 & 0 & 0 & 0 & 0 & 0 & 0 & 0 & 0 & 0 \\
\hline$\pi^{-} \pi^{0}$ & 4 & & & & 0 & 0 & 0 & 0 & 0 & 0 & 0 & 0 & 0 & 0 \\
\hline$\pi^{-} \pi^{0} \pi^{0}$ & 5 & & & & & 0 & 0 & 0 & 0 & 0 & 0 & 0 & 0 & 0 \\
\hline$\pi^{-} \pi^{0} \pi^{0} \pi^{0}$ & 6 & & & & & & 0 & 0 & 0 & 0 & 0 & 0 & 0 & 0 \\
\hline$K^{-}$ & 7 & & & & & & & 0 & 0 & 0 & 0 & 0 & 0 & 0 \\
\hline$K^{-} \pi^{0}$ & 8 & & & & & & & & 0 & 0 & 0 & 0 & 0 & 0 \\
\hline$K^{-} \pi^{0} \pi^{0}$ & 9 & & & & & & & & & 0 & 0 & 0 & 0 & 0 \\
\hline$\pi^{-} \pi^{+} \pi^{-}$ & 10 & & & & & & & & & & 0 & 0 & 0 & 0 \\
\hline$\pi^{-} \pi^{+} \pi^{-} \pi^{0}$ & 11 & & & & & & & & & & & 0 & 0 & 0 \\
\hline$K^{-} \pi^{+} \pi^{-}$ & 12 & & & & & & & & & & & & 0 & 0 \\
\hline$K^{-} K^{+} \pi^{-}$ & 13 & & & & & & & & & & & & & 0 \\
\hline
\end{tabular}

Table 5.10: Classification efficiencies of $\tau^{+} \rightarrow \bar{\nu}_{\tau} \pi^{+} \pi^{0} / \tau^{-} \rightarrow \nu_{\tau} \bar{\nu}_{e} e^{-}$decays, estimated from Monte Carlo simulation. Note that there is no significant misidentification of the charged $e$ or $\pi$, while there is considerable misidentification of the number of $\pi^{0} \mathrm{~s}$ in the event.

as an example, Table 5.10 gives the values for the elements $T_{i j-\left(\pi^{+} \pi^{0}\right)\left(e^{-}\right)}^{\tau \pi}$ for the process $\tau^{+} \rightarrow \bar{\nu}_{\tau} \pi^{+} \pi^{0} / \tau^{-} \rightarrow \nu_{\tau} \bar{\nu}_{e} e^{-}$, one of the most probable event types. Note that only a few decay classes contain any significant fraction of these events. Because of the excellent charged particle identification possible with the combination of calorimeter and TPC, only about $1 \%$ of the $e$ will be misidentified. On the other hand, the less certain estimation of the number of $\pi^{0} \mathrm{~s}$ is evident from the fraction of $\pi^{-} \pi^{0}$ events that are identified as having 0 or $2 \pi^{0} \mathrm{~s}$. This pattern is typical of the charged and neutral classification of all the decay modes.

\subsection{Branching Fractions}

All of the pieces are now in place to allow the calculation of the branching fractions. Using the classification efficiency matrices estimated from Monte Carlo, we determine the expected number of $\tau^{+} \tau^{-}$events for each of the event classes according to

$$
\nu_{i j}^{\tau \pi}=\mathcal{L} \sigma_{\tau \mp} \sum_{k} \sum_{l} T_{i j-k l}^{\tau \pi} B_{k} B_{l}
$$


while the expected number of background events in each class is given b/,

$$
\begin{aligned}
\nu_{i j}^{e d} & =\mathcal{L} \sigma_{e \bar{e}} T_{i j}^{e d} \\
\nu_{i j}^{\mu \bar{d}} & =\mathcal{L} \sigma_{\mu \bar{p}} T_{i j}^{\mu \bar{A}} \\
\nu_{i j}^{q \phi} & =\mathcal{L} \sigma_{q d} T_{i j}^{q d} \\
\nu_{i j}^{\gamma \gamma} & =\mathcal{L} \sigma_{\gamma \gamma} T_{i j}^{\gamma \gamma} .
\end{aligned}
$$

The total expected number of events for each of the event classes is then

$$
\nu_{i j}=\nu_{i j}^{T \mp}+\nu_{i j}^{e d}+\nu_{i j}^{\mu \AA}+\nu_{i j}^{q q}+\nu_{i j}^{T \gamma} \text {. }
$$

Given the expected number of events for each class, the likelihood of observing a particular set of event class populations, $n_{i j}$, is given by the expression

$$
\mathcal{L}_{E}=\prod_{i j}\left(e^{-\nu_{1},} \frac{\nu_{i j}^{n_{1 j}}}{n_{i j} !}\right)
$$

This likelihood expression is a function of the $B_{k}$, the branching fractions of a comprehensive set of physical decay modes of the $\tau^{-}$lepton. The decay modes $k$ may be categorized in a number of ways. Each involves some assumption about the nature of the decays that contribute to a particular observed class. While the decay classes remain fixed, we are able to redefine the list of decay modes that we wish to measure as long as there remains a distinct signature for at least one of the sub-decays of each of the modes. Of course, each set of decay modes requires a separate calculation of the $T_{i j-k l}^{\tau \uparrow}$ elements.

\subsubsection{Estimation of Systematic Errors}

Before proceeding with the results of the branching fraction analysis, we consider the sources of systematic uncertainty in our measurements. From the mathematical formalism of our calculations, we expect that the dominant sources for systematic errors are in the estimation of the classification efficiency matrix elements $T_{i j-k l}^{+*}$, which include effects due to both the selection and classification processes. The error due to uncertainties in the integrated luminosity, which is estimated to be $63 \pm 7 \mathrm{pb}^{-1}$, is eliminated by normalization of the branching fractions to unity.

An overview of the estimation of systematic errors for the decay classification is given below. The systematic error contributions due to the various sources for the set of decay class branching fractions are listed in Table 5.11.

\section{Charged Particle Identification}

The major source of systematic uncertainty in the branching fractions for the leptonic decays, $\tau^{-} \rightarrow \nu_{\tau} \bar{\nu}_{e} e^{-}$and $\tau^{-} \rightarrow \nu_{\tau} \bar{\nu}_{\mu} \mu^{-}$, is the simulation of the charged 


\begin{tabular}{lccccc}
\hline Decay Class & \multicolumn{5}{c}{ Systematic Errors (\%) } \\
& 1 & 2 & 3 & 4 & total \\
\hline$e^{-}$ & 0.91 & 0.19 & 1.01 & 0.13 & 1.38 \\
$\mu^{-}$ & 1.05 & 0.09 & 0.90 & 0.14 & 1.39 \\
$\pi^{-}$ & 0.77 & 1.75 & 0.65 & 0.12 & 2.02 \\
$\pi^{-} \pi^{0}$ & 0.69 & 2.24 & 1.47 & 0.20 & 2.77 \\
$\pi^{-} \pi^{0} \pi^{0}$ & 0.76 & 3.61 & 0.91 & 0.05 & 3.80 \\
$\pi^{-} \pi^{0} \pi^{0} \pi^{0}$ & 1.01 & 2.12 & 0.30 & 0.05 & 2.37 \\
$K^{-}$ & 0.11 & 0.11 & 0.06 & 0.08 & 0.19 \\
$K^{-} \pi^{0}$ & 0.13 & 0.26 & 0.07 & 0.03 & 0.30 \\
$K^{-} \pi^{0} \pi^{0}$ & 0.25 & 0.45 & 0.05 & 0.03 & 0.52 \\
$\pi^{-} \pi^{+} \pi^{-}$ & 0.47 & 0.26 & 0.47 & 0.10 & 0.72 \\
$\pi^{-} \pi^{+} \pi^{-} \pi^{0}$ & 0.33 & 0.44 & 0.35 & 0.10 & 0.66 \\
$K^{-} \pi^{+} \pi^{-}$ & 0.25 & 0.03 & 0.03 & 0.05 & 0.26 \\
$K^{-} K^{+} \pi^{-}$ & 0.05 & 0.03 & 0.01 & 0.09 & 0.11 \\
\hline
\end{tabular}

Table 5.11: Systematic errors for decay class branching fractions. The individual contributions, 1 - charged particle identification, 2 - neutral particle reconstruction, 3 selection efficiency, 4 - background subtraction are added in quadrature to obtain the net systematic errors.

particle classification. In order to estimate the systematic error associated with this uncertainty we vary the parameterization of the $\mathrm{d} E / \mathrm{d} x$ resolution, and the (mis)identification probabilities for electrons, muons, and hadrons in the electromagnetic calorimeter and muon detection system.

\section{Neutral Particle Classification}

Compared with the identification of the charged tracks, the uncertainty in the classification of the neutrals in the $\tau^{-}$decays is relatively large. In order to estimate this uncertainty we use different definitions of the neutral classes. If we use the number of fully reconstructed $\pi^{0} \mathrm{~s}$ (excluding additional energetic photons) to define the various neutral particle classes, the branching fraction estimates tend to drop for the multiple $\pi^{0}$ modes, while the modes with no $\pi^{0} \mathrm{~s}, \tau^{-} \rightarrow \nu_{\tau} \pi^{-}$and $\tau^{-} \rightarrow \nu_{\tau} K^{-}$, show increases. This is an indication that the Monte Carlo may be overly optimistic in the simulation of $\pi^{0}$ reconstruction in $\tau^{-}$decays. At the other extreme, we may use the total number of energetic photons as the definition of the neutrals classes. In this case we find that the Monte Carlo underestimates the number of clusters in the calorimetry, and the branching fractions for modes with multiple $\pi^{0} \mathrm{~s}$ increase. 


\begin{tabular}{|c|c|c|c|}
\hline \multirow[t]{2}{*}{ Decay Class } & \multicolumn{3}{|c|}{ Branching Fractions \% } \\
\hline & TPC/Two-Gamma & World & verage \\
\hline$e^{-}$ & $17.25 \pm 0.95 \pm 1.38$ & $17.89 \pm 0.14$ & \\
\hline$\mu^{-}$ & $17.19 \pm 0.89 \pm 1.39$ & $17.34 \pm 0.16$ & \\
\hline$\pi^{-}$ & $9.43 \pm 0.79 \pm 2.02$ & $11.6 \pm 0.4$ & \\
\hline$\pi^{-} \pi^{0}$ & $26.73 \pm 1.29 \pm 2.77$ & $22.2 \pm 1.0$ & \\
\hline$\pi^{-} \pi^{0} \pi^{0}$ & $11.58 \pm 1.68 \pm 3.80$ & $(9.0 \pm 0.8$ & $\left.h^{-} \pi^{0} \pi^{0}\right)$ \\
\hline$\pi^{-} \pi^{0} \pi^{0} \pi^{0}$ & $1.32 \pm 1.11 \pm 2.37$ & $(1.8 \pm 0.5$ & $\left.h^{-} \pi^{0} \pi^{0} \pi^{0}\right)$ \\
\hline$K^{-}$ & $0.62 \pm 0.22 \pm 0.19$ & $0.67 \pm 0.23$ & \\
\hline$K^{-} \pi^{0}$ & $0.78 \pm 0.26 \pm 0.30$ & & \\
\hline$K^{-} \pi^{0} \pi^{0}$ & $0.42 \pm 0.22 \pm 0.52$ & & \\
\hline$\pi^{-} \pi^{+} \pi^{-}$ & $7.87 \pm 0.59 \pm 0.72$ & $(8.2 \pm 0.6$ & $\left.h^{-} h^{+} h^{-}\right)$ \\
\hline$\pi^{-} \pi^{+} \pi^{-} \pi^{0}$ & $6.29 \pm 0.54 \pm 0.66$ & $(4.8 \pm 0.6$ & $\left.h-h^{+} h^{-} \pi^{0}\right)$ \\
\hline$K^{-} \pi^{+} \pi^{-}$ & $0.33 \pm 0.19 \pm 0.26$ & $0.22 \pm 0.16$ & \\
\hline$K^{-} K^{+} \pi^{-}$ & $0.19 \pm 0.11 \pm 0.11$ & $0.22 \pm 0.17$ & \\
\hline
\end{tabular}

Table 5.12: $\tau^{-}$branching fractions for the decay classes of this analysis, compared with available world averages. The errors are statistical and systematic.

Varying the neutral classification in this way produces different sets of values for the the event class populations $n_{i j}$. If the simulation accurately describes the interaction of the decay particles with the detector, the values of $T_{i j-k l}^{\pi}$, calculated with the modified classification scheme, will compensate for the changes in the event class populations such that the branching fraction values remain stable. The branching fractions obtained with these two alternate neutral class definitions provide estimates of the systematic errors from neutral particle reconstruction.

\subsubsection{Decay Classes}

The most obvious set of branching fractions that we might measure is simply the set of decay classes that we have defined. This is certainly the most empirical since we report the decays based on what we actually observe in the detector, not on any theoretical assumptions about the resonances that the decays have passed through. However, this approach may combine physically distinct decays that happen to contribute to the same decay class. For example, we expect that the decays $\tau^{-} \rightarrow \nu_{\tau} \pi^{-}$and $\tau^{-} \rightarrow \nu_{\tau} K^{*-}$ will both contribute to the class $\pi^{-}$, since the $K^{*-}$ may decay to $\pi^{-} K_{L}^{0}$. The branching fraction reported here for $\pi^{-}$says nothing about the how much of the decay rate is from which source.

Using the class efficiency matrix elements for the dominant decay channels for 
each of the decay classes, we arrive at the branching fractions listed in Table 5.12. Our measurements of the leptonic modes are consistent with the current world average values, and provide a check of the overall consistency of our global set of branching fractions.

Our values for the $\pi^{-}$and $\pi^{-} \pi^{0}$ branching fractions are notably lower and higher, respectively, than the world averages, although the large systematic errors due to uncertainties in the neutral classification of decays limit the precision of our measurements. Our values for $\pi^{-} \pi^{0}$ and $\pi^{-} \pi^{0} \pi^{0}$ are the first measurements of these decay modes. We compare these with the world average values for $h^{-} \pi^{0}$ and $h^{-} \pi^{0} \pi^{0}$, where the $h^{-}$stands for hadron, either $\pi^{-}$or $K^{-}$. As for decays with one charged kaon, our value for $K^{-}$is in good agreement with the world average value, while our measurements for $K^{-} \pi^{0}$ and $K^{-} \pi^{0} \pi^{0}$ have no precedent.

The branching fractions for the three-prong decay classes $\pi^{-} \pi^{+} \pi^{-}$and $\pi^{-} \pi^{+} \pi^{-} \pi^{0}$, as with their one-prong analogs $\pi^{-} \pi^{0} \pi^{0}$ and $\pi^{-} \pi^{0} \pi^{0} \pi^{0}$, are first measurements. The precision of these three-prong decay measurements is much better than that of their one-prong analogs since the systematic error introduced by the uncertainties in the neutral classification is much lower. Our values for the three-prong decays with charged kaons, $K^{-} \pi^{+} \pi^{-}$and $K^{-} K^{+} \pi^{-}$, are consistent with the world average values.

\subsubsection{Resonance Classes}

Another approach to the definition of a set of decay modes is to assume that the hadronic decays of the $\tau^{-}$are dominated by resonances. Here we assume that the $(\pi \pi)^{-},(\pi \pi \pi)^{-},(K \pi)^{-}$, and $(K \pi \pi)^{-}$decays are dominated by the $\rho^{-}, a_{1}^{-}, K^{*-}$, and $K_{1}^{-}$channels respectively. The $\rho^{-}$decays to the final state $\pi^{-} \pi^{0}$, while the $a_{1}^{-}$ decays to a $1: 1$ ratio of $\pi^{-} \pi^{0} \pi^{0}$ and $\pi^{-} \pi^{+} \pi^{-}$.

As discussed earlier, the $\bar{u} s$ channels have more complicated decay patterns, and this analysis uses the decays that include charged $K$ as signatures for these decays. About one-third of the $\tau^{-} \rightarrow \nu_{\tau} K^{*-}$ decays result in the $K^{-} \pi^{0}$ final state, while about one-ninth of the $\tau^{-} \rightarrow \nu_{\tau} K_{1}^{-}$decays result in the $K^{-} \pi^{0} \pi^{0}$ final state and four-ninths result in the $K^{-} \pi^{+} \pi^{-}$final state. The remaining $K^{*-}$ and $K_{1}^{-}$decays lead to final states that are dominated by $\bar{u} d$ decay channels. By using a set of resonant channels for the $\tau^{-}$decays, we are able to subtract these backgrounds from the $\bar{u} d$ measurements.

As a check that the hadronic decays observed in our data sample exhibit this pattern of resonant state dominance, Figure 5.6 shows invariant mass plots for the charged and neutral decay particles of particular decay classcs, compared with Monte Carlo simulations of resonant channel decays. The upper plot includes decays in which there is one likely $\pi^{-}$, one reconstructed $\pi^{0}$, and no additional energetic photons. A prominent $\rho^{-}$peak is observed. The middle plot is for decays 

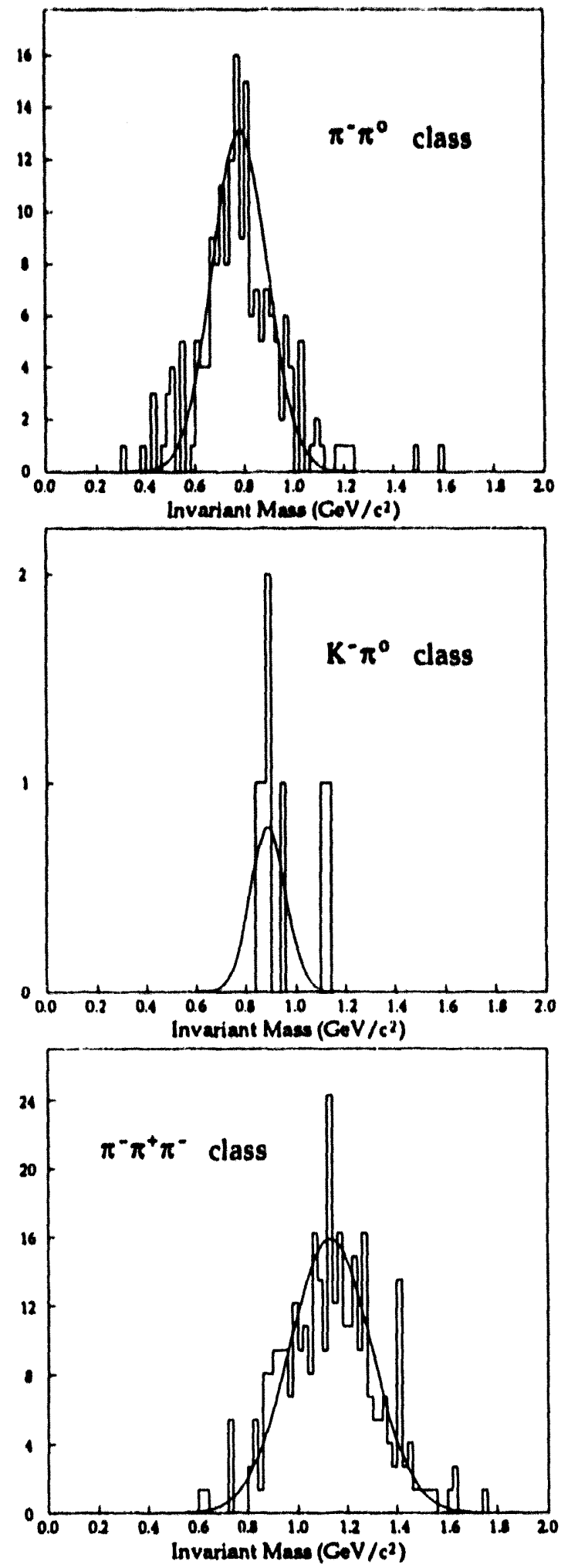

Figure 5.6: Mass distributions for $\pi^{-} \pi^{0}, K^{-} \pi^{0}, \pi^{-} \pi^{+} \pi^{-}$events, compared with Monte Carlo distributions for $\tau^{-} \rightarrow \nu_{\tau} \rho^{-} / \rho^{-} \rightarrow \pi^{-} \pi^{0}, \tau^{-} \rightarrow \nu_{\tau} K^{*-} / K^{*-} \rightarrow K^{-} \pi^{0}$, and $\tau^{-} \rightarrow \nu_{\tau} a_{1}^{-} / a_{1}^{-} \rightarrow \pi^{-} \pi^{+} \pi^{-}$events. 


\begin{tabular}{lrr}
\hline Decay Class & \multicolumn{2}{c}{ Branching Fractions \% } \\
& TPC/Two-Gamma & \multicolumn{1}{c}{ Theory } \\
\hline$e^{-}$ & $17.21 \pm 1.04 \pm 1.35$ & $18.13 \pm 0.20$ \\
$\mu^{-}$ & $17.11 \pm 0.96 \pm 1.40$ & $17.64 \pm 0.19$ \\
\hline$\pi^{-}$ & $9.44 \pm 0.85 \pm 2.07$ & $10.90 \pm 0.12$ \\
$K^{-}$ & $0.60 \pm 0.23 \pm 0.19$ & $0.73 \pm 0.01$ \\
\hline$\rho^{-}$ & $27.33 \pm 1.34 \pm 2.05$ \\
$K^{\circ-}$ & $2.89 \pm 0.80 \pm 0.81$ \\
\hline$a_{1}^{-}$ & $16.36 \pm 1.26 \pm 1.69$ \\
$K_{1}^{-}$ & $0.95 \pm 0.47 \pm 0.41$ \\
\hline$\pi^{-} \pi^{0} \pi^{0} \pi^{0}$ & $2.45 \pm 0.93 \pm 1.36$ \\
$\pi^{-} \pi^{+} \pi^{-} \pi^{0}$ & $5.41 \pm 0.64 \pm 0.47$ \\
$K^{-} K^{+} \pi^{-}$ & $0.19 \pm 0.11 \pm 0.14$ \\
\hline
\end{tabular}

Table 5.13: $\tau^{-}$branching fractions for resonance classes, assuming that the hadronic decays are dominated by these channels. The resonances are listed in $\bar{u} d$ and $\bar{u} s$ pairs.

with one likely $K^{-}$, one reconstructed $\pi^{0}$, and no additional photons. Although the statistics are small, the distribution is consistent with $K^{*-}$. Finally, the lower plot is for $\pi^{-} \pi^{+} \pi^{-}$candidate decays. Here the distribution is consistent with the $a_{1}^{-}$channel. Evidence for decays of the $\tau^{-}$through the $K_{1}^{-}$channels is presented in detail in the preceeding chapter.

The branching fraction results for the resonant-dominated set of decays are listed in Table 5.13. Note that the $\bar{u} s$ contributions to predominantly $\bar{u} d$ decay classes are removed automatically during the maximization of the likelihood function. For example, the $K^{*-}$ contribution is taken out of the $\pi^{-}$class by using the $K^{-} \pi^{0}$ class as a signature for the $K^{*-}$ decay. Another feature of this approach is that constraints are placed on the decays $\pi^{-} \pi^{0} \pi^{0}$ and $\pi^{-} \pi^{+} \pi^{-}$, since they are assumed to be produced at an equal rate through the $a_{1}^{-}$.

As expected, the modified set of hadronic decay modes has little effect on the leptonic branching fractions. The $\pi^{-}$and $K^{-}$values also show little change, even though the $\mathrm{K}^{*-}$ contribution to the $\pi^{-}$decay is now subtracted out. The measured branching fractions for these four simplest decays are all consistent with theory.

The measurements for the vector $\rho^{-}$and $K^{*-}$ decays are both higher than would be expected from our values for the $\pi^{-} \pi^{0}$ and $K^{-} \pi^{0}$ branching fractions. The value for $\rho^{-}$is somewhat higher than that for $\pi^{-} \pi^{0}$, in spite of the subtraction of $K_{1}^{-}$ contributions from the $\pi^{-} \pi^{0}$ decay class. Also, the $K^{*-}$ measurement is notably higher than three times that of the $K^{-} \pi^{0}$ decay. These vector channel values are higher than expected because of constraints imposed by the assumption of $a_{1}^{-}$ 
and $K_{1}^{-}$dominance. For the axial-vector $a_{1}^{-}$and $K_{1}^{-}$decays, the contributions from the three-prong final states $\left(\pi^{-} \pi^{+} \pi^{-}\right.$and $\left.K^{-} \pi^{+} \pi^{-}\right)$and the one-prong final states $\left(\pi^{-} \pi^{0} \pi^{0}\right.$ and $\left.K^{-} \pi^{0} \pi^{0}\right)$ are constrained to particular ratios, based on isospin symmetry. This limits the number of events that are attributed to $\pi^{-} \pi^{0} \pi^{0}$ and $K^{-} \pi^{0} \pi^{0}$ decays, and results in increased branching fractions for final states with 1 or $3 \pi^{0} \mathrm{~s}$. This effect is apparent in the higher than expected values for the $\rho^{-}$ and $K^{*-}$ decays, and in the $\pi^{-} \pi^{0} \pi^{0} \pi^{0}$ value, which is considerably higher for the resonant set of decays.

\subsection{Discussion}

This chapter has described the selection and classification scheme used to obtain a set of $\tau^{+} \tau^{-}$event samples. The populations of these event classes $n_{i j}$ are used along with classification efficiencies $T_{i j-k l}$ to determine branching fractions for a complete set of $\tau^{-}$decays.

The measurements for the leptonic decay modes are consistent with both the current world average values and the theoretical predicted values. Unfortunately, while the world average values for the leptonic branching fractions are at the $1 \%$ level, the size of our data set limits us to measurements at the $5 \%$ level. Nevertheless, our values for the leptonic modes are important in determining the overall consistency of our global set of measurements.

For all of the hadronic decay classes, our branching fraction measurements are either unique or have precision comparable to existing measurements. Although all of our measurements are consistent with existing world averages, our measurements for the vector decays $\tau^{-} \rightarrow \nu_{\tau} \rho^{-}$and $\tau^{-} \rightarrow \nu_{\tau} K^{*-}$ are considerably higher than existing measurements. Unfortunately, large systematic uncertainties in the neutral classification prevent us from making a more precise determination of these branching fractions. 


\section{Chapter 6}

\section{Conclusions}

This thesis describes measurements of branching fractions for the decays of the $\tau^{-}$ lepton. The analysis presented here is based on data from the TPC/Two-Gamma detector, which is capable of $\pi^{ \pm} / K^{ \pm}$particle identification. A particular focus of our analysis has been the measurement of branching fractions for decays with $K^{ \pm}$.

For the $K_{1}^{-}$decays of the $\tau^{-}$, we have obtained branching fraction measurements of,

$$
\begin{aligned}
& B\left[\tau^{-} \rightarrow \nu_{\tau} K_{1}^{-}(1270)\right]=0.41 \underbrace{+0.41 \%}_{-0.35} \% \\
& B\left[\tau^{-} \rightarrow \nu_{\tau} K_{1}^{-}(1400)\right]=0.76 \pm_{0.33}^{+0.40} \%
\end{aligned}
$$

The net branching fraction for the $K_{1}^{-}(1270)$ and $K_{1}^{-}(1400)$ decays is

$$
B\left[\tau^{-} \rightarrow \nu_{\tau} K_{1}^{-}\right]=1.17{ }_{-0.37}^{0.41} \%,
$$

consistent with the value expected from the $a_{1}^{-}$branching fraction and $\left|V_{u s}\right|^{2} /\left|V_{u d}\right|^{2}$ suppression.

This thesis also describes a global measurement of $\tau^{-}$lepton branching fractions, in which the $\tau^{-}$decays are classified according to the observed charged particles and reconstructed neutral particles. The $\pi^{ \pm} / K^{ \pm}$particle identification of the TPC allows us to make the first branching fraction measurements for a number of decay classes defined by the number of $\pi^{ \pm}, K^{ \pm}$, and $\pi^{0}$ in the decay. Assuming that the hadronic decays of the $\tau^{-}$lepton proceed through coupling of the $W^{-}$to resonant states, we obtain branching fraction measurements for the $\bar{u} d$ channels $\pi^{-}, \rho^{-}$, and $a_{1}^{-}$, and for the $\bar{u} s$ channels $K^{-}, K^{*-}$, and $K_{1}^{-}$.

While the $\pi^{ \pm} / K^{ \pm}$particle identification of the TPC/Two-Gamma detector has allowed us to obtain the first branching fraction measurements for a number of decay classes, the precision of these measurements has been limited statistically by the size of the data set and systematically by the uncertainties in the reconstruction of photons and $\pi^{0} \mathrm{~s}$. Improvements in the understanding of $\tau^{-}$lepton decay 
processes will require experiments that are capable of both charged particle identification and efficient neutral particle reconstruction, and that have large statistical samples. 


\section{Bibliography}

[1] K.G. Hayes, M.L. Perl, and B. Efron. Phys. Rev. D39, 274 (1989).

[2] K.G. Hayes and M.L. Perl. Phys. Rev. D38, 3351 (1988).

[3] H.J. Behrend et al. Phys. Lett. B 222, 163 (1989).

[4] D. Decamp et al. Z. Phys. C 54, 211 (1992).

[5] S.L. Glashow. Nucl. Phys. 22, 579 (1961).

[6] S. Weinberg. Phys. Rev. 112, 1375 (1958).

[7] A. Salam. In N. Svartholm, editor, Proceedings of the Eighth Nobel Symposium, page 367, New York, 1968. Wiley Interscience.

[8] M. Aguilar-Benitez et al. Review of Particle Properties. Phys. Rev. D45, 1 (1992).

[9] M.L. Perl et al. Phys. Rev. Lett. 35, 1489 (1975).

[10] A. Schwarz. Tau physics. In XVI International Symposium on Lepton-Photon Interactions, 1993.

[11] F. Halzen and A.D. Martin. Quarks and Leptons:An Introductory Course in Modern Particle Physics. John Wiley and Sons, New York, 1984.

[12] W.J. Marciano and A. Sirlin. Phys. Rev. Lett. 61, 1815 (1988).

[13] H. Marsiske. New measurements of the tau lepton mass. In K.K. Gan, editor, Second Workshop on Tau Lepton Physics, page 1, Columbus, Ohio, 1993.

[14] W. Trischuk. A review of the tau lifetime. In K.K. Gan, editor, Second Workshop on Tau Lepton Physics, page 59, Columbus, Ohio, 1993.

[15] James Jackson Eastman. Kaon Content of Three-Prong Decays of the Tau Lepton. PhD thesis, University of California, Berkeley, December 1990.

[16] H. Albrecht et al. Z. Phys. C41, 1 (1988). 
[17] W.B. Schmidke et al. Phys. Rev. Lett. 57, 527 (1986).

[18] H. Aihara et al. Phys. Rev. Lett. 59, 751 (1987).

[19] G.B. Mills et al. Phys. Rev. Lett. 54, 624 (1985).

[20] H. Aihara et al. Charged Hadron Production in $e^{+} e^{-}$Annihilation at $29 \mathrm{GeV}$ Center-of-Mass Energy. LBL Report LBL-23737, Lawrence Berkeley Laboratory, 1988.

[21] R. Fuzesy, N.J. Hadley, and P.R. Robrish. Nucl. Instr. Meth. 223, 40 (1984).

[22] Glen Douglas Cowan. Inclusive $\pi^{ \pm}, K^{ \pm}$, and $p, \bar{p}$ Production in $e^{+} e^{-} A n$ nihilation at $\sqrt{s}=29 \mathrm{GeV}$. PhD thesis, University of California, Berkeley, January 1988.

[23] H. Aihara et al. Nucl. Instr. Meth. 217, 259 (1983).

[24] R. I. Koda. A Test of Quantum Electrodynamics at Small Angles Using the PEP-4 Facility. PhD thesis, University of California, Los Angeles, 1985. UCLA-85-011.

[25] H. Aihara et al. IEEE Trans. Nucl. Sci. NS30, 67 (1983).

[26] Jon Alan Bakken. Prompt Muon Rate from $e^{+} e^{-}$Annihilations at $29 \mathrm{GeV}$. PhD thesis, Johns Hopkins University, 1983.

[27] M.T. Ronan. The PEP-4 (TPC) Trigger System. TPC Note TPC-LBL-87-12, Lawrence Berkeley Laboratory, 1987.

[28] William Walton Moses. Measurement of the Inclusive Branching Fraction $\tau^{-} \rightarrow \pi^{-} \pi^{0}+$ neutral meson(s). PhD thesis, University of California, Berkeley, December 1986.

[29] Y.S. Tsai. Phys. Rev. D4, 2821 (1971).

[30] M. Suzuki. Phys. Rev. D47, 1252 (1993).

[31] S. Jadach and Z. Was. Comp. Phys. Comm. 64, 275 (1991).

[32] M. Goldberg et al. Phys. Lett. 251B, 223 (1990).

[33] Wei-Ming Zhang. Measurement of Tau Branching Ratios. PhD thesis, Johns Hopkins University, 1986. 

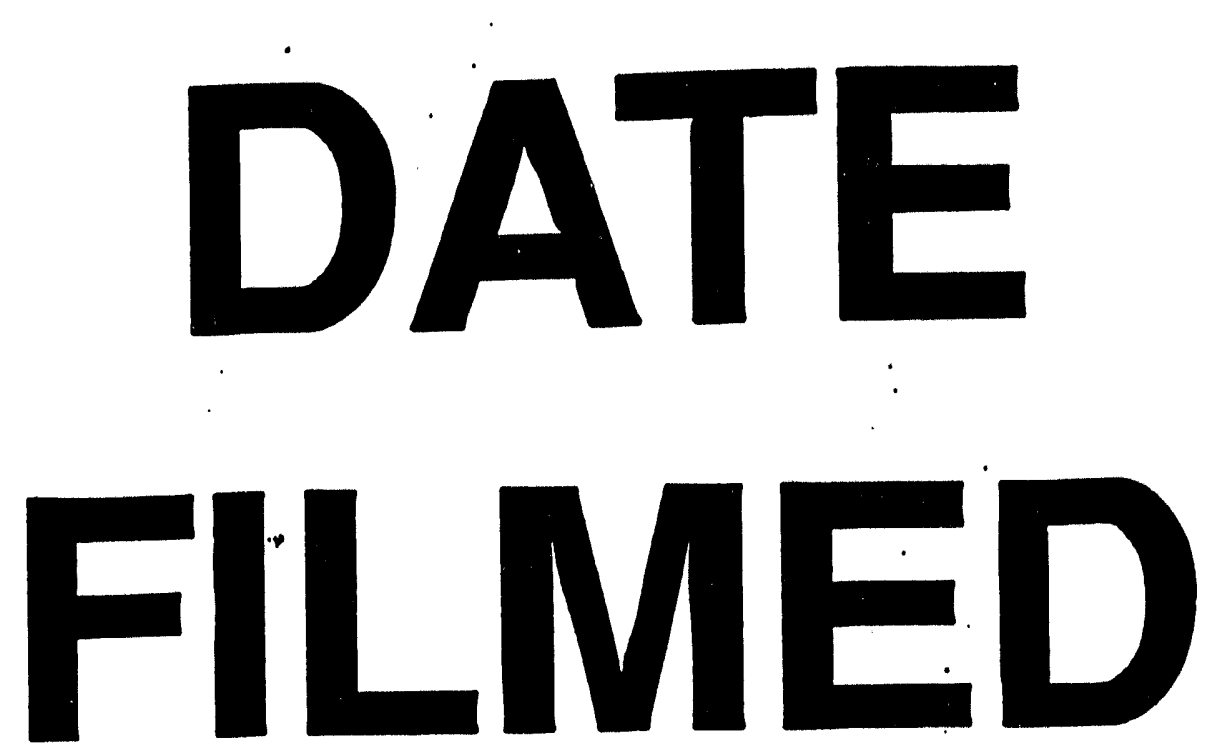

$1 / 5 / 94$
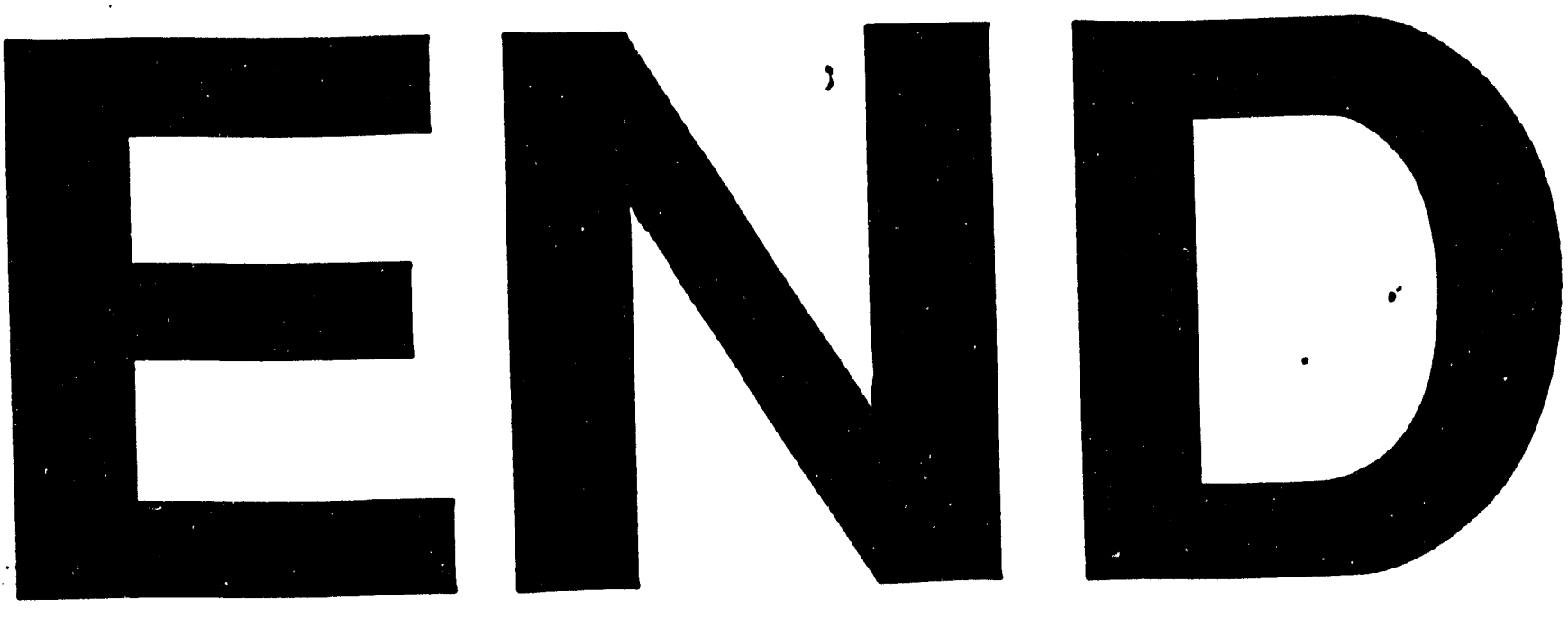


$$
\text { (1) }
$$

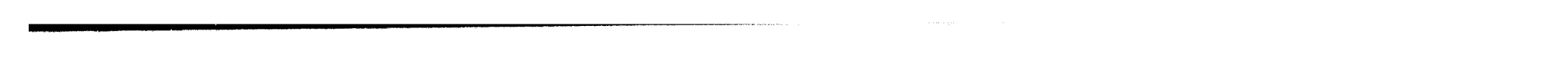

